\title{
Distinct Quality Changes of Garlic Bulb during Growth by Metabolomics Analysis
}

Pingxiang Liu, Rui Weng, Yanyang Xu, Yecan Pan, Beinan Wang, Yongzhong Qian*, Jing Qiu*

Institute of Quality Standard and Testing Technology for Agro-Products, Chinese Academy of Agricultural

Sciences, Key Laboratory of Agri-food Quality and Safety,

Ministry of Agriculture and Rural Affairs, Beijing, 100081, China

*Corresponding author. Tel.: +86 10-82106298, Fax: +86 10-82106517.

E-mail address: qyzcaas@ 163.com (Y. Qian)

qiujing@caas.cn (J. Qiu) 
Table S1 Identification of main chemical constituents in garlic by UHPLC Q-Exactive Orbitrap MS

Table S2 Detailed websites from MoNA that support the identification of 12 compounds in garlic

Table S3 Contents of 29 targeted compounds in garlic among different growing stages $(n=3)$

Table S4 Agro-morphological traits of garlic in different growing stages $(n=3)$

Table S5 Parameters of OPLS regression models for characteristic components in garlic based on agro-morphological traits

Table S6 The observed and predicted concentrations and relative error (RE) between them for testing set predicted by OPLS regression models

Table S7 The VIP values for agro-morphological traits in OPLS regression models for allicin and total 7 flavor precursors in ZP, $\mathrm{J} 3, \mathrm{~J} 4$, and $\mathrm{BP}$

Figure S1 Workflows and parameters of Compound Discovery 3.0 analysis

Figure S2 MS/MS spectrums of 29 compounds that identified in garlic sample (upper panel) and mzCloud (lower panel)

Figure S3 Structural characterization for 13 compounds in garlic

Figure S4 Figure S4 TIC obtained by UHPLC Q-Exactive Orbitrap MS of extracts from garlic bulbs of ZP (A), BP (B), J3

(C) and $\mathrm{J} 4$ (D) at week 1, week 4 (normal harvest time), and week 6 (positive ion mode)

Figure S5 Pathway analysis showing changing metabolism during garlic development. The size and color of the circle represented the pathway impact and pathway significance, respectively. The bigger and deeper circles at the upper-right corner were the pathways that had a strong impact with higher significance in garlic metabolism 
Table S1 Identification of main chemical constituents in garlic by UHPLC Q-Exactive Orbitrap MS

\begin{tabular}{|c|c|c|c|c|c|c|c|c|c|}
\hline Group & No. & Tentative identification & $\begin{array}{c}\text { Quantitative } \\
\text { analyzed }\end{array}$ & $\mathrm{RT}$ (min) & Formula & Adduct & $\begin{array}{c}\mathrm{m} / \mathrm{z} \\
\text { (Calculated) }\end{array}$ & $\begin{array}{c}\mathrm{m} / \mathrm{z} \\
\text { (Observed) }\end{array}$ & $\begin{array}{l}\text { Error } \\
(\mathrm{ppm})\end{array}$ \\
\hline \multirow{13}{*}{$\begin{array}{l}\text { Organosulfur } \\
\text { compounds }\end{array}$} & 1 & $\gamma$-L-Glutamyl-S-allyl-L-cysteine* & Yes & 8.72 & $\mathrm{C} 11 \mathrm{H} 18 \mathrm{~N} 2 \mathrm{O} 5 \mathrm{~S}$ & {$[\mathrm{M}+\mathrm{H}]^{+}$} & 291.1009 & 291.1004 & -1.9521 \\
\hline & 2 & $\gamma$-L-Glutamyl-S-methyl-L-cysteine* & Yes & 10.61 & C9H16N2O5S & {$[\mathrm{M}+\mathrm{H}]^{+}$} & 265.0853 & 265.0848 & -1.7685 \\
\hline & 3 & $\gamma$-L-Glutamyl-S-(trans-1-propenyl)- L-cysteine & I & 8.53 & $\mathrm{C} 11 \mathrm{H} 18 \mathrm{~N} 2 \mathrm{O} 5 \mathrm{~S}$ & {$[\mathrm{M}+\mathrm{H}]^{+}$} & 291.1009 & 291.1004 & -1.9521 \\
\hline & 4 & $\gamma$-Glutamyl-S-allylthio-L-cysteine & I & 7.92 & $\mathrm{C} 11 \mathrm{H} 18 \mathrm{~N} 2 \mathrm{O} 5 \mathrm{~S} 2$ & {$[\mathrm{M}+\mathrm{H}]^{+}$} & 323.0730 & 323.0723 & -2.1345 \\
\hline & 5 & $\begin{array}{l}\gamma \text {-Glutamyl-S-(1-propenyl)-L-cysteine } \\
\text { sulfoxide }\end{array}$ & l & 13.07 & $\mathrm{C} 11 \mathrm{H} 18 \mathrm{~N} 2 \mathrm{O} 6 \mathrm{~S}$ & {$[\mathrm{M}+\mathrm{H}]^{+}$} & 307.0958 & 307.0952 & -1.9189 \\
\hline & 6 & S-(trans-1-propenyl)-L-cysteine* & Yes & 4.67 & $\mathrm{C} 6 \mathrm{H} 11 \mathrm{NO} 2 \mathrm{~S}$ & {$[\mathrm{M}+\mathrm{H}]^{+}$} & 162.0583 & 162.0581 & -1.3939 \\
\hline & 7 & S-Allyl-L-cysteine* & Yes & 4.94 & C6H11NO2S & {$[\mathrm{M}+\mathrm{H}]^{+}$} & 162.0583 & 162.0581 & -1.4944 \\
\hline & 8 & S-Methyl-L-cysteine* & Yes & 6.92 & C4H9NO2S & {$[\mathrm{M}+\mathrm{H}]^{+}$} & 136.0427 & 136.0426 & -0.7549 \\
\hline & 9 & N-2-Propyn-1-ylcysteine & 1 & 7.79 & C6H9NO2S & {$[\mathrm{M}+\mathrm{H}]^{+}$} & 160.0427 & 160.0426 & -0.4736 \\
\hline & 10 & Alliin* & Yes & 7.81 & C6H11NO3S & {$[\mathrm{M}+\mathrm{H}]^{+}$} & 178.0532 & 178.0531 & -0.7049 \\
\hline & 11 & Methiin* & Yes & 10.16 & C4H9NO3S & {$[\mathrm{M}+\mathrm{H}]^{+}$} & 152.0376 & 152.0374 & -1.5644 \\
\hline & 12 & Cycloalliin & I & 9.28 & C6H11NO3S & {$[\mathrm{M}+\mathrm{H}]^{+}$} & 178.0532 & 178.0530 & -1.1144 \\
\hline & 13 & S-Propyl-L-cysteine sulfoxide & l & 7.54 & C6H13NO3S & {$[\mathrm{M}+\mathrm{H}]^{+}$} & 180.0689 & 180.0687 & -1.0579 \\
\hline \multirow{15}{*}{$\begin{array}{l}\text { Amino acids } \\
\text { and } \\
\text { derivatives }\end{array}$} & 14 & Arginine* & Yes & 14.39 & C6H14N4O2 & {$[\mathrm{M}+\mathrm{H}]^{+}$} & 175.1190 & 175.1187 & -1.4402 \\
\hline & 15 & Valine* $^{*}$ & Yes & 6.50 & $\mathrm{C} 5 \mathrm{H} 11 \mathrm{NO} 2$ & {$[\mathrm{M}+\mathrm{H}]^{+}$} & 118.0863 & 118.0865 & 2.0731 \\
\hline & 16 & Lysine* $^{*}$ & Yes & 14.83 & $\mathrm{C} 6 \mathrm{H} 14 \mathrm{~N} 2 \mathrm{O} 2$ & {$[\mathrm{M}+\mathrm{H}]^{+}$} & 147.1128 & 147.1126 & -1.3881 \\
\hline & 17 & Glutamine $^{*}$ & Yes & 10.75 & C5H10N2O3 & {$[\mathrm{M}+\mathrm{H}]^{+}$} & 147.0764 & 147.0762 & -1.4870 \\
\hline & 18 & Threonine* & Yes & 9.32 & $\mathrm{C} 4 \mathrm{H} 9 \mathrm{NO} 3$ & {$[\mathrm{M}+\mathrm{H}]^{+}$} & 120.0655 & 120.0656 & 0.6688 \\
\hline & 19 & Proline* & Yes & 6.71 & $\mathrm{C} 5 \mathrm{H} 9 \mathrm{NO} 2$ & {$[\mathrm{M}+\mathrm{H}]^{+}$} & 116.0706 & 116.0708 & 1.6792 \\
\hline & 20 & Tryptophan* & Yes & 5.46 & $\mathrm{C} 11 \mathrm{H} 12 \mathrm{~N} 2 \mathrm{O} 2$ & {$[\mathrm{M}-\mathrm{H}]^{-}$} & 203.0826 & 203.0818 & -3.9437 \\
\hline & 21 & Alanine* & Yes & 8.61 & $\mathrm{C} 3 \mathrm{H} 7 \mathrm{NO} 2$ & {$[\mathrm{M}+\mathrm{H}]^{+}$} & 90.0550 & 90.0554 & 4.8304 \\
\hline & 22 & Isoleucine $^{*}$ & Yes & 5.38 & $\mathrm{C} 6 \mathrm{H} 13 \mathrm{NO} 2$ & {$[\mathrm{M}+\mathrm{H}]^{+}$} & 132.1019 & 132.1018 & -0.7964 \\
\hline & 23 & Aspartic acid* & Yes & 11.16 & C4H7NO4 & {$[\mathrm{M}+\mathrm{H}]^{+}$} & 134.0448 & 134.0447 & -0.6281 \\
\hline & 24 & Histidine $^{*}$ & Yes & 14.16 & $\mathrm{C} 6 \mathrm{H} 9 \mathrm{~N} 3 \mathrm{O} 2$ & {$[\mathrm{M}+\mathrm{H}]^{+}$} & 156.0768 & 156.0768 & 0.3005 \\
\hline & 25 & Asparagine* & Yes & 11.05 & $\mathrm{C} 4 \mathrm{H} 8 \mathrm{~N} 2 \mathrm{O} 3$ & {$[\mathrm{M}+\mathrm{H}]^{+}$} & 133.0608 & 133.0607 & -0.5163 \\
\hline & 26 & Phenylalanine* & Yes & 5.15 & $\mathrm{C} 9 \mathrm{H} 11 \mathrm{NO} 2$ & {$[\mathrm{M}+\mathrm{H}]^{+}$} & 166.0863 & 166.0860 & -1.5366 \\
\hline & 27 & Glutamic acid* & Yes & 10.09 & C5H9NO4 & {$[\mathrm{M}+\mathrm{H}]^{+}$} & 148.0604 & 148.0602 & -1.5825 \\
\hline & 28 & Serine* & Yes & 10.74 & $\mathrm{C} 3 \mathrm{H} 7 \mathrm{NO} 3$ & {$[\mathrm{M}+\mathrm{H}]^{+}$} & 106.0499 & 106.0502 & 3.1155 \\
\hline
\end{tabular}




\begin{tabular}{|c|c|c|c|c|c|c|c|c|c|}
\hline & 29 & Leucine* & Yes & 5.08 & $\mathrm{C} 6 \mathrm{H} 13 \mathrm{NO} 2$ & {$[\mathrm{M}+\mathrm{H}]^{+}$} & 132.1019 & 132.1018 & -0.7964 \\
\hline & 30 & Methionine $^{*}$ & Yes & 6.07 & $\mathrm{C} 5 \mathrm{H} 11 \mathrm{NO} 2 \mathrm{~S}$ & {$[\mathrm{M}+\mathrm{H}]^{+}$} & 150.0583 & 150.0581 & -1.5054 \\
\hline & 31 & Tyrosine* & Yes & 7.16 & C9H11NO3 & {$[\mathrm{M}+\mathrm{H}]^{+}$} & 182.0812 & 182.0810 & -0.9326 \\
\hline & 32 & $\gamma$-Aminobutyrate acid* & Yes & 5.58 & $\mathrm{C} 4 \mathrm{H} 9 \mathrm{NO} 2$ & {$[\mathrm{M}+\mathrm{H}]^{+}$} & 104.0706 & 104.0708 & 1.8728 \\
\hline & 33 & 4-Guanidinobutyric acid ${ }^{\text {मु }}$ & I & 4.57 & $\mathrm{C} 5 \mathrm{H} 11 \mathrm{~N} 3 \mathrm{O} 2$ & {$[\mathrm{M}+\mathrm{H}]^{+}$} & 146.0924 & 146.0921 & -2.0754 \\
\hline & 34 & Ornithine $^{*}$ & I & 14.86 & $\mathrm{C} 5 \mathrm{H} 12 \mathrm{~N} 2 \mathrm{O} 2$ & {$[\mathrm{M}+\mathrm{H}]^{+}$} & 133.0972 & 133.0971 & -0.4072 \\
\hline & 35 & Pipecolic acid* & I & 7.09 & $\mathrm{C} 6 \mathrm{H} 11 \mathrm{NO} 2$ & {$[\mathrm{M}+\mathrm{H}]^{+}$} & 130.0863 & 130.0862 & -0.4243 \\
\hline & 36 & 5-Oxoproline * & I & 2.23 & $\mathrm{C} 5 \mathrm{H} 7 \mathrm{NO} 3$ & {$[\mathrm{M}+\mathrm{H}]^{+}$} & 130.0499 & 130.0498 & -0.5352 \\
\hline & 37 & Ergothioneine ${ }^{\text {it }}$ & I & 8.39 & $\mathrm{C} 9 \mathrm{H} 15 \mathrm{~N} 3 \mathrm{O} 2 \mathrm{~S}$ & {$[\mathrm{M}+\mathrm{H}]^{+}$} & 230.0958 & 230.0955 & -1.1908 \\
\hline & 38 & Citrulline $^{\star}$ & I & 11.76 & $\mathrm{C} 6 \mathrm{H} 13 \mathrm{~N} 3 \mathrm{O} 3$ & {$[\mathrm{M}+\mathrm{H}]^{+}$} & 176.1030 & 176.1028 & -0.9529 \\
\hline & 39 & Arginine methyl ester ${ }^{\star}$ & l & 11.03 & $\mathrm{C} 7 \mathrm{H} 16 \mathrm{~N} 4 \mathrm{O} 2$ & {$[\mathrm{M}+\mathrm{H}]^{+}$} & 189.1346 & 189.1343 & -1.5983 \\
\hline & 40 & S-(2-Carboxypropyl)cysteine & I & 7.43 & C7H13NO4S & {$[\mathrm{M}+\mathrm{H}]^{+}$} & 208.0638 & 208.0635 & -1.3466 \\
\hline & 41 & $\gamma$-Glutamylphenylalanine ${ }^{*}$ & l & 8.13 & $\mathrm{C} 14 \mathrm{H} 18 \mathrm{~N} 2 \mathrm{O} 5$ & {$[\mathrm{M}+\mathrm{H}]^{+}$} & 295.1288 & 295.1284 & -1.6755 \\
\hline & 42 & Glutathion* $^{*}$ & / & 11.27 & $\mathrm{C} 10 \mathrm{H} 17 \mathrm{~N} 3 \mathrm{O} 6 \mathrm{~S}$ & {$[\mathrm{M}+\mathrm{H}]^{+}$} & 308.0911 & 308.0905 & -1.8092 \\
\hline & 43 & N6,N6,N6-Trimethyl-L-lysine & I & 13.54 & $\mathrm{C} 9 \mathrm{H} 20 \mathrm{~N} 2 \mathrm{O} 2$ & {$[\mathrm{M}+\mathrm{H}]^{+}$} & 189.1598 & 189.1593 & -2.2513 \\
\hline & 44 & L- $\gamma$-Glutamyl-L-leucine ${ }^{*}$ & I & 7.76 & $\mathrm{C} 11 \mathrm{H} 20 \mathrm{~N} 2 \mathrm{O} 5$ & {$[\mathrm{M}+\mathrm{H}]^{+}$} & 261.1445 & 261.1440 & -1.9077 \\
\hline & 45 & $\gamma$-Glutamyltyrosine ${ }^{\star}$ & I & 9.75 & $\mathrm{C} 14 \mathrm{H} 18 \mathrm{~N} 2 \mathrm{O} 6$ & {$[\mathrm{M}+\mathrm{H}]^{+}$} & 311.1238 & 311.1231 & -2.1303 \\
\hline & 46 & $\gamma$-Glutamyl-L-valine ${ }^{\star}$ & I & 8.68 & $\mathrm{C} 10 \mathrm{H} 18 \mathrm{~N} 2 \mathrm{O} 5$ & {$[\mathrm{M}+\mathrm{H}]^{+}$} & 247.1288 & 247.1285 & -1.3355 \\
\hline & 47 & N3,N4-Dimethyl-L-arginine ${ }^{\text {h }}$ & l & 12.56 & $\mathrm{C} 8 \mathrm{H} 18 \mathrm{~N} 4 \mathrm{O} 2$ & {$[\mathrm{M}+\mathrm{H}]^{+}$} & 203.1503 & 203.1502 & -0.2579 \\
\hline & 48 & N6-Acetyl-L-lysine ${ }^{\text {ht }}$ & I & 9.44 & $\mathrm{C} 8 \mathrm{H} 16 \mathrm{~N} 2 \mathrm{O} 3$ & {$[\mathrm{M}+\mathrm{H}]^{+}$} & 189.1234 & 189.1231 & -1.4218 \\
\hline & 49 & L-Saccharopine $\mathrm{H}^{\text {h }}$ & I & 15.61 & $\mathrm{C} 11 \mathrm{H} 20 \mathrm{~N} 2 \mathrm{O} 6$ & {$[\mathrm{M}+\mathrm{H}]^{+}$} & 277.1394 & 277.1390 & -1.4895 \\
\hline & 50 & Argininosuccinic acid & I & 16.09 & $\mathrm{C} 10 \mathrm{H} 18 \mathrm{~N} 4 \mathrm{O} 6$ & {$[\mathrm{M}+\mathrm{H}]^{+}$} & 291.1299 & 291.1291 & -2.7850 \\
\hline & 51 & $\gamma$-Glutamylcysteine ${ }^{\text {iz }}$ & 1 & 8.49 & C8H14N2O5S & {$[\mathrm{M}+\mathrm{H}]^{+}$} & 251.0696 & 251.0692 & -1.6677 \\
\hline & 52 & 1-Methylhistidine ${ }^{\text {iz }}$ & I & 5.90 & $\mathrm{C} 7 \mathrm{H} 11 \mathrm{~N} 3 \mathrm{O} 2$ & {$[\mathrm{M}+\mathrm{H}]^{+}$} & 170.0924 & 170.0922 & -1.1946 \\
\hline & 53 & $\gamma$-glutamylmethionine ${ }^{\star}$ & I & 9.09 & $\mathrm{C} 10 \mathrm{H} 18 \mathrm{~N} 2 \mathrm{O} 5 \mathrm{~S}$ & {$[\mathrm{M}+\mathrm{H}]^{+}$} & 279.1009 & 279.1005 & -1.3236 \\
\hline & 54 & Tilarginine $^{\star}$ & I & 13.21 & $\mathrm{C} 7 \mathrm{H} 16 \mathrm{~N} 4 \mathrm{O} 2$ & {$[\mathrm{M}+\mathrm{H}]^{+}$} & 189.1346 & 189.1344 & -1.2309 \\
\hline & 55 & N(6)-Methyllysine & I & 13.68 & $\mathrm{C} 7 \mathrm{H} 16 \mathrm{~N} 2 \mathrm{O} 2$ & {$[\mathrm{M}+\mathrm{H}]^{+}$} & 161.1285 & 161.1283 & -1.0069 \\
\hline & 56 & $\mathrm{~N}(6), \mathrm{N}(6)$-Dimethyl-L-lysine & I & 13.23 & $\mathrm{C} 8 \mathrm{H} 18 \mathrm{~N} 2 \mathrm{O} 2$ & {$[\mathrm{M}+\mathrm{H}]^{+}$} & 175.1441 & 175.1439 & -1.1182 \\
\hline & 57 & N-Methyl-L-methionine & 1 & 4.53 & C6H13NO2S & {$[\mathrm{M}+\mathrm{H}]^{+}$} & 164.0740 & 164.0737 & -1.6816 \\
\hline & 58 & $\delta$-Guanidinovaleric acid & I & 3.89 & $\mathrm{C} 6 \mathrm{H} 13 \mathrm{~N} 3 \mathrm{O} 2$ & {$[\mathrm{M}+\mathrm{H}]^{+}$} & 160.1081 & 160.1079 & -0.9569 \\
\hline Nucleotides & 59 & S-Adenosylmethionine $e^{\mu}$ & I & 15.66 & $\mathrm{C} 15 \mathrm{H} 22 \mathrm{~N} 6 \mathrm{O} 5 \mathrm{~S}$ & {$[\mathrm{M}+\mathrm{H}]^{+}$} & 399.1445 & 399.1441 & -1.1444 \\
\hline and & 60 & Adenosine $\mathrm{e}^{\text {मे }}$ & I & 2.77 & $\mathrm{C} 10 \mathrm{H} 13 \mathrm{~N} 5 \mathrm{O} 4$ & {$[\mathrm{M}+\mathrm{H}]^{+}$} & 268.1040 & 268.1035 & -1.9783 \\
\hline
\end{tabular}




\begin{tabular}{|c|c|c|c|c|c|c|c|c|c|}
\hline \multirow[t]{12}{*}{ derivatives } & 61 & 2'-Deoxyadenosine ${ }^{\text {th }}$ & I & 2.30 & $\mathrm{C} 10 \mathrm{H} 13 \mathrm{~N} 5 \mathrm{O} 3$ & {$[\mathrm{M}+\mathrm{H}]^{+}$} & 252.1091 & 252.1088 & -1.2526 \\
\hline & 62 & 3-Methyladenine $e^{\text {ir }}$ & 1 & 4.41 & C6H7N5 & {$[\mathrm{M}+\mathrm{H}]^{+}$} & 150.0774 & 150.0773 & -0.8116 \\
\hline & 63 & Adenine $^{\text {ht }}$ & I & 2.54 & C5H5N5 & {$[\mathrm{M}+\mathrm{H}]^{+}$} & 136.0618 & 136.0617 & -0.5277 \\
\hline & 64 & Uridine $^{\text {th }}$ & I & 2.88 & C9H12N2O6 & {$[\mathrm{M}-\mathrm{H}]^{-}$} & 243.0623 & 243.0616 & -2.5520 \\
\hline & 65 & Guanosine मे $^{2}$ & 1 & 5.85 & $\mathrm{C} 10 \mathrm{H} 13 \mathrm{~N} 5 \mathrm{O} 5$ & {$[\mathrm{M}-\mathrm{H}]^{-}$} & 282.0844 & 282.0842 & -0.6799 \\
\hline & 66 & 5'-S-Methyl-5'-thioadenosine ${ }^{\text {h }}$ & I & 1.62 & C11H15N5O3S & {$[\mathrm{M}+\mathrm{H}]^{+}$} & 298.0968 & 298.0963 & -1.8001 \\
\hline & 67 & Uridine $5^{\prime}$-diphosphate ${ }^{\star}$ & 1 & 17.20 & $\mathrm{C} 9 \mathrm{H} 14 \mathrm{~N} 2 \mathrm{O} 12 \mathrm{P} 2$ & {$[\mathrm{M}+\mathrm{H}]^{+}$} & 405.0095 & 405.0091 & -0.9215 \\
\hline & 68 & Uridine 5'-diphosphogalactose ${ }^{\text {h }}$ & I & 17.21 & $\mathrm{C} 15 \mathrm{H} 24 \mathrm{~N} 2 \mathrm{O} 17 \mathrm{P} 2$ & {$[\mathrm{M}-\mathrm{H}]^{-}$} & 565.0477 & 565.0475 & -0.4101 \\
\hline & 69 & 1-Methyladenosine ${ }^{\star}$ & I & 6.79 & $\mathrm{C} 11 \mathrm{H} 15 \mathrm{~N} 5 \mathrm{O} 4$ & {$[\mathrm{M}+\mathrm{H}]^{+}$} & 282.1197 & 282.1195 & -0.6398 \\
\hline & 70 & Pseudouridine & 1 & 4.71 & C9H12N2O6 & {$[\mathrm{M}-\mathrm{H}]^{-}$} & 243.0623 & 243.0617 & -2.3011 \\
\hline & 71 & 7-Methylguanosine & 1 & 7.44 & $\mathrm{C} 11 \mathrm{H} 15 \mathrm{~N} 5 \mathrm{O} 5$ & {$[\mathrm{M}+\mathrm{H}]^{+}$} & 298.1146 & 298.1142 & -1.3253 \\
\hline & 72 & Cytosine & 1 & 4.77 & C4H5N3O & {$[\mathrm{M}+\mathrm{H}]^{+}$} & 112.0505 & 112.0507 & 1.4422 \\
\hline \multirow{6}{*}{$\begin{array}{l}\text { Alkaloids } \\
\text { and } \\
\text { derivatives }\end{array}$} & 73 & Betaine $^{*}$ & 1 & 5.58 & $\mathrm{C} 5 \mathrm{H} 11 \mathrm{NO} 2$ & {$[\mathrm{M}+\mathrm{H}]^{+}$} & 118.0863 & 118.0863 & 0.3794 \\
\hline & 74 & Trigonelline* & I & 5.99 & $\mathrm{C} 7 \mathrm{H} 7 \mathrm{NO} 2$ & {$[\mathrm{M}+\mathrm{H}]^{+}$} & 138.0550 & 138.0543 & -4.7445 \\
\hline & 75 & Choline $^{*}$ & I & 3.22 & C5H13NO & {$[\mathrm{M}+\mathrm{H}]^{+}$} & 104.1070 & 104.1072 & 2.0114 \\
\hline & 76 & Acetylcholine & 1 & 3.67 & $\mathrm{C} 7 \mathrm{H} 15 \mathrm{NO} 2$ & {$[\mathrm{M}+\mathrm{H}]^{+}$} & 146.1176 & 146.1172 & -2.4316 \\
\hline & 77 & Choline sulfate & I & 2.29 & C5H13NO4S & {$[\mathrm{M}+\mathrm{H}]^{+}$} & 184.0638 & 184.0631 & -3.6134 \\
\hline & 78 & Stachydrine $e^{\text {मr }}$ & 1 & 5.29 & $\mathrm{C} 7 \mathrm{H} 13 \mathrm{NO} 2$ & {$[\mathrm{M}+\mathrm{H}]^{+}$} & 144.1019 & 144.1014 & -3.4365 \\
\hline \multirow{5}{*}{$\begin{array}{l}\text { Saccharides } \\
\text { and } \\
\text { derivatives }\end{array}$} & 79 & Sucrose $^{*}$ & 1 & 10.25 & $\mathrm{C} 12 \mathrm{H} 22 \mathrm{O} 11$ & {$[\mathrm{M}+\mathrm{FA}-\mathrm{H}]^{-}$} & 387.1144 & 387.1139 & -1.3174 \\
\hline & 80 & D-Raffinose $e^{\text {ht }}$ & 1 & 13.18 & $\mathrm{C} 18 \mathrm{H} 32 \mathrm{O} 16$ & {$[\mathrm{M}+\mathrm{Na}]^{+}$} & 527.1583 & 527.1583 & 0.0837 \\
\hline & 81 & Melezitose $^{\star}$ & 1 & 13.70 & $\mathrm{C} 18 \mathrm{H} 32 \mathrm{O} 16$ & {$[\mathrm{M}+\mathrm{NH} 4]^{+}$} & 522.2029 & 522.2029 & 0.0760 \\
\hline & 82 & Dulcitol $^{\text {मh }}$ & I & 6.31 & C6H14O6 & {$[\mathrm{M}-\mathrm{H}]^{-}$} & 181.0718 & 181.0710 & -4.2050 \\
\hline & 83 & Mannose 1-phosphate & 1 & 15.16 & C6H13O9P & {$[\mathrm{M}-\mathrm{H}]^{-}$} & 259.0224 & 259.0221 & -1.2444 \\
\hline \multirow{8}{*}{ Others } & 84 & Nicotinamide & 1 & 6.94 & C6H6N2O & {$[\mathrm{M}+\mathrm{H}]^{+}$} & 123.0553 & 123.0553 & 0.0861 \\
\hline & 85 & Pantothenic acid ${ }^{\text {मे }}$ & 1 & 1.39 & C9H17NO5 & {$[\mathrm{M}-\mathrm{H}]^{-}$} & 218.1034 & 218.1027 & -3.1907 \\
\hline & 86 & Pyridoxine ${ }^{\text {म }}$ & 1 & 2.51 & $\mathrm{C} 8 \mathrm{H} 11 \mathrm{NO} 3$ & {$[\mathrm{M}+\mathrm{H}]^{+}$} & 170.0812 & 170.0810 & -0.9983 \\
\hline & 87 & Phytosphingosine ${ }^{\text {hy }}$ & 1 & 2.43 & C18H39NO3 & {$[\mathrm{M}+\mathrm{H}]^{+}$} & 318.3003 & 318.2996 & -1.9588 \\
\hline & 88 & Choline Glycerophosphate & I & 11.68 & $\mathrm{C} 8 \mathrm{H} 20 \mathrm{NO} 6 \mathrm{P}$ & {$[\mathrm{M}+\mathrm{H}]^{+}$} & 258.1101 & 258.1097 & -1.5509 \\
\hline & 89 & Phenylacetaldehyde ${ }^{\star}$ & 1 & 3.07 & $\mathrm{C} 8 \mathrm{H} 8 \mathrm{O}$ & {$[\mathrm{M}+\mathrm{H}]^{+}$} & 121.0648 & 121.0648 & 0.0702 \\
\hline & 90 & 4-Guanidinobutanal & 1 & 3.45 & $\mathrm{C} 5 \mathrm{H} 11 \mathrm{~N} 3 \mathrm{O}$ & {$[\mathrm{M}+\mathrm{H}]^{+}$} & 130.0975 & 130.0975 & 0.0876 \\
\hline & 91 & 3-Methoxytyramine ${ }^{\star}$ & I & 2.82 & $\mathrm{C} 9 \mathrm{H} 10 \mathrm{O} 2$ & {$[\mathrm{M}+\mathrm{H}]^{+}$} & 151.0754 & 151.0747 & -4.2105 \\
\hline
\end{tabular}

Notes: * Compounds that confirmed by standards; $\underset{\sim}{ }$ Compounds that confirmed by mzCloud Database; $\star$ Compounds that confirmed by MoNADatabase 
Table S2 Detailed websites from MoNA that support the identification of 12 compounds in garlic

\begin{tabular}{|c|c|c|}
\hline No. & Compounds & Link of MoNA \\
\hline 38 & Citrulline & https://mona.fiehnlab.ucdavis.edu/spectra/display/FiehnHILIC000263 \\
\hline 39 & Arginine methyl ester & https://mona.fiehnlab.ucdavis.edu/spectra/display/MoNA011430 \\
\hline 45 & $\gamma$-Glutamyltyrosine & https://mona.fiehnlab.ucdavis.edu/spectra/display/FiehnHILIC000378 \\
\hline 46 & $\gamma$-Glutamyl-L-valine & https://mona.fiehnlab.ucdavis.edu/spectra/display/PM017302 \\
\hline 53 & $\gamma$-glutamylmethionine & https://mona.fiehnlab.ucdavis.edu/spectra/display/FiehnHILIC000377 \\
\hline 54 & Tilarginine & https://mona.fiehnlab.ucdavis.edu/spectra/display/FiehnHILIC000778 \\
\hline 67 & Uridine 5'-diphosphate & https://mona.fiehnlab.ucdavis.edu/spectra/display/CCMSLIB00000479751 \\
\hline 69 & 1-Methyladenosine & https://mona.fiehnlab.ucdavis.edu/spectra/display/FiehnHILIC000012 \\
\hline 81 & Melezitose & https://mona.fiehnlab.ucdavis.edu/spectra/display/VF-NPL-QEHF015037 \\
\hline 83 & $\alpha$-D-Mannose 1-phosphate & https://mona.fiehnlab.ucdavis.edu/spectra/display/KO001458 \\
\hline 89 & Phenylacetaldehyde & https://mona.fiehnlab.ucdavis.edu/spectra/display/FiehnHILIC000673 \\
\hline 91 & 3-Methoxytyramine & https://mona.fiehnlab.ucdavis.edu/spectra/display/CCMSLIB00000578072 \\
\hline
\end{tabular}


Table S3 Contents of 29 targeted compounds in garlic among different growing stages $(n=3)$

\begin{tabular}{|c|c|c|c|c|c|c|c|}
\hline compounds & variety & week 1 & week 2 & week 3 & week 4 & week 5 & week 6 \\
\hline \multirow{4}{*}{ SMC } & $\mathrm{ZP}$ & $0.7 \pm 0.2$ & $0.6 \pm 0.1$ & $0.7 \pm 0.2$ & $1.5 \pm 0.2$ & $2.4 \pm 0.1$ & $2.7 \pm 0.2$ \\
\hline & $\mathrm{J} 3$ & $36.7 \pm 1.0$ & $13.7 \pm 0.8$ & $4.9 \pm 0.9$ & $2.4 \pm 0.0$ & $1.4 \pm 0.1$ & $1.2 \pm 0.1$ \\
\hline & $\mathrm{J} 4$ & $14.3 \pm 2.6$ & $6.4 \pm 0.4$ & $3.5 \pm 1.2$ & $2.5 \pm 0.4$ & $1.8 \pm 0.7$ & $1.7 \pm 0.5$ \\
\hline & $\mathrm{BP}$ & $30.0 \pm 2.8$ & $9.2 \pm 1.6$ & $2.7 \pm 0.1$ & $2.5 \pm 0.6$ & $1.9 \pm 0.2$ & $1.3 \pm 0.2$ \\
\hline \multirow{4}{*}{ SAC } & $\mathrm{ZP}$ & $4.6 \pm 1.3$ & $2.8 \pm 0.5$ & $1.9 \pm 0.3$ & $3.2 \pm 0.2$ & $2.6 \pm 0.0$ & $3.2 \pm 0.5$ \\
\hline & $\mathrm{J} 3$ & $95.0 \pm 6.2$ & $34.9 \pm 1.5$ & $7.5 \pm 1.1$ & $2.5 \pm 0.2$ & $2.8 \pm 0.3$ & $2.7 \pm 0.1$ \\
\hline & $\mathrm{J} 4$ & $47.8 \pm 8.4$ & $20.0 \pm 1.1$ & $6.4 \pm 1.5$ & $2.7 \pm 0.3$ & $2.8 \pm 0.5$ & $2.2 \pm 0.1$ \\
\hline & $\mathrm{BP}$ & $85.4 \pm 11.7$ & $31.0 \pm 1.3$ & $5.7 \pm 0.4$ & $2.5 \pm 0.3$ & $2.4 \pm 0.4$ & $2.2 \pm 0.2$ \\
\hline \multirow{4}{*}{ SPC } & $\mathrm{ZP}$ & $2.6 \pm 0.9$ & $5.1 \pm 1.0$ & $2.0 \pm 0.2$ & $4.4 \pm 0.3$ & $4.6 \pm 1.6$ & $5.0 \pm 0.3$ \\
\hline & $\mathrm{J} 3$ & $96.6 \pm 11.8$ & $54.7 \pm 8$ & $6.9 \pm 1.8$ & $2.6 \pm 0.2$ & $2.1 \pm 0.2$ & $3.1 \pm 0.0$ \\
\hline & $\mathrm{J} 4$ & $44.0 \pm 7.8$ & $23.5 \pm 1.3$ & $5.5 \pm 1.0$ & $2.4 \pm 0.1$ & $1.9 \pm 0.4$ & $1.9 \pm 0.3$ \\
\hline & $\mathrm{BP}$ & $65.1 \pm 15.6$ & $41.0 \pm 3.0$ & $5.3 \pm 1.0$ & $2.2 \pm 0.3$ & $2.2 \pm 0.3$ & $2.3 \pm 0.1$ \\
\hline \multirow{4}{*}{ GSMC } & $\mathrm{ZP}$ & $34.0 \pm 4.0$ & $66.7 \pm 9.1$ & $132.4 \pm 11.9$ & $165.6 \pm 0.9$ & $271.9 \pm 16.7$ & $312.3 \pm 18.5$ \\
\hline & $\mathrm{J} 3$ & $73.1 \pm 25.7$ & $46.6 \pm 4.7$ & $142.9 \pm 41.4$ & $316.1 \pm 12.7$ & $210.6 \pm 25.8$ & $297.8 \pm 10.5$ \\
\hline & $\mathrm{J} 4$ & $53.4 \pm 10.1$ & $52.6 \pm 9.6$ & $97.9 \pm 22.1$ & $277.5 \pm 46.1$ & $276.6 \pm 76.2$ & $357.3 \pm 67$ \\
\hline & $\mathrm{BP}$ & $83.7 \pm 20.6$ & $54.0 \pm 7.3$ & $101 \pm 17.7$ & $290.3 \pm 31.7$ & $273.6 \pm 44.6$ & $450.2 \pm 56$ \\
\hline \multirow{4}{*}{ GSAC } & $\mathrm{ZP}$ & $322.7 \pm 18.4$ & $501.1 \pm 22.8$ & $755.1 \pm 15.2$ & $884.3 \pm 63.7$ & $979.7 \pm 70.6$ & $1073.0 \pm 107.5$ \\
\hline & $\mathrm{J} 3$ & $113.9 \pm 16.1$ & $102.7 \pm 4.2$ & $277.6 \pm 63.6$ & $489.2 \pm 23.8$ & $417.8 \pm 35.2$ & $531.2 \pm 7.3$ \\
\hline & $\mathrm{J} 4$ & $97.0 \pm 11.9$ & $120.3 \pm 6.8$ & $246 \pm 41.8$ & $381.6 \pm 42.1$ & $468.3 \pm 75.4$ & $540.4 \pm 23.9$ \\
\hline & $\mathrm{BP}$ & $157.7 \pm 37$ & $145.9 \pm 9.5$ & $274.5 \pm 39.5$ & $507.6 \pm 69.5$ & $444.6 \pm 47.2$ & $650.5 \pm 37.4$ \\
\hline \multirow{4}{*}{ Methiin } & $\mathrm{ZP}$ & $349.2 \pm 45.0$ & $514.7 \pm 19.1$ & $635.0 \pm 75.5$ & $664.1 \pm 21.8$ & $990.5 \pm 137.6$ & $1140.7 \pm 150.5$ \\
\hline & $\mathrm{J} 3$ & $1857.3 \pm 180.5$ & $1191.7 \pm 58.8$ & $1259.0 \pm 259.9$ & $1500.9 \pm 78.1$ & $1492.9 \pm 93.9$ & $1569.9 \pm 46.3$ \\
\hline & $\mathrm{J} 4$ & $1319.4 \pm 41.8$ & $1199.8 \pm 147.5$ & $1098.8 \pm 153.3$ & $1594.0 \pm 141.1$ & $1334.6 \pm 354.6$ & $1169.9 \pm 83.6$ \\
\hline & $\mathrm{BP}$ & $1500.3 \pm 45.1$ & $963.0 \pm 77.4$ & $907.2 \pm 69.2$ & $1203.2 \pm 251.9$ & $1285.9 \pm 96.5$ & $1369.3 \pm 100.9$ \\
\hline \multirow{4}{*}{ Alliin } & $\mathrm{ZP}$ & $619.9 \pm 115.2$ & $767.6 \pm 81.5$ & $904.8 \pm 251.9$ & $1417.9 \pm 142.9$ & $1731.6 \pm 42.0$ & $2055.4 \pm 385.6$ \\
\hline & $\mathrm{J} 3$ & $1797.6 \pm 248.4$ & $1175.8 \pm 67.4$ & $1224.5 \pm 235.0$ & $1742.7 \pm 39.4$ & $1934.9 \pm 67.2$ & $2310.5 \pm 207.6$ \\
\hline & $\mathrm{J} 4$ & $1501.3 \pm 86.1$ & $1579.9 \pm 323.3$ & $1363.9 \pm 136.2$ & $2022.8 \pm 332.8$ & $2050.6 \pm 410.4$ & $2047.6 \pm 340.0$ \\
\hline & $\mathrm{BP}$ & $1418.3 \pm 65.8$ & $1271.3 \pm 113$ & $1246.9 \pm 150.7$ & $1524.5 \pm 330.1$ & $1800.4 \pm 89.6$ & $2151.1 \pm 287.8$ \\
\hline \multirow{4}{*}{ Allicin } & $\mathrm{ZP}$ & $427.4 \pm 40.7$ & $463.9 \pm 29.7$ & $575.9 \pm 21.3$ & $741.3 \pm 36.6$ & $958.5 \pm 63.4$ & $1016.6 \pm 59.3$ \\
\hline & $\mathrm{J} 3$ & $609.9 \pm 66.5$ & $681.7 \pm 11.7$ & $771.2 \pm 73.3$ & $939.9 \pm 34.7$ & $1016.1 \pm 29.8$ & $1261.2 \pm 41.7$ \\
\hline & $\mathrm{J} 4$ & $675.6 \pm 31.6$ & $757.9 \pm 12.2$ & $723.7 \pm 52.9$ & $1034.5 \pm 30.9$ & $1136.9 \pm 63.7$ & $1338.0 \pm 19.2$ \\
\hline & $\mathrm{BP}$ & $724.3 \pm 36.8$ & $808.0 \pm 29.3$ & $784.0 \pm 17.8$ & $989.3 \pm 57.6$ & $1073.9 \pm 13.2$ & $1409.6 \pm 61.2$ \\
\hline \multirow{4}{*}{ Gly } & $\mathrm{ZP}$ & $1.1 \pm 0.1$ & $1.4 \pm 0.1$ & $1.4 \pm 0.2$ & $2.2 \pm 0.2$ & $4.8 \pm 0.5$ & $4.2 \pm 0.1$ \\
\hline & $\mathrm{J} 3$ & $2.5 \pm 0.4$ & $1.3 \pm 0.0$ & $1.8 \pm 0.1$ & $2.2 \pm 0.3$ & $1.4 \pm 0.0$ & $1.8 \pm 0.1$ \\
\hline & $\mathrm{J} 4$ & $1.7 \pm 0.1$ & $1.4 \pm 0.1$ & $1.7 \pm 0.1$ & $2.4 \pm 0.3$ & $2.1 \pm 0.5$ & $2.6 \pm 0.3$ \\
\hline & $\mathrm{BP}$ & $2.2 \pm 0.2$ & $1.5 \pm 0.0$ & $1.6 \pm 0.2$ & $2.4 \pm 0.4$ & $2.0 \pm 0.1$ & $2.1 \pm 0.2$ \\
\hline \multirow{4}{*}{ Trp } & $\mathrm{ZP}$ & $0.2 \pm 0.0$ & $0.2 \pm 0.0$ & $0.3 \pm 0.1$ & $0.7 \pm 0.1$ & $4.2 \pm 1.6$ & $4.5 \pm 0.7$ \\
\hline & $\mathrm{J} 3$ & $5.4 \pm 0.5$ & $6.0 \pm 1.2$ & $6.8 \pm 2.7$ & $5.9 \pm 0.2$ & $1.3 \pm 0.4$ & $1.4 \pm 0.7$ \\
\hline & $\mathrm{J} 4$ & $3.1 \pm 0.6$ & $2.4 \pm 0.7$ & $2.7 \pm 1.5$ & $7.1 \pm 2.4$ & $1.6 \pm 0.0$ & $3.8 \pm 0.2$ \\
\hline & $\mathrm{BP}$ & $8.0 \pm 3.5$ & $5.6 \pm 1.1$ & $2.5 \pm 1.0$ & $10.7 \pm 0.5$ & $4.5 \pm 0.8$ & $2.5 \pm 0.8$ \\
\hline \multirow{4}{*}{ His } & $\mathrm{ZP}$ & $0.4 \pm 0.0$ & $0.7 \pm 0.0$ & $1.6 \pm 0.0$ & $3.4 \pm 0.3$ & $5.8 \pm 0.3$ & $5.8 \pm 0.1$ \\
\hline & $\mathrm{J} 3$ & $2.1 \pm 1.2$ & $2.0 \pm 0.2$ & $3.3 \pm 0.8$ & $5.7 \pm 0.1$ & $3.2 \pm 0.5$ & $3.5 \pm 0.9$ \\
\hline & $\mathrm{J} 4$ & $1.3 \pm 0.3$ & $1.4 \pm 0.1$ & $2.2 \pm 0.6$ & $5.3 \pm 0.6$ & $4.4 \pm 1.3$ & $6.0 \pm 1.2$ \\
\hline & $\mathrm{BP}$ & $2.1 \pm 0.5$ & $2.3 \pm 0.2$ & $2.5 \pm 0.2$ & $6.1 \pm 1.6$ & $5.7 \pm 0.5$ & $5.0 \pm 0.7$ \\
\hline Cys & $\mathrm{ZP}$ & $0.6 \pm 0.1$ & $0.8 \pm 0.2$ & $0.8 \pm 0.2$ & $0.7 \pm 0.2$ & $0.7 \pm 0.1$ & $0.7 \pm 0.2$ \\
\hline
\end{tabular}




\begin{tabular}{|c|c|c|c|c|c|c|c|}
\hline compounds & variety & week 1 & week 2 & week 3 & week 4 & week 5 & week 6 \\
\hline & $\mathrm{J} 3$ & $0.5 \pm 0.1$ & $0.3 \pm 0.0$ & $0.3 \pm 0.0$ & $0.3 \pm 0.0$ & $0.2 \pm 0.0$ & $0.1 \pm 0.0$ \\
\hline & $\mathrm{J} 4$ & $0.1 \pm 0.1$ & $0.3 \pm 0.1$ & $0.3 \pm 0.1$ & $0.3 \pm 0.2$ & $0.2 \pm 0.1$ & $0.1 \pm 0.0$ \\
\hline & $\mathrm{BP}$ & $0.2 \pm 0.1$ & $0.2 \pm 0.0$ & $0.2 \pm 0.1$ & $0.2 \pm 0.0$ & $0.2 \pm 0.0$ & $0.1 \pm 0.0$ \\
\hline \multirow{4}{*}{ Ala } & $\mathrm{ZP}$ & $12.8 \pm 2.3$ & $43.8 \pm 18.2$ & $30.3 \pm 5.9$ & $79.0 \pm 28.0$ & $206.0 \pm 68.9$ & $136.6 \pm 23.2$ \\
\hline & $\mathrm{J} 3$ & $76.6 \pm 8.1$ & $38.2 \pm 2.9$ & $44.6 \pm 4.2$ & $49.6 \pm 3.7$ & $32.7 \pm 1.9$ & $41.3 \pm 3.2$ \\
\hline & $\mathrm{J} 4$ & $50.8 \pm 4.3$ & $35.1 \pm 1.0$ & $46.2 \pm 7.6$ & $64.9 \pm 10.4$ & $46.3 \pm 15.6$ & $52.5 \pm 8.5$ \\
\hline & $\mathrm{BP}$ & $85.0 \pm 11.7$ & $35.5 \pm 1.7$ & $39.8 \pm 4.7$ & $58.4 \pm 15.8$ & $40.3 \pm 4.0$ & $42.8 \pm 5.4$ \\
\hline \multirow{4}{*}{ Pro } & $\mathrm{ZP}$ & $4.6 \pm 0.6$ & $7.1 \pm 0.8$ & $15.1 \pm 1.0$ & $42.5 \pm 5.2$ & $50.2 \pm 2.4$ & $55.5 \pm 3.5$ \\
\hline & $\mathrm{J} 3$ & $9.2 \pm 0.5$ & $3.5 \pm 0.1$ & $4.9 \pm 1.5$ & $24.3 \pm 0.4$ & $14.6 \pm 1.6$ & $9.9 \pm 2.3$ \\
\hline & $\mathrm{J} 4$ & $7.2 \pm 1.1$ & $4.0 \pm 0.2$ & $4.3 \pm 0.3$ & $19.5 \pm 2.6$ & $19.5 \pm 5.1$ & $28.6 \pm 3.5$ \\
\hline & $\mathrm{BP}$ & $8.7 \pm 0.6$ & $3.9 \pm 0.1$ & $3.7 \pm 0.3$ & $23.7 \pm 6.4$ & $26.8 \pm 4.3$ & $14.1 \pm 1.7$ \\
\hline \multirow{4}{*}{ Tyr } & $\mathrm{ZP}$ & $0.4 \pm 0.2$ & $0.5 \pm 0.2$ & $1.8 \pm 0.9$ & $10.4 \pm 3.9$ & $31.5 \pm 7.3$ & $32.7 \pm 3.8$ \\
\hline & $\mathrm{J} 3$ & $7.0 \pm 0.2$ & $2.3 \pm 0.8$ & $2.9 \pm 1.4$ & $5.0 \pm 0.5$ & $1.2 \pm 0.3$ & $1.5 \pm 1.0$ \\
\hline & $\mathrm{J} 4$ & $3.9 \pm 1.4$ & $1.4 \pm 0.3$ & $1.8 \pm 1.1$ & $10.4 \pm 2.3$ & $3.0 \pm 0.1$ & $8.0 \pm 0.8$ \\
\hline & $\mathrm{BP}$ & $8.0 \pm 2.5$ & $1.6 \pm 0.1$ & $0.7 \pm 0.5$ & $14 \pm 1.4$ & $5.6 \pm 2.0$ & $2.8 \pm 1.2$ \\
\hline \multirow{4}{*}{ Asp } & $\mathrm{ZP}$ & $54.4 \pm 2.0$ & $27.0 \pm 19.9$ & $50.4 \pm 1.2$ & $48.4 \pm 4.1$ & $48.4 \pm 1.6$ & $68.9 \pm 0.4$ \\
\hline & $\mathrm{J} 3$ & $42.0 \pm 7.5$ & $34.0 \pm 1.1$ & $42.0 \pm 2.8$ & $43.5 \pm 2.5$ & $38 \pm 3.6$ & $44.8 \pm 2.5$ \\
\hline & $\mathrm{J} 4$ & $39.7 \pm 2.2$ & $29.6 \pm 1.3$ & $35.7 \pm 1.8$ & $45.7 \pm 0.5$ & $42.9 \pm 5.5$ & $51.0 \pm 0.2$ \\
\hline & $\mathrm{BP}$ & $37.7 \pm 9.6$ & $35.0 \pm 1.1$ & $34.2 \pm 0.5$ & $43.0 \pm 1.6$ & $42.6 \pm 3.8$ & $53.6 \pm 3.1$ \\
\hline \multirow{4}{*}{ Asn } & $\mathrm{ZP}$ & $101.0 \pm 3.9$ & $99.0 \pm 4.9$ & $202.8 \pm 16.9$ & $238.5 \pm 29.8$ & $412.7 \pm 44.4$ & $426.0 \pm 31.8$ \\
\hline & $\mathrm{J} 3$ & $305.2 \pm 16$ & $195.7 \pm 8.2$ & $193.6 \pm 60.7$ & $222.2 \pm 6.5$ & $35.4 \pm 11.9$ & $137.3 \pm 27.6$ \\
\hline & $\mathrm{J} 4$ & $166.1 \pm 10.6$ & $204.3 \pm 3.7$ & $182.7 \pm 5$ & $247.9 \pm 40.5$ & $72.9 \pm 11.1$ & $154.2 \pm 24.7$ \\
\hline & $\mathrm{BP}$ & $267.5 \pm 8.6$ & $136.7 \pm 16.0$ & $98.1 \pm 19.2$ & $148.0 \pm 39.4$ & $82.1 \pm 21.3$ & $181.7 \pm 60.4$ \\
\hline \multirow{4}{*}{$\operatorname{Arg}$} & $\mathrm{ZP}$ & $1503.2 \pm 48.9$ & $2045.5 \pm 47.6$ & $3058.0 \pm 92.4$ & $3848.0 \pm 138.8$ & $4597.5 \pm 159.1$ & $4957.4 \pm 77.3$ \\
\hline & $\mathrm{J} 3$ & $1202 \pm 128.8$ & $883.8 \pm 75.7$ & $1377.6 \pm 267.2$ & $2274.3 \pm 60.9$ & $1212.4 \pm 207.5$ & $1825.6 \pm 104.4$ \\
\hline & $\mathrm{J} 4$ & $685.8 \pm 138.1$ & $868.2 \pm 65.0$ & $1174.4 \pm 118.9$ & $2092.5 \pm 252.1$ & $1606.5 \pm 431.2$ & $2146.6 \pm 140.2$ \\
\hline & $\mathrm{BP}$ & $1158.2 \pm 168.3$ & $876.7 \pm 22.5$ & $1026.6 \pm 49.4$ & $1832.4 \pm 193.8$ & $1571.9 \pm 175.3$ & $2280.4 \pm 204.4$ \\
\hline \multirow{4}{*}{ Gln } & $\mathrm{ZP}$ & $85.5 \pm 20.7$ & $39.6 \pm 14.6$ & $66.5 \pm 24.9$ & $79.2 \pm 40.3$ & $155.4 \pm 89.9$ & $83.8 \pm 2.9$ \\
\hline & $\mathrm{J} 3$ & $385.1 \pm 91.4$ & $232.6 \pm 20.2$ & $137.4 \pm 10.5$ & $83.4 \pm 8.2$ & $12.8 \pm 3.6$ & $17.2 \pm 3.8$ \\
\hline & $\mathrm{J} 4$ & $175.4 \pm 7.5$ & $170.3 \pm 10.5$ & $120.0 \pm 12$ & $111.3 \pm 14.6$ & $60.9 \pm 18.9$ & $61.8 \pm 4.9$ \\
\hline & $\mathrm{BP}$ & $320.6 \pm 52.7$ & $152.2 \pm 26.8$ & $77.5 \pm 7.7$ & $80.7 \pm 25.4$ & $48.8 \pm 9.9$ & $37.9 \pm 4.2$ \\
\hline \multirow{4}{*}{ Glu } & $\mathrm{ZP}$ & $137.0 \pm 5.4$ & $131.7 \pm 24.4$ & $105.7 \pm 5.1$ & $147.2 \pm 23.9$ & $222.2 \pm 25.4$ & $204.3 \pm 8.8$ \\
\hline & $\mathrm{J} 3$ & $257.9 \pm 11.7$ & $164.8 \pm 4.7$ & $142.5 \pm 7.2$ & $124.1 \pm 3.9$ & $84.6 \pm 2.9$ & $94.0 \pm 7.3$ \\
\hline & $\mathrm{J} 4$ & $153.9 \pm 13.0$ & $141.7 \pm 13.0$ & $123.8 \pm 7.4$ & $113.7 \pm 3.9$ & $99.5 \pm 23.9$ & $113.1 \pm 9.2$ \\
\hline & $\mathrm{BP}$ & $193.0 \pm 6.1$ & $126.2 \pm 1.5$ & $105.3 \pm 2.3$ & $109.4 \pm 11.6$ & $82.9 \pm 3.9$ & $86.6 \pm 3.4$ \\
\hline \multirow{4}{*}{ Met } & $\mathrm{ZP}$ & $1.3 \pm 0.2$ & $1.3 \pm 0.1$ & $1.6 \pm 0.2$ & $6.0 \pm 1.2$ & $11.7 \pm 0.7$ & $12.7 \pm 1.5$ \\
\hline & $\mathrm{J} 3$ & $6.5 \pm 0.1$ & $1.7 \pm 0.0$ & $0.9 \pm 0.1$ & $1.2 \pm 0.0$ & $1.3 \pm 0.2$ & $1.3 \pm 0.0$ \\
\hline & $\mathrm{J} 4$ & $4.2 \pm 0.5$ & $1.5 \pm 0.0$ & $0.8 \pm 0.1$ & $1.4 \pm 0.1$ & $1.6 \pm 0.3$ & $2.0 \pm 0.4$ \\
\hline & $\mathrm{BP}$ & $5.5 \pm 0.4$ & $1.5 \pm 0.0$ & $0.8 \pm 0.0$ & $1.2 \pm 0.2$ & $1.2 \pm 0.1$ & $1.3 \pm 0.2$ \\
\hline \multirow{4}{*}{ Val } & $\mathrm{ZP}$ & $23.3 \pm 3.0$ & $23.7 \pm 2.2$ & $28 \pm 2.9$ & $59.2 \pm 8.0$ & $100.2 \pm 14.7$ & $94.4 \pm 0.8$ \\
\hline & $\mathrm{J} 3$ & $57.2 \pm 4.7$ & $27.6 \pm 1.4$ & $29 \pm 2.5$ & $32.2 \pm 1.7$ & $18.3 \pm 2.1$ & $21.1 \pm 3.6$ \\
\hline & $\mathrm{J} 4$ & $35.0 \pm 1.3$ & $23.6 \pm 0.9$ & $23.3 \pm 2.4$ & $34.2 \pm 3.5$ & $24.2 \pm 6.9$ & $35.3 \pm 4.8$ \\
\hline & $\mathrm{BP}$ & $52.0 \pm 4.9$ & $25.7 \pm 0.3$ & $21.1 \pm 0.7$ & $35.7 \pm 7.7$ & $27 \pm 1.7$ & $29.0 \pm 3.5$ \\
\hline \multirow{3}{*}{ Ser } & $\mathrm{ZP}$ & $22.1 \pm 2.3$ & $31.2 \pm 2.9$ & $45.6 \pm 4.9$ & $90.4 \pm 16.2$ & $130.4 \pm 21.9$ & $133.3 \pm 8.2$ \\
\hline & $\mathrm{J} 3$ & $58.1 \pm 9.1$ & $26.8 \pm 1.4$ & $43.8 \pm 2.8$ & $53.6 \pm 3.5$ & $46.6 \pm 5.9$ & $59.6 \pm 8.1$ \\
\hline & $\mathrm{J} 4$ & $40.3 \pm 5.0$ & $25.1 \pm 1.0$ & $40.2 \pm 2.1$ & $63.4 \pm 7.0$ & $69.6 \pm 17.1$ & $77.8 \pm 10.2$ \\
\hline
\end{tabular}




\begin{tabular}{|c|c|c|c|c|c|c|c|}
\hline compounds & variety & week 1 & week 2 & week 3 & week 4 & week 5 & week 6 \\
\hline & $\mathrm{BP}$ & $62.2 \pm 11$ & $27.2 \pm 0.8$ & $37.4 \pm 4.5$ & $72.3 \pm 16.2$ & $59.7 \pm 4.7$ & $70.3 \pm 1.8$ \\
\hline \multirow{4}{*}{ Leu } & $\mathrm{ZP}$ & $5.0 \pm 0.7$ & $4.6 \pm 1.2$ & $4.8 \pm 1.3$ & $16.1 \pm 3.8$ & $32.0 \pm 7.7$ & $25.3 \pm 2.4$ \\
\hline & $\mathrm{J} 3$ & $25.6 \pm 8.0$ & $10.2 \pm 0.5$ & $6.8 \pm 1.3$ & $6.8 \pm 0.5$ & $3.4 \pm 0.2$ & $3.0 \pm 0.5$ \\
\hline & $\mathrm{J} 4$ & $10.9 \pm 0.8$ & $7.3 \pm 0.5$ & $5.2 \pm 0.7$ & $8.3 \pm 0.5$ & $4.9 \pm 2.2$ & $6.9 \pm 1.9$ \\
\hline & $\mathrm{BP}$ & $18.2 \pm 4.4$ & $8.3 \pm 0.3$ & $4.3 \pm 0.2$ & $8.7 \pm 3.1$ & $7.0 \pm 1.5$ & $3.6 \pm 0.5$ \\
\hline \multirow{4}{*}{ Ile } & $\mathrm{ZP}$ & $6.4 \pm 0.8$ & $6.0 \pm 0.8$ & $5.3 \pm 0.9$ & $16.0 \pm 2.9$ & $28.4 \pm 4.7$ & $23.3 \pm 1.6$ \\
\hline & $\mathrm{J} 3$ & $18.0 \pm 3.3$ & $9.0 \pm 0.3$ & $7.5 \pm 1.5$ & $8.2 \pm 0.4$ & $4.1 \pm 0.4$ & $3.9 \pm 0.9$ \\
\hline & $\mathrm{J} 4$ & $10.9 \pm 0.6$ & $6.7 \pm 0.0$ & $5.6 \pm 0.8$ & $8.7 \pm 0.9$ & $5.8 \pm 2.4$ & $9.4 \pm 1.7$ \\
\hline & $\mathrm{BP}$ & $15.0 \pm 2.1$ & $7.4 \pm 0.3$ & $4.9 \pm 0.3$ & $9.3 \pm 3.0$ & $7.5 \pm 1.4$ & $4.8 \pm 1.7$ \\
\hline \multirow{4}{*}{ Phe } & $\mathrm{ZP}$ & $4.9 \pm 0.4$ & $5.9 \pm 1.5$ & $3.3 \pm 0.8$ & $11.8 \pm 3.1$ & $17.0 \pm 5.7$ & $12.4 \pm 1.8$ \\
\hline & $\mathrm{J} 3$ & $56.9 \pm 10.8$ & $35.3 \pm 4.6$ & $41.4 \pm 7.5$ & $18.2 \pm 0.7$ & $12.3 \pm 0.3$ & $4.8 \pm 1.2$ \\
\hline & $\mathrm{J} 4$ & $35.6 \pm 7.8$ & $22.0 \pm 0.8$ & $27.1 \pm 7.2$ & $19.8 \pm 2.4$ & $12.4 \pm 4.0$ & $9.7 \pm 4.6$ \\
\hline & $\mathrm{BP}$ & $52.8 \pm 5.9$ & $30.1 \pm 2.9$ & $27.0 \pm 4.0$ & $19.3 \pm 5.6$ & $16.7 \pm 4.4$ & $4.7 \pm 1.2$ \\
\hline \multirow{4}{*}{ Thr } & $\mathrm{ZP}$ & $10.1 \pm 0.8$ & $10.8 \pm 1.2$ & $16.2 \pm 1.4$ & $31.7 \pm 4.1$ & $45.0 \pm 3.8$ & $45.6 \pm 1.3$ \\
\hline & $\mathrm{J} 3$ & $21.4 \pm 5.5$ & $10.9 \pm 0.5$ & $14.8 \pm 2.3$ & $30.8 \pm 0.2$ & $20.5 \pm 2.1$ & $27.8 \pm 4.7$ \\
\hline & $\mathrm{J} 4$ & $12.6 \pm 0.2$ & $10.0 \pm 0.4$ & $13.4 \pm 1.3$ & $30.6 \pm 4.4$ & $31.6 \pm 9.4$ & $40.5 \pm 5.7$ \\
\hline & $\mathrm{BP}$ & $19.9 \pm 3.8$ & $10.2 \pm 0.7$ & $12.0 \pm 1.3$ & $40.7 \pm 12.6$ & $33.3 \pm 1.3$ & $39.5 \pm 2.6$ \\
\hline \multirow{4}{*}{ Lys } & $\mathrm{ZP}$ & $56.3 \pm 5.4$ & $73.5 \pm 2.3$ & $117.8 \pm 6.0$ & $157.3 \pm 8.2$ & $239.9 \pm 15.6$ & $251.0 \pm 5.4$ \\
\hline & $\mathrm{J} 3$ & $82.8 \pm 17.4$ & $71.2 \pm 1.3$ & $103.8 \pm 20.9$ & $200.7 \pm 13.4$ & $133.2 \pm 4.7$ & $171.0 \pm 17.3$ \\
\hline & $\mathrm{J} 4$ & $65.6 \pm 7.2$ & $63.9 \pm 5.2$ & $85.5 \pm 15.4$ & $177.6 \pm 12.5$ & $162.6 \pm 39.4$ & $212.8 \pm 26.1$ \\
\hline & $\mathrm{BP}$ & $87.8 \pm 10.3$ & $79.7 \pm 6.9$ & $92.9 \pm 1.2$ & $184.3 \pm 26.5$ & $158.4 \pm 14.5$ & $199.7 \pm 16.9$ \\
\hline \multirow{4}{*}{ GABA } & $\mathrm{ZP}$ & $1.0 \pm 0.1$ & $1.1 \pm 0.0$ & $1.0 \pm 0.1$ & $1.3 \pm 0.3$ & $8.5 \pm 3.9$ & $3.5 \pm 0.7$ \\
\hline & $\mathrm{J} 3$ & $4.7 \pm 0.5$ & $1.9 \pm 0.1$ & $2.2 \pm 0.2$ & $2.0 \pm 0.1$ & $1.3 \pm 0.2$ & $1.3 \pm 0.1$ \\
\hline & $\mathrm{J} 4$ & $3.5 \pm 0.8$ & $2.3 \pm 0.2$ & $2.9 \pm 0.5$ & $2.3 \pm 0.3$ & $1.8 \pm 0.5$ & $1.8 \pm 0.4$ \\
\hline & $\mathrm{BP}$ & $4.1 \pm 0.2$ & $2.2 \pm 0.2$ & $2.5 \pm 0.3$ & $2.5 \pm 0.4$ & $1.5 \pm 0.3$ & $1.4 \pm 0.1$ \\
\hline
\end{tabular}

Table S4 Agro-morphological traits of garlic in different growing stages $(n=3)$

\begin{tabular}{|c|c|c|c|c|c|c|c|c|}
\hline \multirow{3}{*}{ Variety } & Stage & $\begin{array}{c}\text { Bulb height } \\
(\mathrm{mm})\end{array}$ & $\begin{array}{c}\text { Bulb } \\
\text { diameter } \\
(\mathrm{mm})\end{array}$ & $\begin{array}{c}\text { Bulb weight } \\
(\mathrm{mm})\end{array}$ & $\begin{array}{c}\text { Clove } \\
\text { height } \\
(\mathrm{mm})\end{array}$ & $\begin{array}{c}\text { Clove } \\
\text { width } \\
(\mathrm{mm})\end{array}$ & $\begin{array}{c}\text { Clove } \\
\text { weight } \\
(\mathrm{g})\end{array}$ & $\begin{array}{c}\text { Moisture } \\
\text { content }(\%)\end{array}$ \\
\hline \multirow{6}{*}{ ZP } & Week 0 & $31.1 \pm 0.8$ & $31.8 \pm 0.8$ & $14.6 \pm 0.9$ & $16.2 \pm 0.1$ & $8.0 \pm 0.1$ & $0.6 \pm 0.1$ & $69.7 \pm 0.4$ \\
\cline { 2 - 9 } & week 1 & $33.9 \pm 0.8$ & $38.8 \pm 1.2$ & $23.8 \pm 2.0$ & $20.7 \pm 0.7$ & $10.1 \pm 0.6$ & $1.3 \pm 0.0$ & $68.8 \pm 1.1$ \\
\cline { 2 - 9 } & week 2 & $36.6 \pm 0.3$ & $43.7 \pm 0.3$ & $31.3 \pm 0.8$ & $24.6 \pm 0.5$ & $12.9 \pm 0.8$ & $2.4 \pm 0.1$ & $67.0 \pm 0.1$ \\
\cline { 2 - 9 } & week 3 & $36.8 \pm 0.7$ & $45.3 \pm 0.7$ & $32.4 \pm 1.5$ & $26.2 \pm 0.1$ & $14.1 \pm 0.2$ & $2.8 \pm 0.1$ & $64.7 \pm 1.1$ \\
\cline { 2 - 9 } & week 4 & $36.2 \pm 0.1$ & $42.3 \pm 1.0$ & $27.2 \pm 1.6$ & $25.9 \pm 0.2$ & $14.3 \pm 0.5$ & $2.6 \pm 0.1$ & $62.6 \pm 0.6$ \\
\cline { 2 - 9 } & week 5 & $39.8 \pm 0.4$ & $46.7 \pm 0.5$ & $34.2 \pm 1.8$ & $27.8 \pm 0.6$ & $15.1 \pm 1.0$ & $3.9 \pm 0.2$ & $67.2 \pm 1.0$ \\
\cline { 2 - 9 } & week 6 & $39.1 \pm 0.6$ & $47.9 \pm 1.8$ & $34.4 \pm 2.2$ & $27.8 \pm 0.3$ & $15.1 \pm 0.6$ & $3.9 \pm 0.2$ & $68.4 \pm 0.8$ \\
\hline \multirow{5}{*}{ J3 } & week 1 & $36.3 \pm 0.9$ & $44.8 \pm 3.3$ & $35.3 \pm 3.4$ & $13.9 \pm 1.2$ & $7.3 \pm 0.6$ & $0.6 \pm 0.0$ & $77.0 \pm 2.4$ \\
\cline { 2 - 9 } & week 2 & $39.5 \pm 2.0$ & $47.8 \pm 0.9$ & $43.1 \pm 3.4$ & $21.9 \pm 0.9$ & $9.9 \pm 0.2$ & $1.4 \pm 0.1$ & $70.3 \pm 0.8$ \\
\cline { 2 - 9 } & week 3 & $42.4 \pm 0.9$ & $54.0 \pm 1.1$ & $56.1 \pm 3.9$ & $26.1 \pm 0.6$ & $12.0 \pm 0.5$ & $2.6 \pm 0.1$ & $68.0 \pm 0.5$ \\
\cline { 2 - 9 } & week 4 & $41.9 \pm 0.5$ & $55.9 \pm 0.6$ & $59.4 \pm 0.8$ & $27.7 \pm 0.3$ & $14.0 \pm 0.3$ & $3.5 \pm 0.2$ & $64.8 \pm 1.0$ \\
\cline { 2 - 9 } & week 5 & $42.9 \pm 0.3$ & $56.8 \pm 0.8$ & $63.2 \pm 2.1$ & $28.6 \pm 0.0$ & $14.4 \pm 0.4$ & $4.3 \pm 0.2$ & $62.9 \pm 0.5$ \\
\cline { 2 - 9 } & week 6 & $44.9 \pm 0.2$ & $60.4 \pm 0.9$ & $69.9 \pm 3.0$ & $30.0 \pm 0.6$ & $15.4 \pm 0.3$ & $4.4 \pm 0.1$ & $64.6 \pm 0.7$ \\
\hline \multirow{2}{*}{ J4 } & week 1 & $40.6 \pm 1.4$ & $49.4 \pm 2.1$ & $46.8 \pm 5.5$ & $21.9 \pm 1.9$ & $9.8 \pm 0.9$ & $1.3 \pm 0.1$ & $69.8 \pm 0.3$ \\
\hline
\end{tabular}




\begin{tabular}{|c|c|c|c|c|c|c|c|c|}
\hline \multirow{7}{*}{} & week 2 & $43.2 \pm 0.6$ & $52.0 \pm 0.6$ & $53.5 \pm 1.9$ & $24.9 \pm 0.4$ & $12.6 \pm 0.7$ & $2.1 \pm 0.1$ & $69.7 \pm 0.4$ \\
\cline { 2 - 9 } & week 3 & $46.1 \pm 0.1$ & $57.3 \pm 1.3$ & $66.0 \pm 4.2$ & $28.7 \pm 0.3$ & $13.3 \pm 0.1$ & $3.3 \pm 0.4$ & $66.7 \pm 0.8$ \\
\cline { 2 - 9 } & week 4 & $43.6 \pm 1.3$ & $59.8 \pm 0.6$ & $65.3 \pm 5.5$ & $29.7 \pm 0.6$ & $15.5 \pm 0.2$ & $4.2 \pm 0.3$ & $65.9 \pm 0.9$ \\
\cline { 2 - 9 } & week 5 & $44.5 \pm 1.0$ & $58.1 \pm 0.9$ & $67.9 \pm 3.3$ & $30.9 \pm 1.0$ & $15.5 \pm 0.6$ & $4.6 \pm 0.5$ & $63.9 \pm 1.0$ \\
\cline { 2 - 9 } & week 6 & $44.1 \pm 2.0$ & $58.5 \pm 3.2$ & $66.8 \pm 9.7$ & $30.5 \pm 1.3$ & $15 \pm 0.1$ & $4.6 \pm 0.2$ & $64.0 \pm 0.5$ \\
\hline \multirow{6}{*}{ BP } & week 1 & $36.7 \pm 0.5$ & $46.5 \pm 0.6$ & $39.0 \pm 1.2$ & $17.3 \pm 0.2$ & $8.2 \pm 0.6$ & $0.8 \pm 0.0$ & $75.3 \pm 0.1$ \\
\cline { 2 - 9 } & week 2 & $41.7 \pm 0.9$ & $49.9 \pm 1.6$ & $49.2 \pm 3.9$ & $22.8 \pm 0.7$ & $10.2 \pm 0.5$ & $1.9 \pm 0.2$ & $70.1 \pm 0.1$ \\
\cline { 2 - 9 } & week 3 & $45.0 \pm 0.5$ & $55.9 \pm 0.7$ & $62.9 \pm 2.8$ & $27.1 \pm 0.7$ & $12.4 \pm 0.4$ & $2.8 \pm 0.2$ & $67.9 \pm 0.8$ \\
\cline { 2 - 9 } & week 4 & $43.2 \pm 1.2$ & $57.7 \pm 0.3$ & $64.3 \pm 2.0$ & $28.7 \pm 0.3$ & $14.0 \pm 1.0$ & $4.0 \pm 0.5$ & $66.0 \pm 1.0$ \\
\cline { 2 - 9 } & week 5 & $44.4 \pm 0.5$ & $55.4 \pm 1.3$ & $60.5 \pm 2.8$ & $29.8 \pm 1.2$ & $14.9 \pm 0.6$ & $4.2 \pm 0.0$ & $64.9 \pm 1.2$ \\
\cline { 2 - 8 } & week 6 & $42.4 \pm 0.6$ & $55.5 \pm 1.1$ & $55.4 \pm 2.9$ & $29.5 \pm 0.2$ & $14.5 \pm 0.2$ & $4.0 \pm 0.1$ & $65.8 \pm 0.5$ \\
\hline
\end{tabular}

Table S5 Parameters of OPLS regression models for characteristic components in garlic based on agro-morphological traits

\begin{tabular}{|c|c|c|c|c|c|}
\hline Variety & Compounds & $\mathrm{R}^{2}$ & $\mathrm{Q}^{2}$ & RMSEE & RMSEcv \\
\hline \multirow{3}{*}{ ZP } & Allicin & 0.950 & 0.892 & 64.385 & 80.267 \\
\cline { 2 - 6 } & Total 7 flavor precursors & 0.926 & 0.850 & 426.802 & 513.17 \\
\hline \multirow{2}{*}{ J3 } & Allicin & 0.888 & 0.844 & 91.745 & 93.671 \\
\cline { 2 - 6 } & Total 7 flavor precursors & 0.717 & 0.546 & 478.947 & 525.416 \\
\hline \multirow{3}{*}{ J4 } & Allicin & 0.854 & 0.691 & 110.682 & 131.551 \\
\cline { 2 - 6 } & Total 7 flavor precursors & 0.889 & 0.519 & 300.447 & 477.128 \\
\hline \multirow{2}{*}{ BP } & Allicin & 0.872 & 0.833 & 92.009 & 90.877 \\
\cline { 2 - 6 } & Total 7 flavor precursors & 0.863 & 0.746 & 306.311 & 340.279 \\
\hline
\end{tabular}

Table S6 The observed and predicted concentrations and relative error (RE) between them for testing set predicted by OPLS regression models

\begin{tabular}{|c|c|c|c|c|c|}
\hline Variety & Compounds & Sampling time & $\begin{array}{l}\text { Observations } \\
\mathrm{mg} / 100 \mathrm{~g} \mathrm{dw}\end{array}$ & $\begin{array}{l}\text { Predictions } \\
\mathrm{mg} / 100 \mathrm{~g} \mathrm{dw}\end{array}$ & $\begin{array}{l}\mathrm{RE} \\
(\%)\end{array}$ \\
\hline \multirow{7}{*}{$\mathrm{ZP}$} & \multirow{7}{*}{ allicin } & week 1 & 313.55 & 352.05 & 12.28 \\
\hline & & week 2 & 465.98 & 306.51 & 34.22 \\
\hline & & week 3 & 497.72 & 537.89 & 8.07 \\
\hline & & week 4 & 586.77 & 611.98 & 4.30 \\
\hline & & week 5 & 782.99 & 668.71 & 14.60 \\
\hline & & week 6 & 1022.90 & 847.93 & 17.11 \\
\hline & & week 7 & 1026.06 & 871.78 & 15.04 \\
\hline \multirow{7}{*}{$\mathrm{ZP}$} & \multirow{7}{*}{ Total 7 flavor precursors } & week 1 & 912.63 & 905.09 & 0.83 \\
\hline & & week 2 & 1533.76 & 934.19 & 39.09 \\
\hline & & week 3 & 1747.55 & 2196.38 & 25.68 \\
\hline & & week 4 & 2591.55 & 2645.47 & 2.08 \\
\hline & & week 5 & 3231.44 & 2849.95 & 11.81 \\
\hline & & week 6 & 4079.05 & 3822.74 & 6.28 \\
\hline & & week 7 & 4253.95 & 3942.43 & 7.32 \\
\hline \multirow{6}{*}{$\mathrm{J} 3$} & \multirow{6}{*}{ allicin } & week 1 & 4553.22 & 3707.33 & 18.58 \\
\hline & & week 2 & 2635.13 & 2830.00 & 7.40 \\
\hline & & week 3 & 3559.87 & 3273.46 & 8.05 \\
\hline & & week 4 & 3968.94 & 4061.36 & 2.33 \\
\hline & & week 5 & 4051.69 & 3602.76 & 11.08 \\
\hline & & week 6 & 4920.37 & 4015.98 & 18.38 \\
\hline
\end{tabular}




\begin{tabular}{|c|c|c|c|c|c|}
\hline Variety & Compounds & Sampling time & $\begin{array}{l}\text { Observations } \\
\mathrm{mg} / 100 \mathrm{~g} \mathrm{dw}\end{array}$ & $\begin{array}{l}\text { Predictions } \\
\mathrm{mg} / 100 \mathrm{~g} \mathrm{dw}\end{array}$ & $\begin{array}{l}\mathrm{RE} \\
(\%)\end{array}$ \\
\hline \multirow{6}{*}{$\mathrm{J} 3$} & \multirow{6}{*}{ Total 7 flavor precursors } & week 1 & 664.69 & 526.35 & 20.81 \\
\hline & & week 2 & 692.51 & 617.06 & 10.89 \\
\hline & & week 3 & 852.87 & 839.20 & 1.60 \\
\hline & & week 4 & 965.79 & 987.54 & 2.25 \\
\hline & & week 5 & 1038.26 & 945.57 & 8.93 \\
\hline & & week 6 & 1213.31 & 1114.78 & 8.12 \\
\hline \multirow{6}{*}{$\mathrm{J} 4$} & \multirow{6}{*}{ allicin } & week 1 & 641.12 & 819.08 & 27.76 \\
\hline & & week 2 & 752.81 & 625.09 & 16.97 \\
\hline & & week 3 & 727.06 & 678.17 & 6.72 \\
\hline & & week 4 & 1000.25 & 1333.69 & 33.34 \\
\hline & & week 5 & 1192.99 & 1166.54 & 2.22 \\
\hline & & week 6 & 1360.00 & 1145.98 & 15.74 \\
\hline \multirow{6}{*}{$\mathrm{J} 4$} & \multirow{6}{*}{ Total 7 flavor precursors } & week 1 & 3025.74 & 3505.11 & 15.84 \\
\hline & & week 2 & 3203.09 & 2640.15 & 17.57 \\
\hline & & week 3 & 3071.72 & 2416.09 & 21.34 \\
\hline & & week 4 & 3693.09 & 5158.36 & 39.68 \\
\hline & & week 5 & 5191.82 & 4152.39 & 20.02 \\
\hline & & week 6 & 4598.70 & 3905.64 & 15.07 \\
\hline \multirow{6}{*}{$\mathrm{BP}$} & \multirow{6}{*}{ allicin } & week 1 & 688.15 & 705.04 & 2.45 \\
\hline & & week 2 & 816.30 & 764.27 & 6.37 \\
\hline & & week 3 & 792.80 & 745.15 & 6.01 \\
\hline & & week 4 & 1018.63 & 1191.43 & 16.96 \\
\hline & & week 5 & 1084.77 & 1161.94 & 7.11 \\
\hline & & week 6 & 1470.69 & 1206.55 & 17.96 \\
\hline \multirow{6}{*}{$\mathrm{BP}$} & \multirow{6}{*}{ Total 7 flavor precursors } & week 1 & 3262.82 & 3430.85 & 5.15 \\
\hline & & week 2 & 2407.16 & 2644.65 & 9.87 \\
\hline & & week 3 & 2356.43 & 2560.76 & 8.67 \\
\hline & & week 4 & 4037.02 & 4512.33 & 11.77 \\
\hline & & week 5 & 4063.72 & 3425.81 & 15.70 \\
\hline & & week 6 & 4965.52 & 4268.08 & 14.05 \\
\hline
\end{tabular}

Table S7 The VIP values for agro-morphological traits in OPLS regression models for allicin and total 7 flavor precursors in ZP, $\mathrm{J} 3, \mathrm{~J} 4$, and BP

\begin{tabular}{|c|c|c|c|}
\hline agro-morphological traits & VIP & agro-morphological traits & VIP \\
\hline \multicolumn{2}{|l|}{ ZP (Allicin) } & \multicolumn{2}{|c|}{ ZP (Total 7 flavor precursors) } \\
\hline Clove weight & 1.20 & Clove weight & 1.17 \\
\hline Clove width & 1.13 & Clove width & 1.12 \\
\hline Bulb height & 1.13 & Bulb height & 1.11 \\
\hline Clove height & 1.05 & Clove height & 1.05 \\
\hline Bulb diameter & 0.99 & Bulb diameter & 1.01 \\
\hline Bulb weight & 0.91 & Bulb weight & 0.94 \\
\hline Moisture content & 0.33 & Moisture content & 0.37 \\
\hline \multicolumn{2}{|l|}{ J3 (Allicin) } & \multicolumn{2}{|c|}{ J3 (Total 7 flavor precursors) } \\
\hline Bulb diameter & 1.08 & Bulb diameter & 1.28 \\
\hline Bulb weight & 1.06 & Bulb weight & 1.17 \\
\hline
\end{tabular}




\begin{tabular}{|c|c|c|c|}
\hline Clove weight & 1.03 & Clove weight & 1.16 \\
\hline Clove width & 1.02 & Clove width & 1.07 \\
\hline Bulb height & 0.98 & Bulb height & 0.81 \\
\hline Clove height & 0.94 & Clove height & 0.69 \\
\hline Moisture content & 0.86 & Moisture content & 0.63 \\
\hline \multicolumn{2}{|c|}{ J4 (Allicin) } & & J4 (Total 7 flavor precursors) \\
\hline Clove weight & 1.13 & Bulb diameter & 1.17 \\
\hline Moisture content & 1.11 & Clove width & 1.16 \\
\hline Clove height & 1.06 & Clove weight & 1.14 \\
\hline Clove width & 1.03 & Clove height & 1.03 \\
\hline Bulb diameter & 1.03 & Moisture content & 1.00 \\
\hline Bulb weight & 0.97 & Bulb weight & 0.94 \\
\hline Bulb height & 0.57 & Bulb height & 0.20 \\
\hline BP (Allicin) & & BP (Total 7 flavor precursors) \\
\hline Clove width & 1.33 & Clove weight & 1.55 \\
\hline Clove weight & 1.30 & Clove width & 1.45 \\
\hline Clove height & 1.15 & Clove height & 1.06 \\
\hline Moisture content & 1.10 & Moisture content & 0.88 \\
\hline Bulb diameter & 0.77 & Bulb diameter & 0.56 \\
\hline Bulb height & 0.46 & Bulb height & 0.51 \\
\hline Bulb weight & 0.45 & Bulb weight & 0.15 \\
\hline
\end{tabular}

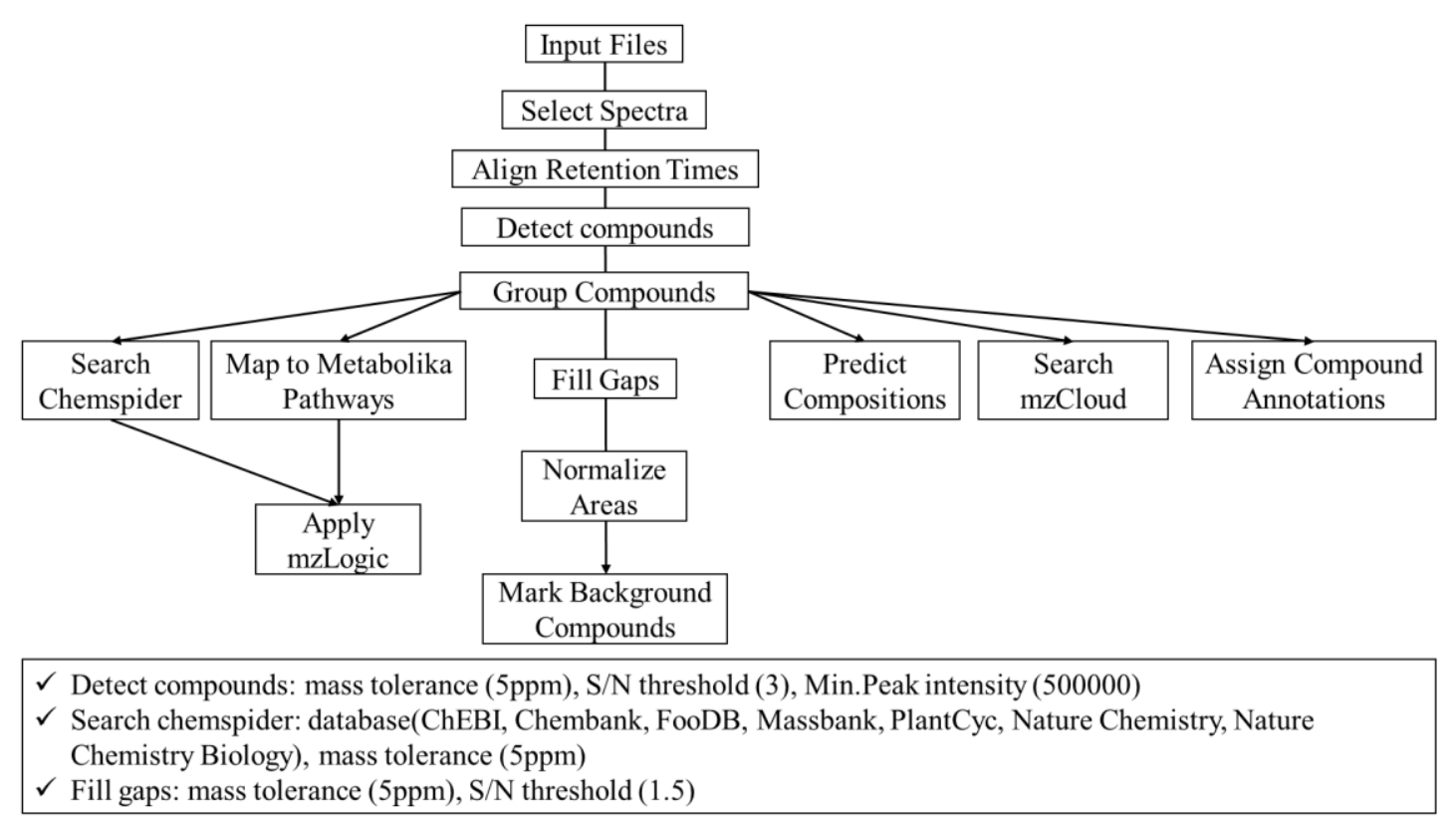

Figure S1 Workflows and parameters of Compound Discovery 3.0 analysis 
No. 33 4-Guanidinobutyric acid

RAWFILE(top): BP_0419_1 (F1)\#1358, RT=4.569 min, MS2, FTMS (+), (HCD, DDA, 146.0922@30,+1)

REFERENCE(bottom): mzCloud library, 4-Guanidinobutyric acid, C5 H11 N3 O2, MS2, FTMS, (HCD, 146.0924@40)

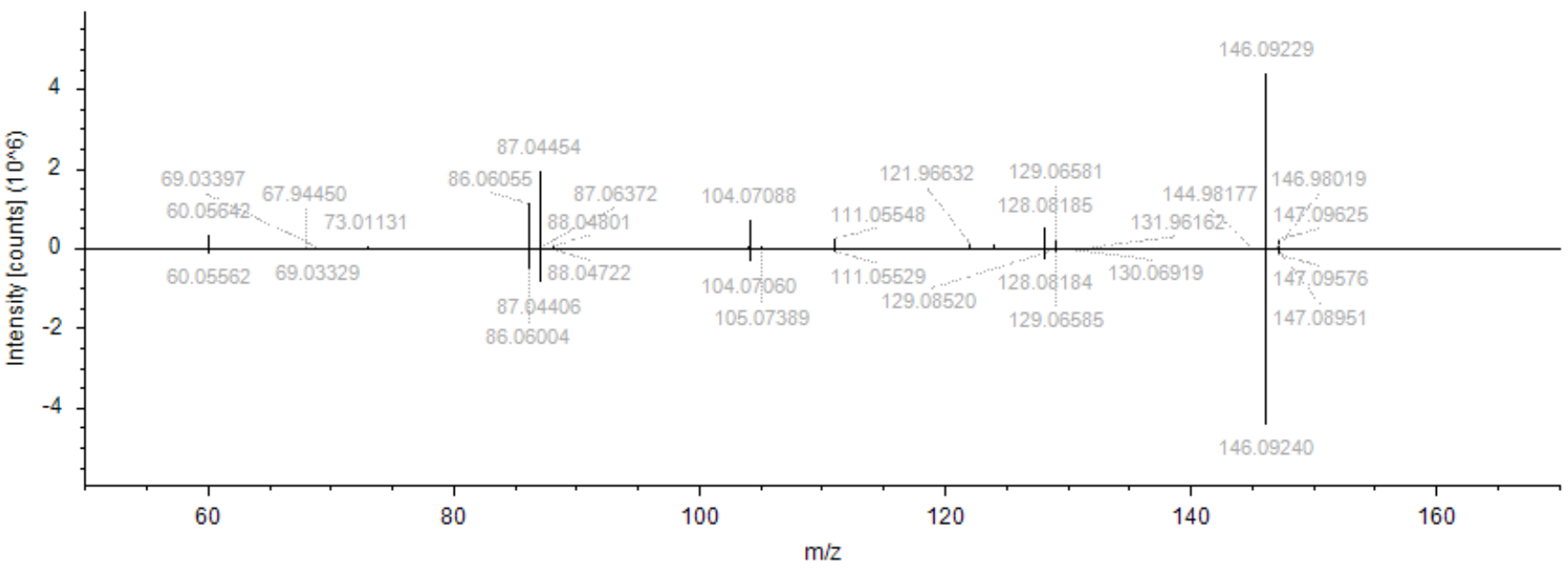

No. 37 Ergothioneine

RAWFILE(top): BP_0419_3 (F3) \#2486, RT=8.389 min, MS2, FTMS (+), (HCD, DDA, 230.0955@30, +1)

REFERENCE(bottom): mzCloud library, L-Ergothioneine, C9 H15 N3 O2 S, MS2, FTMS, (HCD, 230.0958@40)

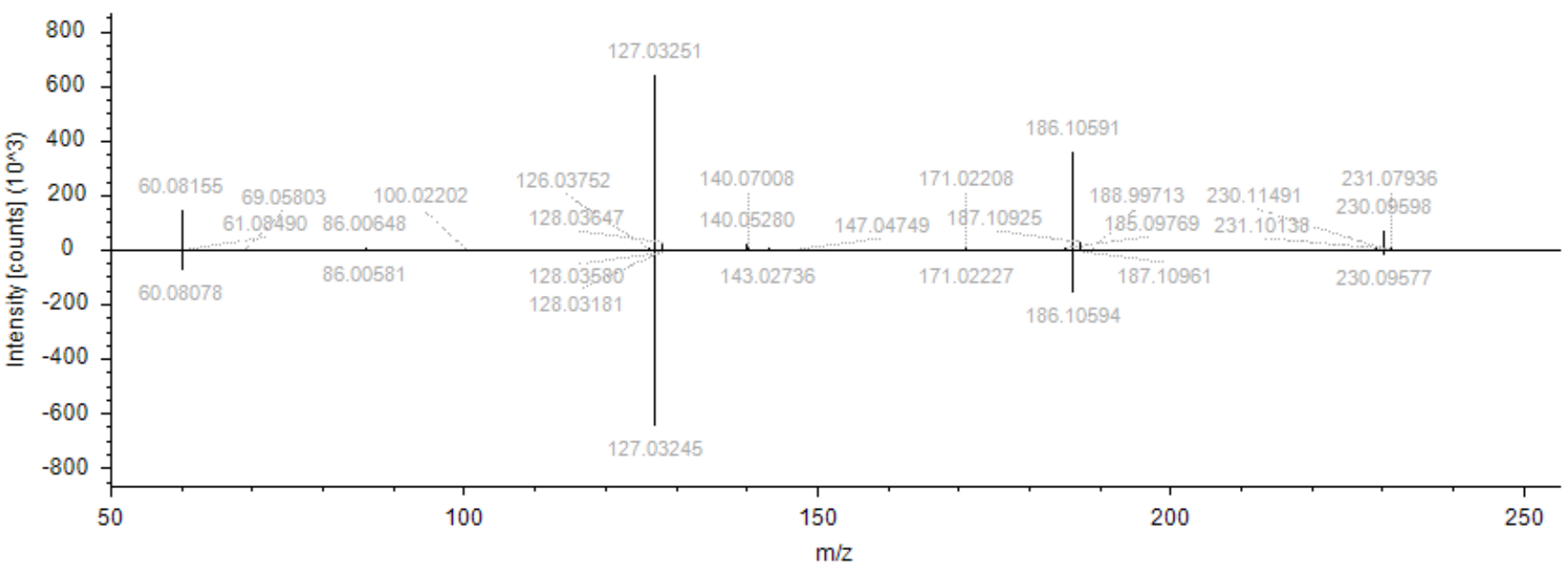

No. 43 N6,N6,N6-Trimethyl-L-lysine

RAWFILE(top): BP_0510_1 (F10) \#4032, RT=13.543 min, MS2, FTMS (+), (HCD, DDA, 189.1595@30,+1)

REFERENCE(bottom): mzCloud library, N6,N6,N6-Trimethyl-L-lysine, C9 H20 N2 O2, MS2, FTMS, (HCD, 189.1598@30)

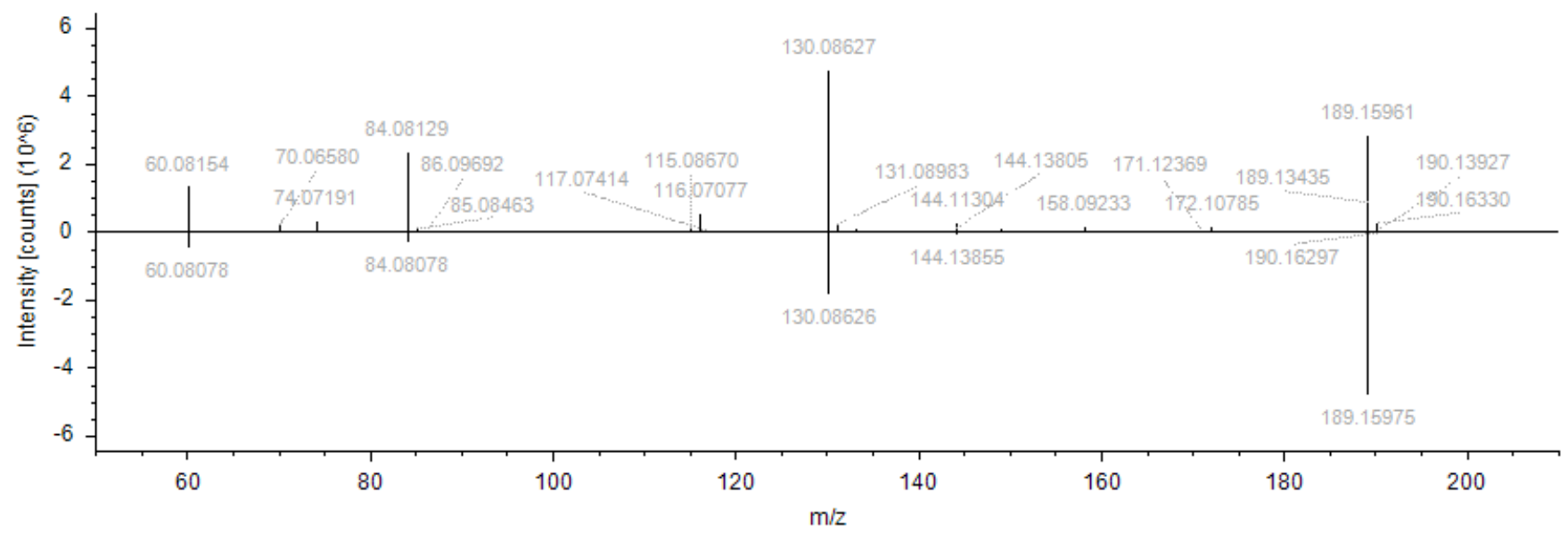


No. 47 N3,N4-Dimethyl-L-arginine

RAWFILE(top): BP_0419_2 (F2) \#3723, RT=12.556 min, MS2, FTMS (+), (HCD, DDA, 203.1500@30,+1)

REFERENCE(bottom): mzCloud library, N3,N4-Dimethyl-L-arginine, C8 H18 N4 O2, MS2, FTMS, (HCD, 203.1503@20)

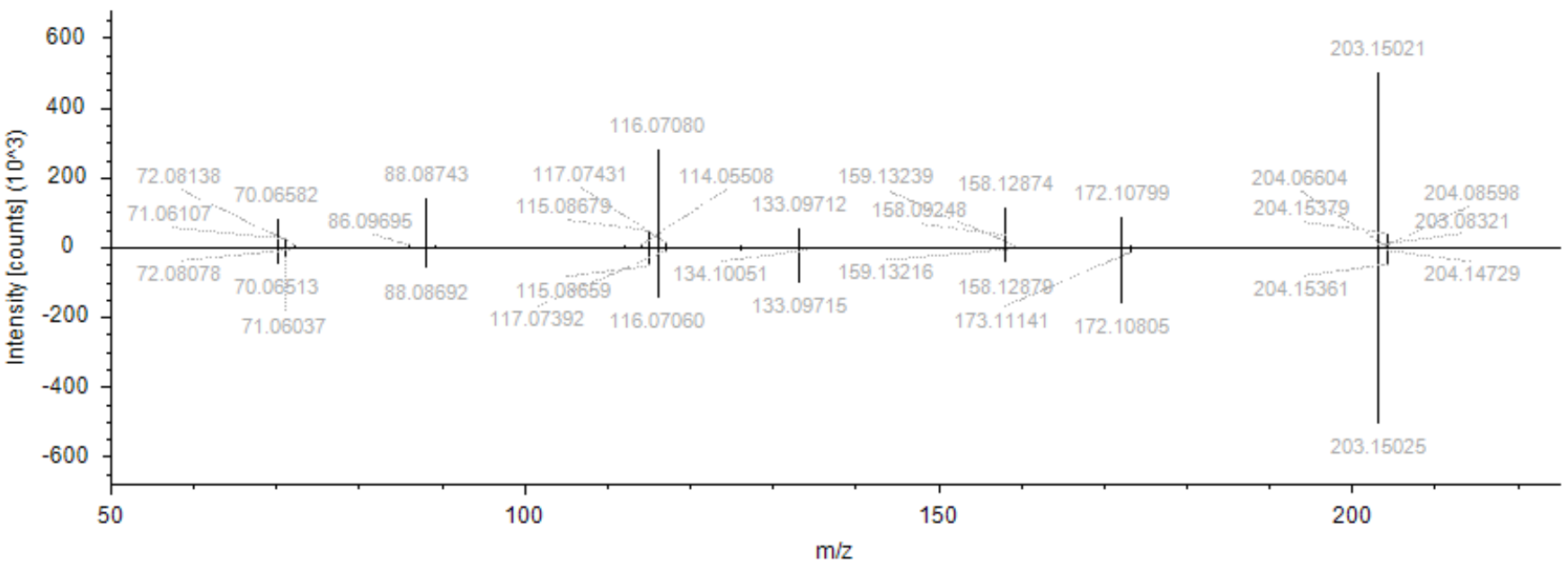

No. 48 N6-Acetyl-L-lysine

RAWFILE(top): BP_0510_3 (F12) \#2824, RT=9.441 min, MS2, FTMS (+), (HCD, DDA, 189.1233@30,+1)

REFERENCE(bottom): mzCloud library, N6-Acetyl-L-lysine, C8 H16 N2 O3, MS2, FTMS, (HCD, 189.1234@30)

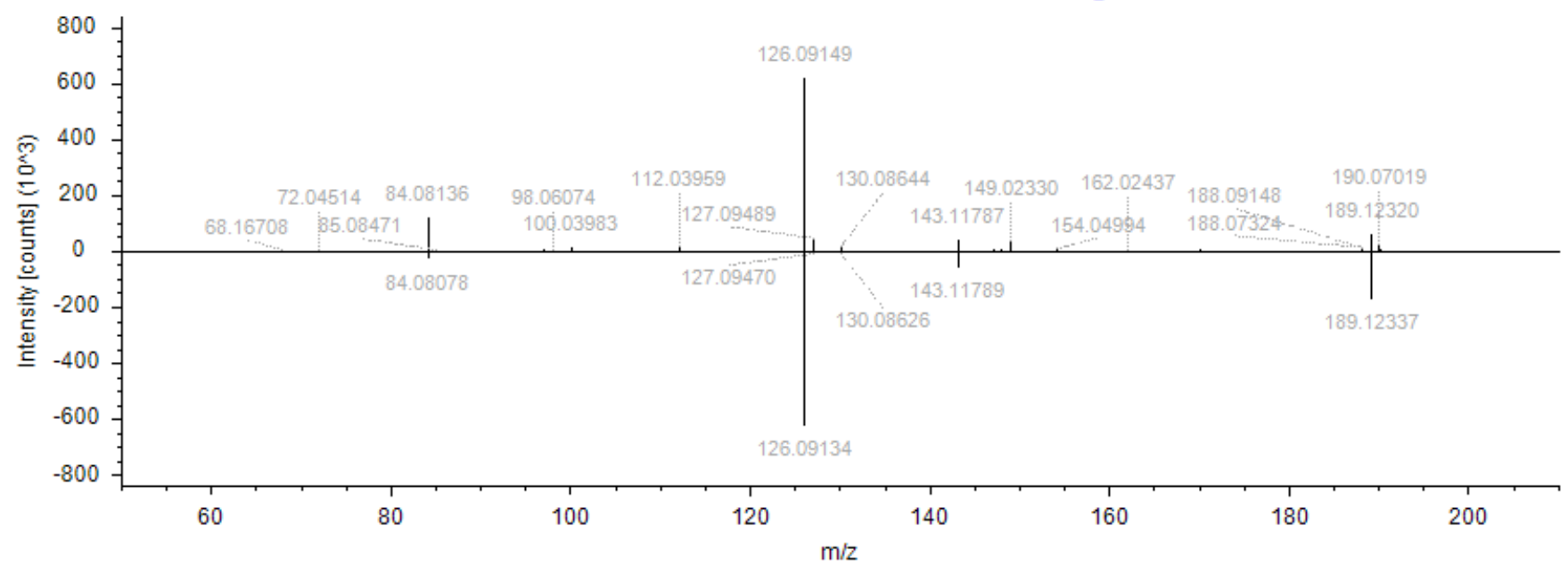

No. 49 L-Saccharopine

RAWFILE(top): J3_0419_2 (F4) \#4672, RT=15.606 min, MS2, FTMS (+), (HCD, DDA, 277.1391@30, +1)

REFERENCE(bottom): mzCloud library, L-Saccharopine, C11 H20 N2 O6, MS2, FTMS, (HCD, 277.1394@30)

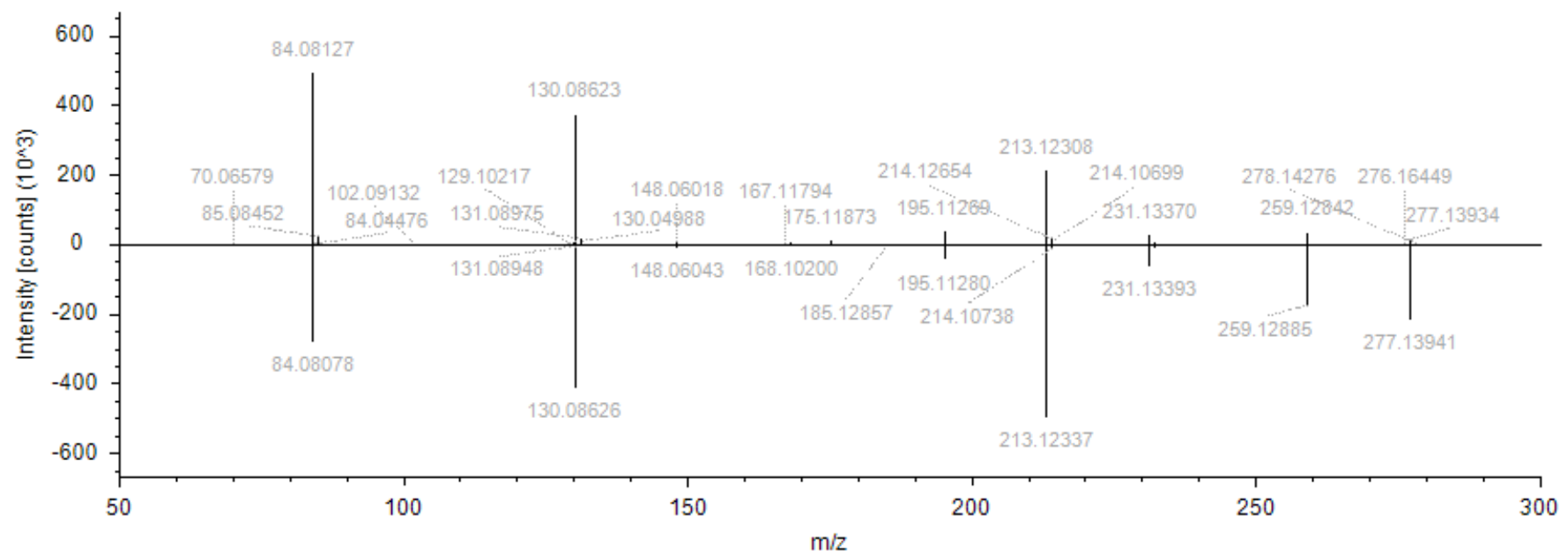


No. 50 Argininosuccinic acid

RAWFILE(top): BP_0419_3 (F3) \#4817, RT=16.088 min, MS2, FTMS (+), (HCD, DDA, 291.1295@30, +1)

REFERENCE(bottom): mzCloud library, Argininosuccinic acid, C10 H18 N4 O6, MS2, FTMS, (HCD, 291.1299@30)

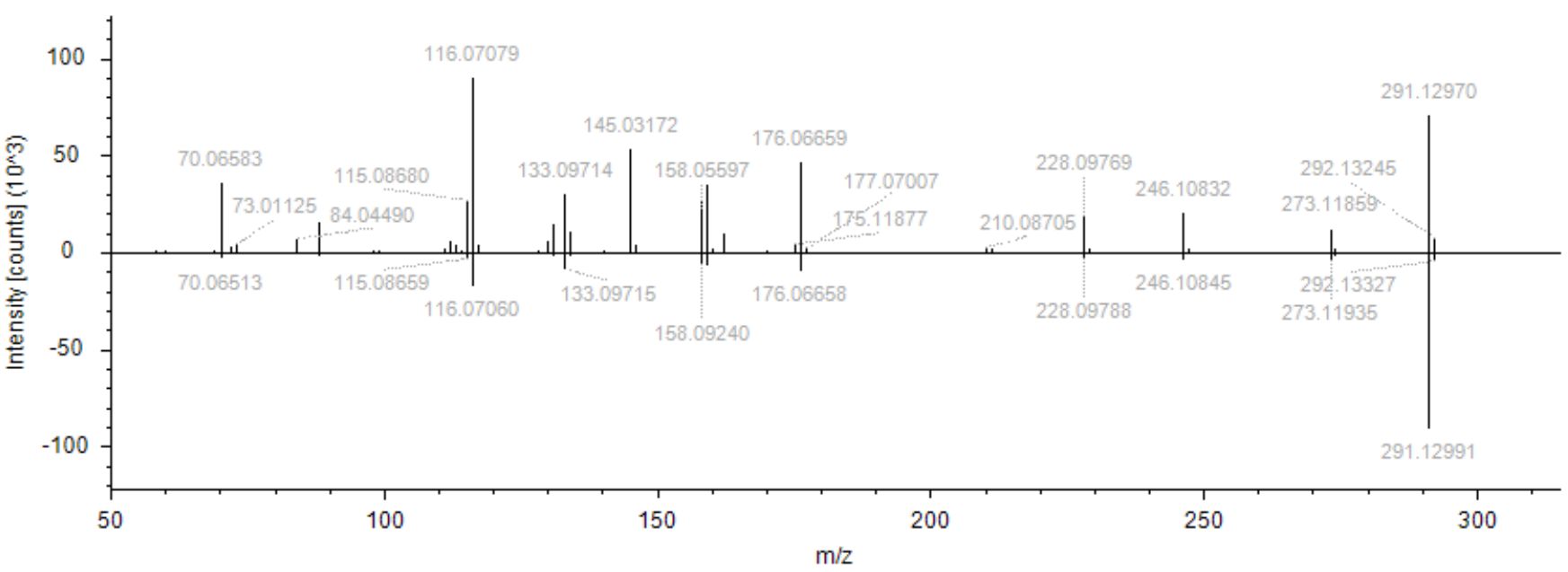

No. $51 \gamma$-Glutamylcysteine

RAWFILE(top): BP_0523_3 (F18) \#2531, RT=8.494 min, MS2, FTMS (+), (HCD, DDA, 251.0697@30, +1)

REFERENCE(bottom): mzCloud library, Y-Glutamylcysteine, C8 H14 N2 O5 S, MS2, FTMS, (HCD, 251.0696@30)

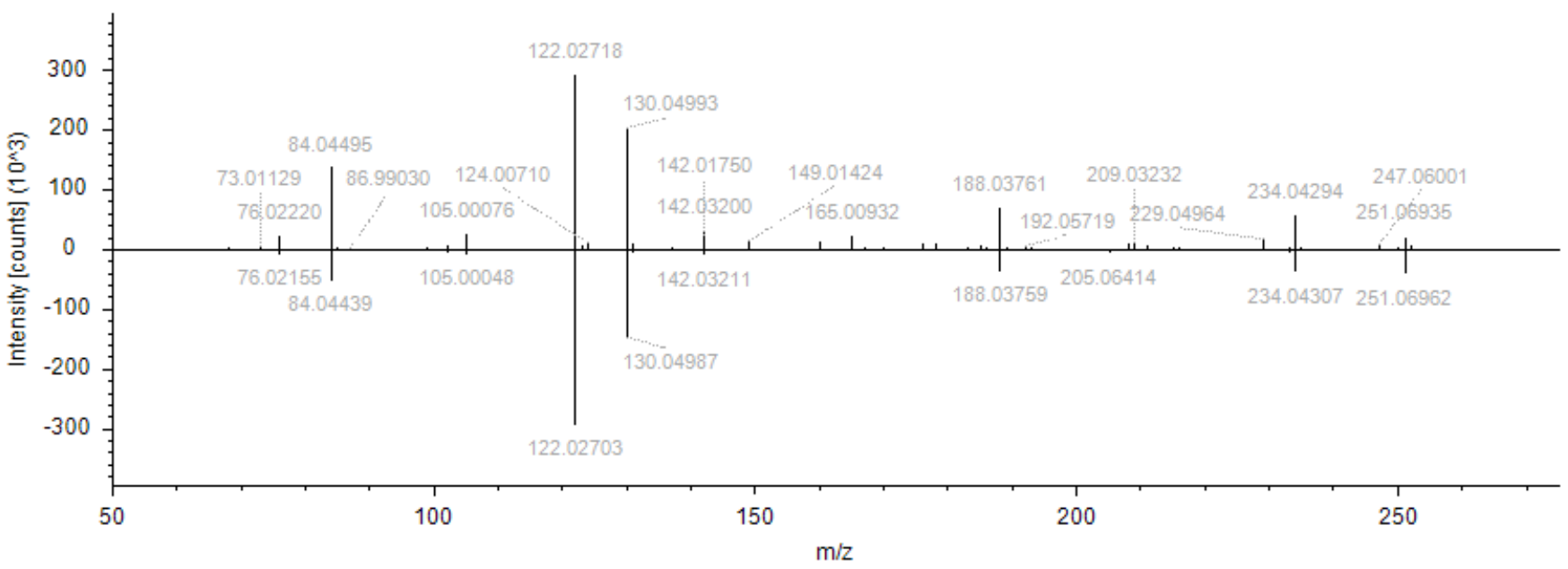

No. 52 1-Methylhistidine

RAWFILE(top): YA-0418-3 (F6) \#1853, RT=5.901 min, MS2, FTMS (+), (HCD, DDA, 170.0921@30, +1)

REFERENCE(bottom): mzCloud library, Methyl-L-histidinate, C7 H11 N3 O2, MS2, FTMS, (HCD, 170.0924@30)

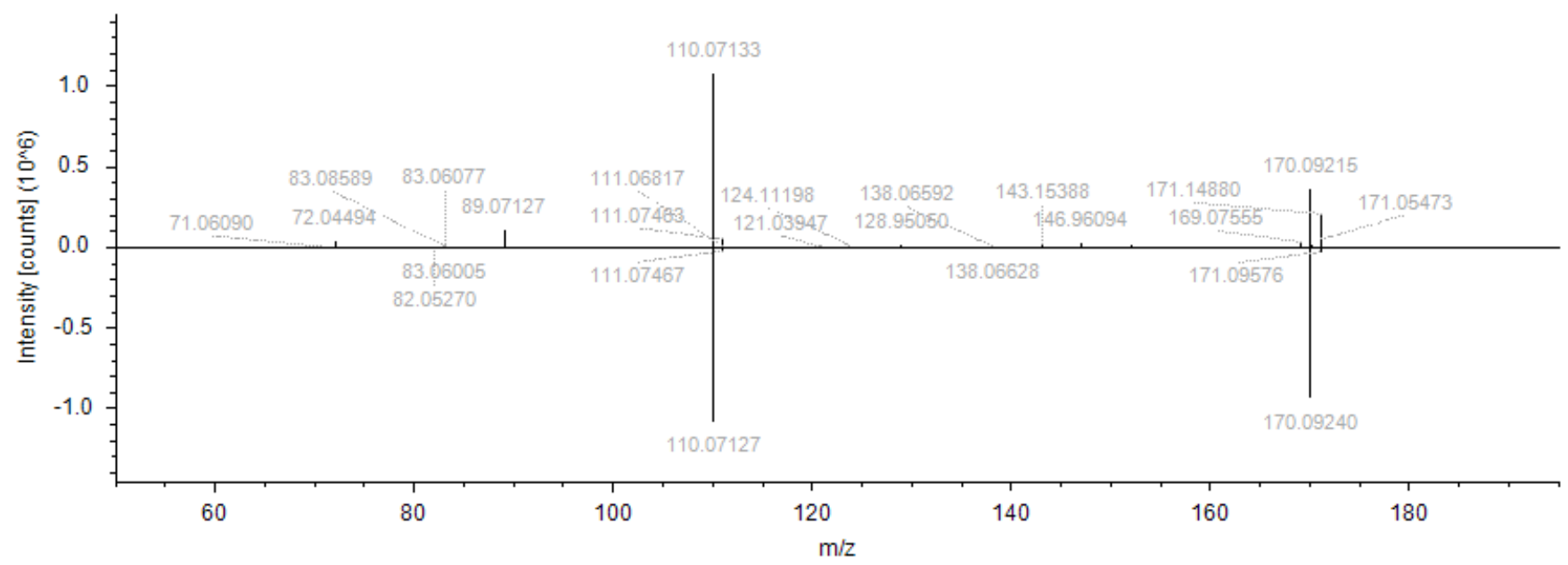


No. 59 S-Adenosylmethionine

RAWFILE(top): BP 0419 1 (F1) \#4708, RT=15.662 min, MS2, FTMS (+), (HCD, DDA, 399.1443@30,+1)

REFERENCE(bottom): mżCloud library, S-Adenosylmethionine, C15 H22 N6 O5 S, MS2, FTMS, (HCD, 399.1445@30)

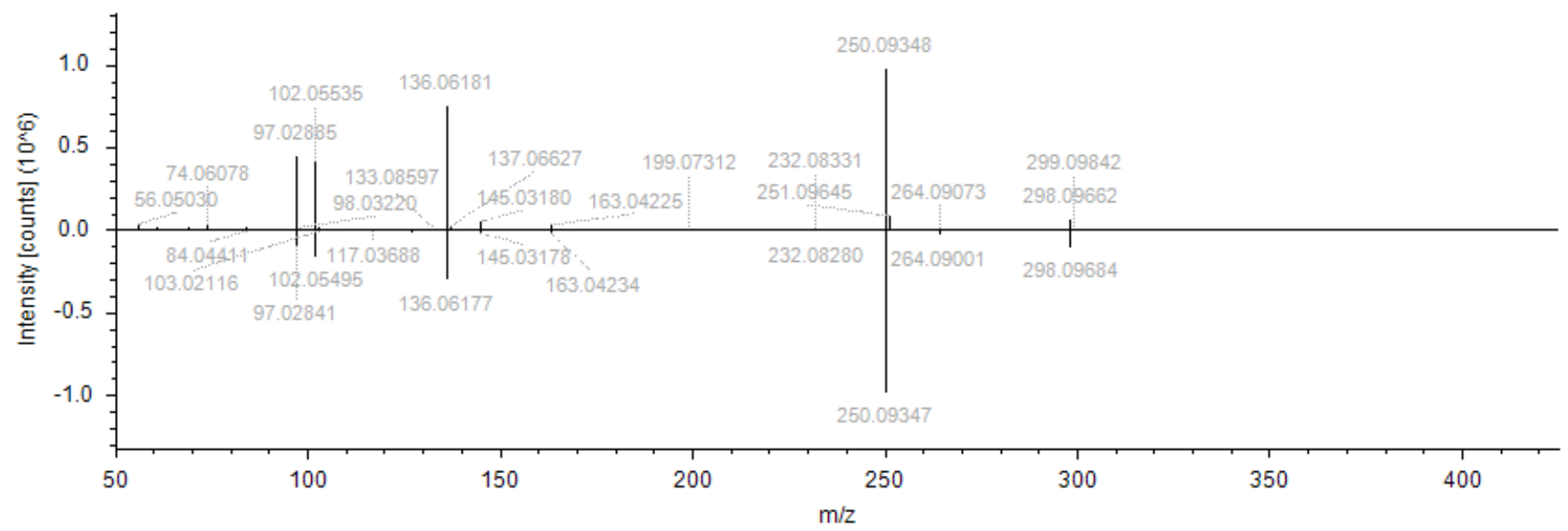

\section{No. 60 Adenosine}

RAWFILE(top): BP_0419_3 (F3) \#824, RT=2.765 min, MS2, FTMS (+), (HCD, DDA, 268.1037@30,+1) REFERENCE(bottom): mzCloud library, Adenosine, C10 H13 N5 04, MS2, FTMS, (HCD, 268.1040@30)

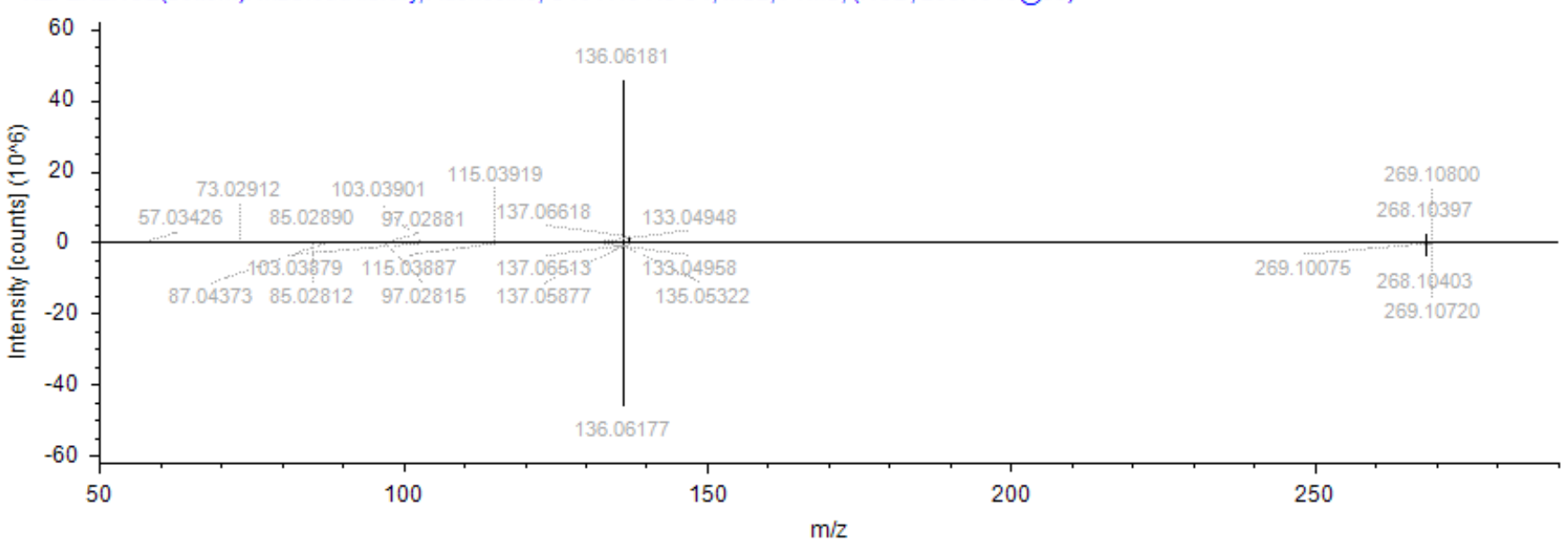

No. 61 2'-Deoxyadenosine

RAWFILE(top): BP_0419_3 (F3) \#692, RT=2.300 min, MS2, FTMS (+), (HCD, DDA, 252.1087@30,+1)

REFERENCE(bottom): mzCloud library, 2'-Deoxyadenosine, C10 H13 N5 O3, MS2, FTMS, (HCD, 252.1091@30)

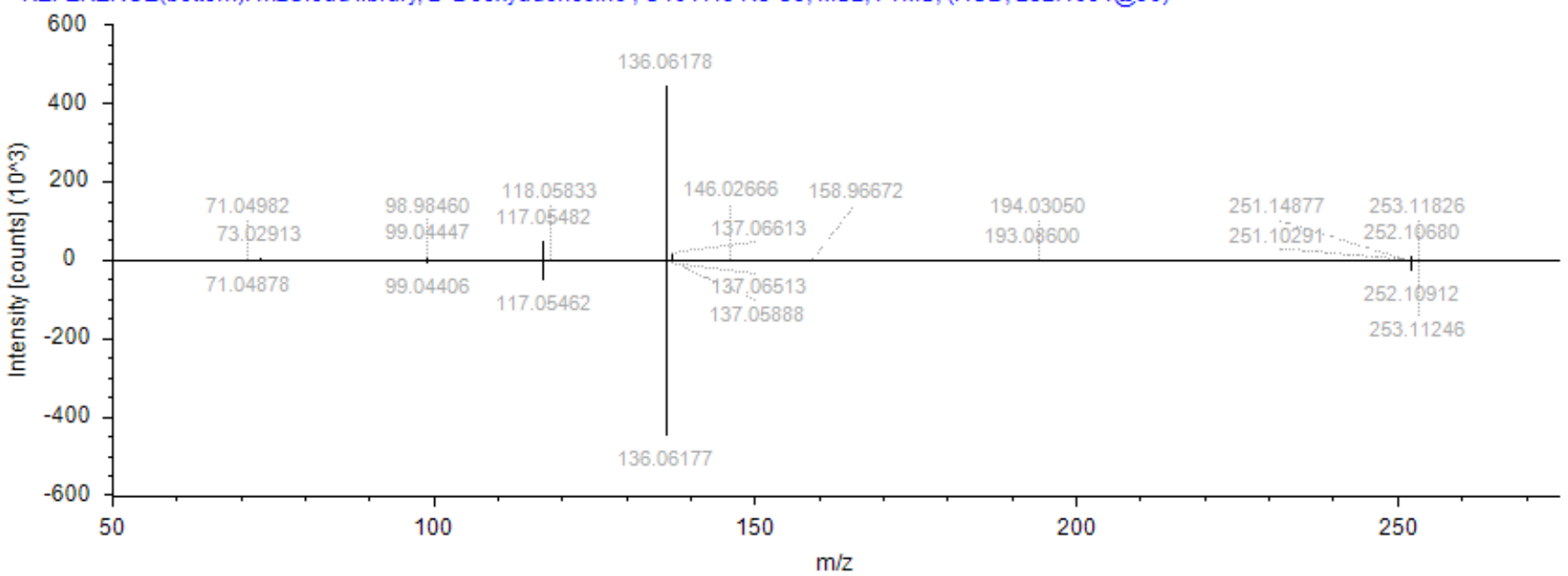


No. 62 3-Methyladenine

RAWFILE(top): BP_0523_2 (F17) \#1316, RT=4.411 min, MS2, FTMS (+), (HCD, DDA, 150.0772@30,+1)

REFERENCE(bottom): mzCloud library, N6-Methyladenine, C6 H7 N5, MS2, FTMS, (HCD, 150.0774@10)

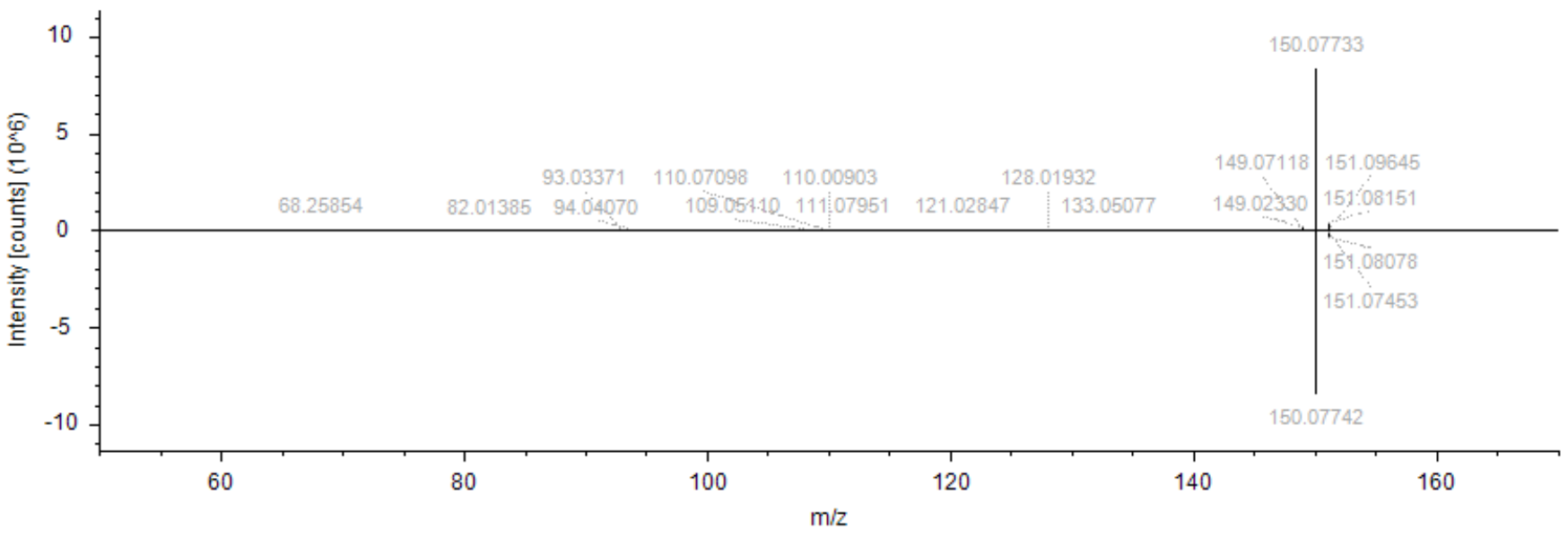

No. 63 Adenine

RAWFILE(top): BP_0419_3 (F3) \#758, RT=2.536 min, MS2, FTMS (+), (HCD, DDA, 136.0617@30, +1)

REFERENCE(bottom): mzCloud library, Adenine, C5 H5 N5, MS2, FTMS, (HCD, 136.0618@50)

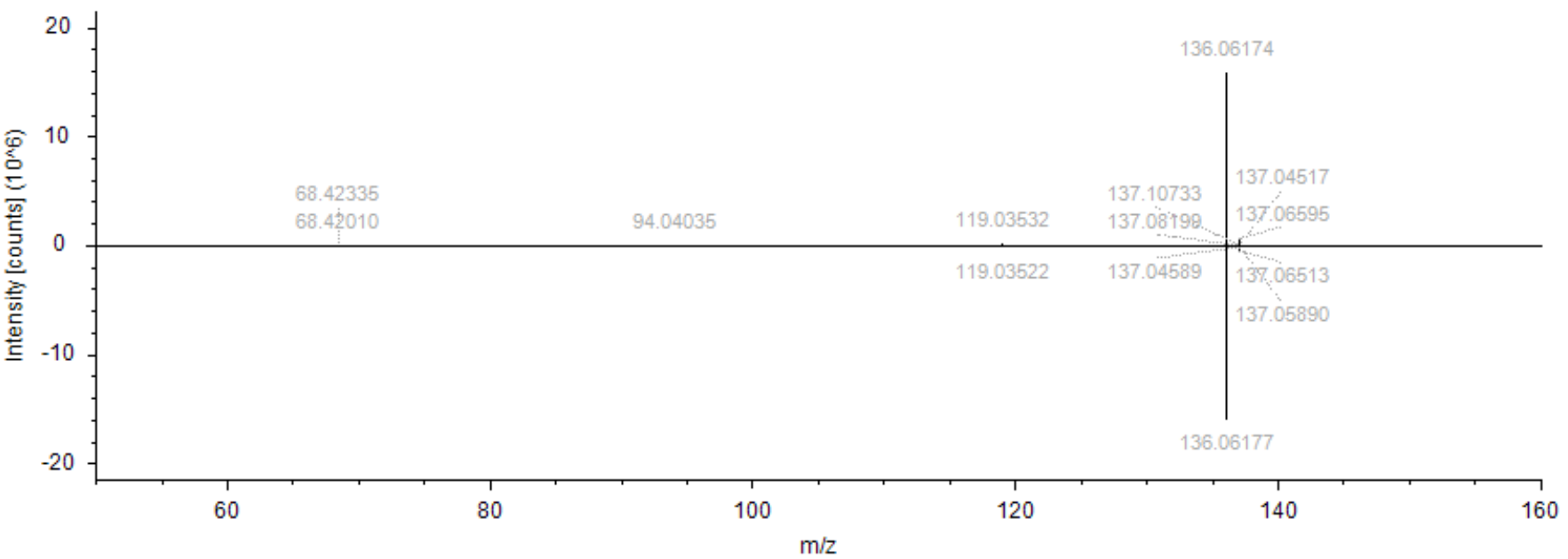

\section{No. 64 Uridine}

RAWFILE(top): BP_0419_3 (F3) \#938, RT=2.883 min, MS2, FTMS (-), (HCD, DDA, 243.0619@30, -1) REFERENCE(bottom): mzCloud library, Uridine, C9 H12 N2 O6, MS2, FTMS, (HCD, 243.0623@40)

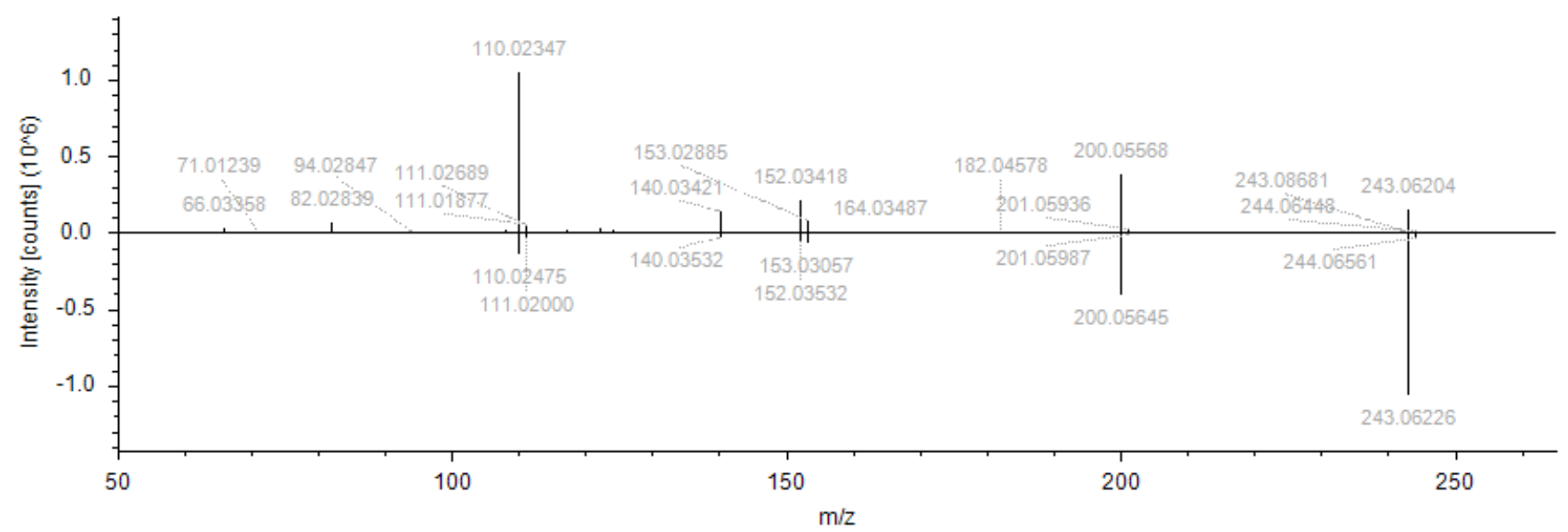




\section{No. 65 Guanosine}

RAWFILE(top): BP_0419_3 (F3) \#1906, RT=5.847 min, MS2, FTMS (-), (HCD, DDA, 282.0842@30, -1)

REFERENCE(bottom): mzCloud library, Guanosine, C10 H13 N5 O5, MS2, FTMS, (HCD, 282.0844@30)

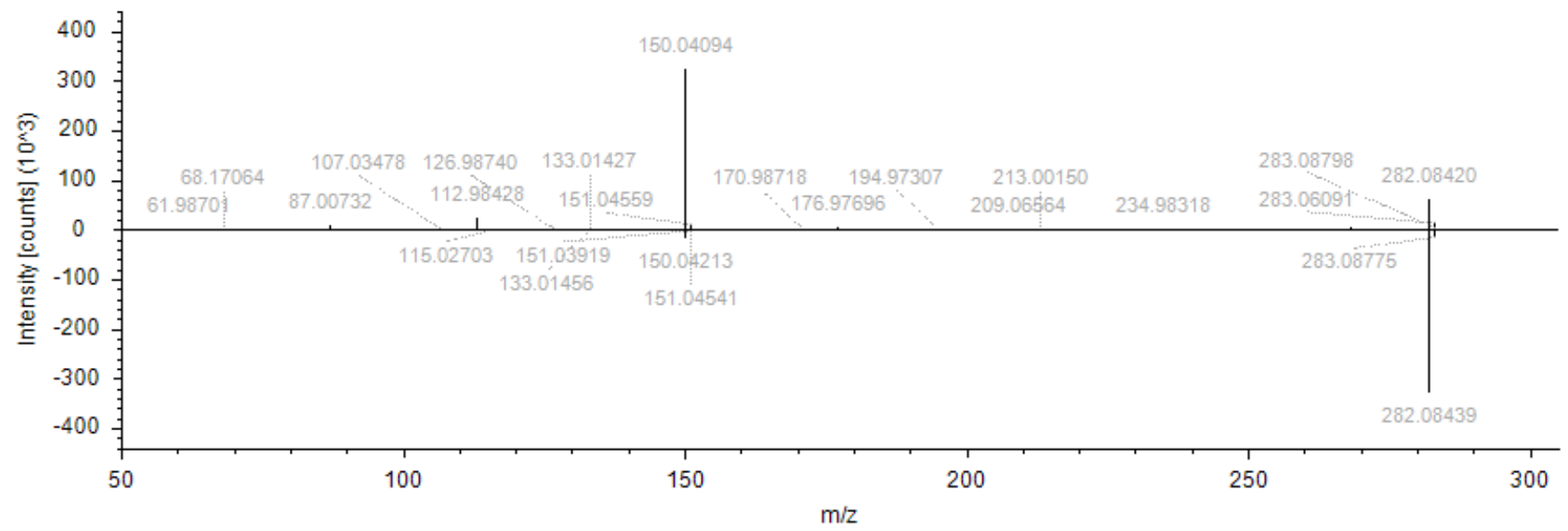

\section{No. 66 5'-S-Methyl-5'-thioadenosine}

RAWFILE(top): BP_0419_1 (F1) \#518, RT=1.618 min, MS2, FTMS (+), (HCD, DDA, 298.0962@30,+1)

REFERENCE(bottom): mzCloud library, 5'-S-Methyl-5'-thioadenosine, C11 H15 N5 O3 S, MS2, FTMS, (HCD, 298.0968@30)

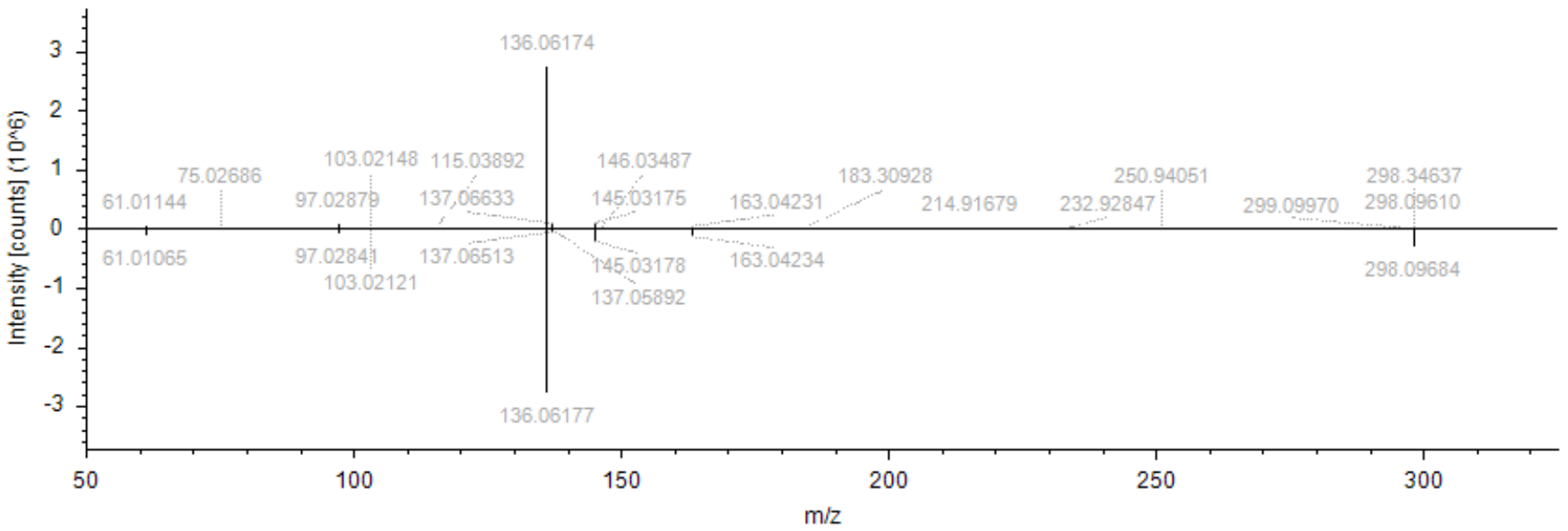

No. 68 Uridine 5'-diphosphogalactose

RAWFILE(top): 0621-3 (F5) \#5103, RT=17.177 min, MS2, FTMS (-), (HCD, DDA, 565.0474@30, -1)

REFERENCE(bottom): mzCloud library, Uridine 5'-diphosphogalactose, C15 H24 N2 O17 P2, MS2, FTMS, (HCD, 565.0477@50)

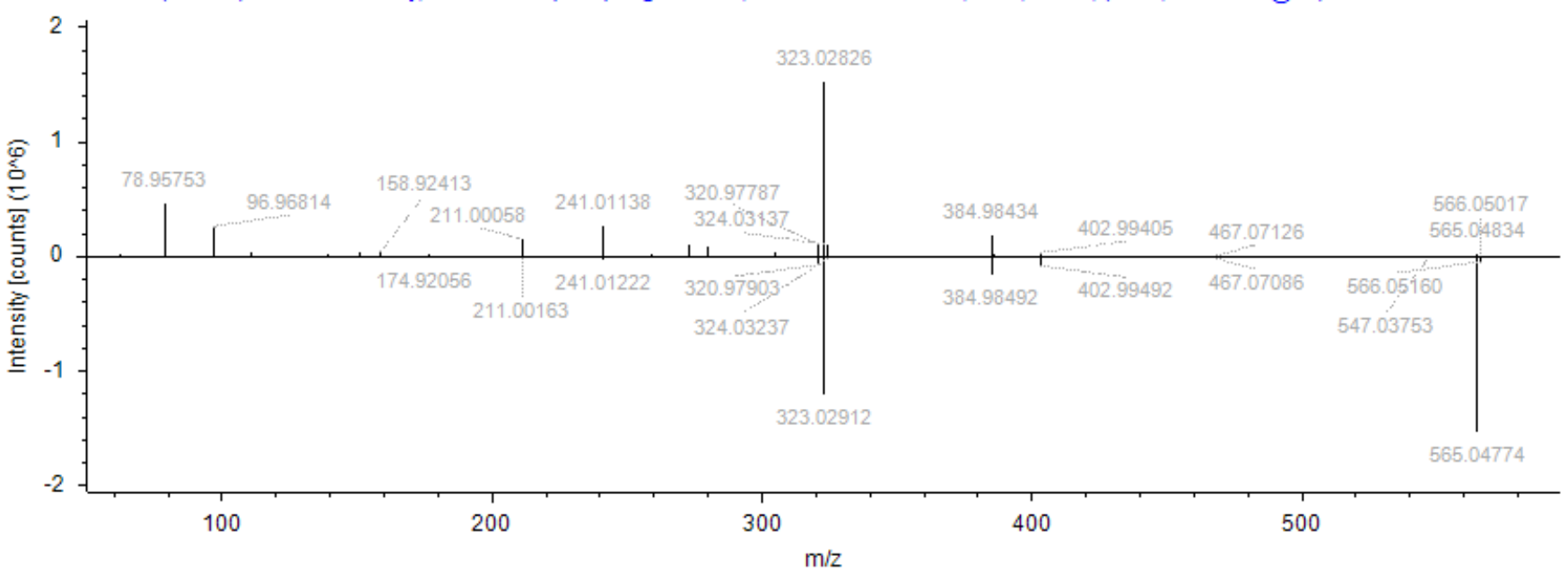




\section{No. 70 Pseudouridine}

RAWFILE(top): QC-MS2-2 (F12) \#2311, RT=4.654 min, MS2, FTMS (-), (HCD, DDA, 242.0516@30, -1)

REFERENCE(bottom): mzCloud library, Pseudouridine, C9 H12 N2 O6, MS2, FTMS, (HCD, 243.0623@40)

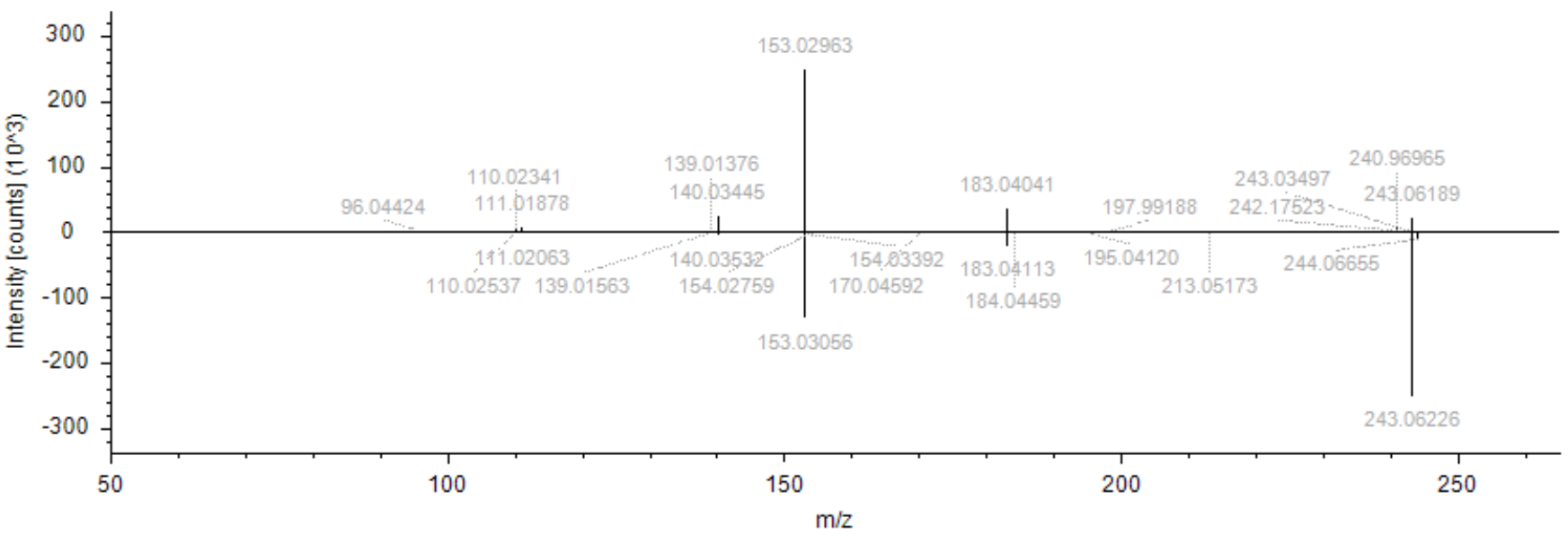

No. 71 7-Methylguanosine

RAWFILE(top): 0719-1 (F16)\#1200, RT=4.096 min, MS2, FTMS (+), (HCD, DDA, 298.1140@30,+1)

REFERENCE(bottom): mzCloud library, 7-Methylguanosine, C11 H15 N5 O5, MS2, FTMS, (HCD, 298.1146@30)

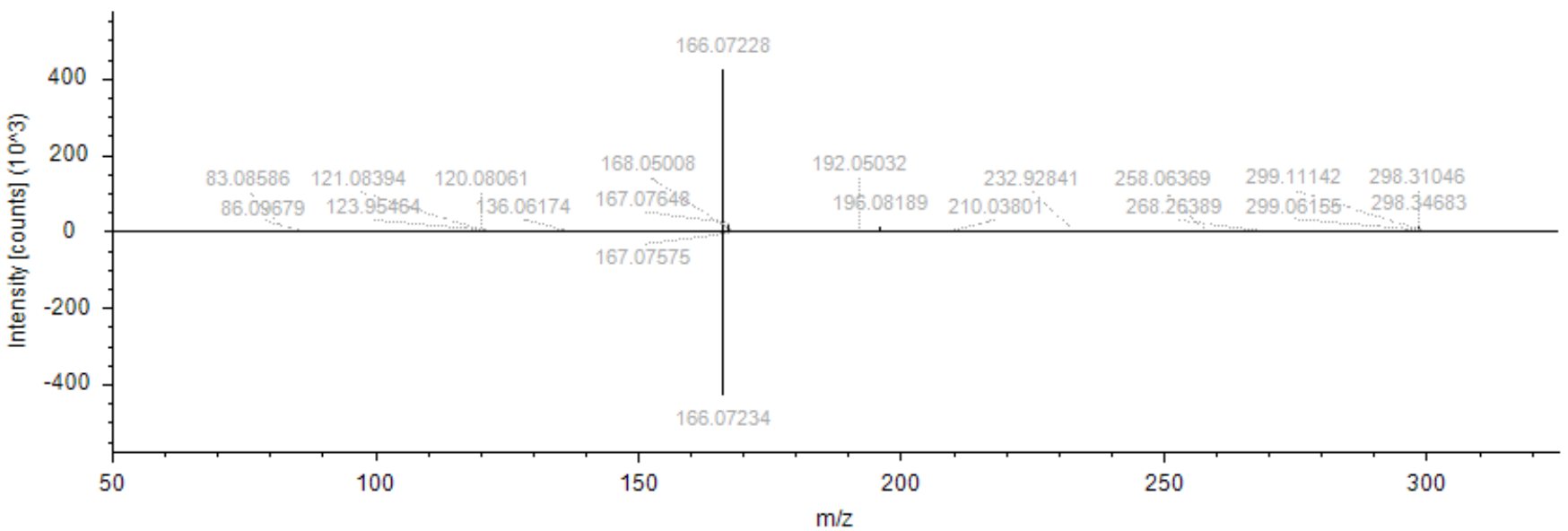

No. 72 Cytosine

RAWFILE(top): BP_0523_2 (F17) \#1420, RT=4.775 min, MS2, FTMS (+), (HCD, DDA, 112.0506@30, +1)

REFERENCE(bottom): mzCloud library, Cytosine, C4 H5 N3 O, MS2, FTMS, (HCD, 112.0505@40)

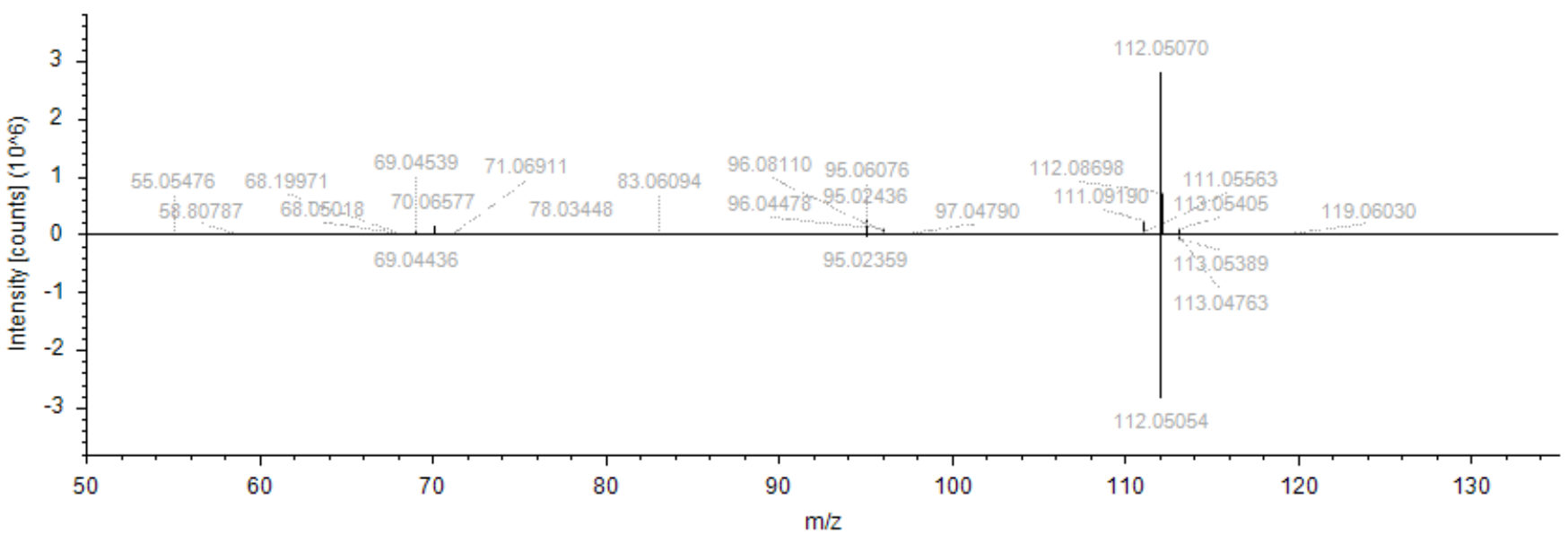


No. 76 Acetylcholine

RAWFILE(top): BP_0425_2 (F5) \#1100, RT=3.671 min, MS2, FTMS (+), (HCD, DDA, 146.1173@30,+1)

REFERENCE(bottom): mzCloud library, Acetylcholine, C7 H15 N O2, MS2, FTMS, (HCD, 146.1176@30)

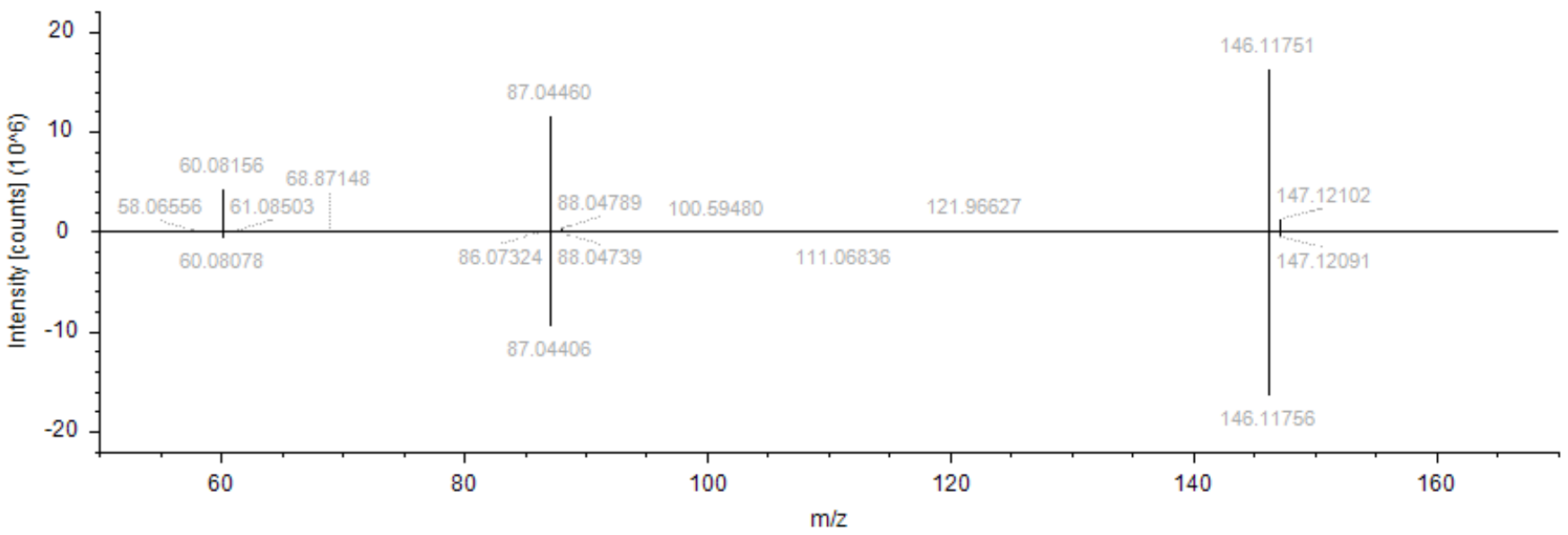

No. 79 Stachydrine

RAWFILE(top): Jl-0530-2 (F24) \#1683, RT=5.501 min, MS2, FTMS (+), (HCD, DDA, 144.0803@30, +1) REFERENCE(bottom): mzCloud library, DL-Stachydrine, C7 H13 N O2, MS2, FTMS, (HCD, 144.1019@50)

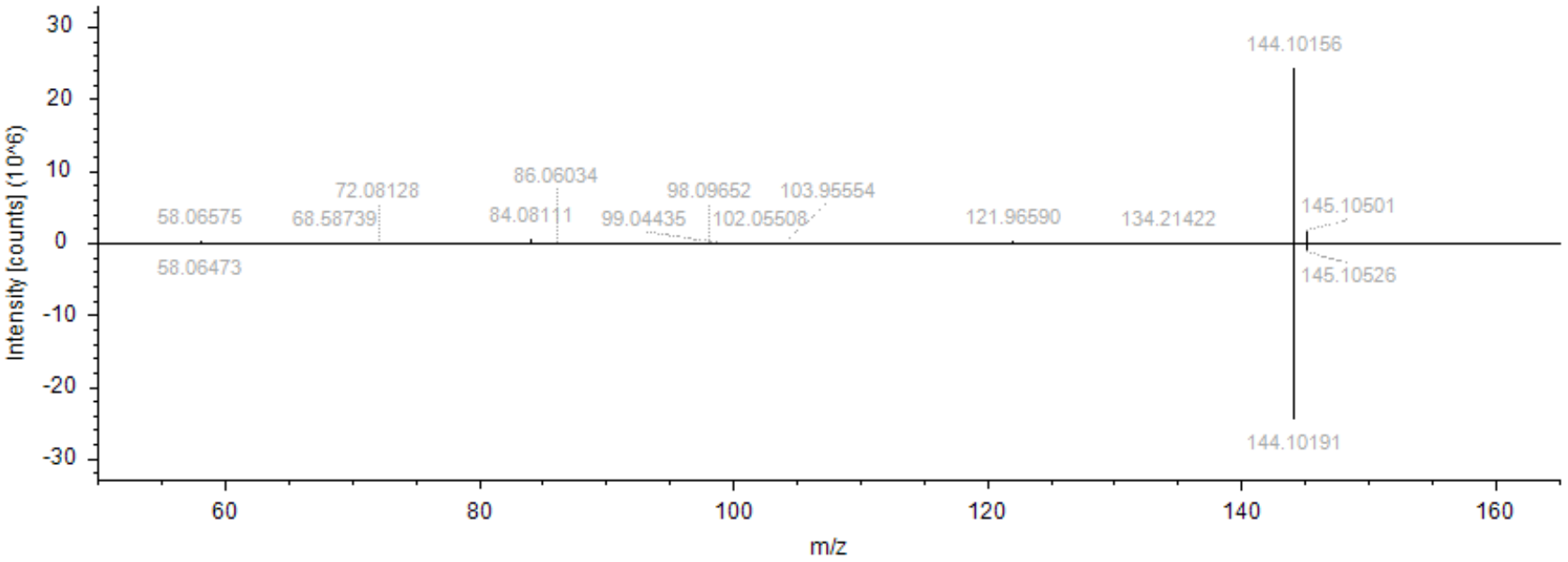

No. 80 D-Raffinose

RAWFILE(top): BP_0419_3 (F3) \#3920, RT=13.182 min, MS2, FTMS (+), (HCD, DDA, 527.1580@30, +1) REFERENCE(bottom): mzCloud library, D-Raffinose, C18 H32 016, MS2, FTMS, (HCD, 527.1583@40)

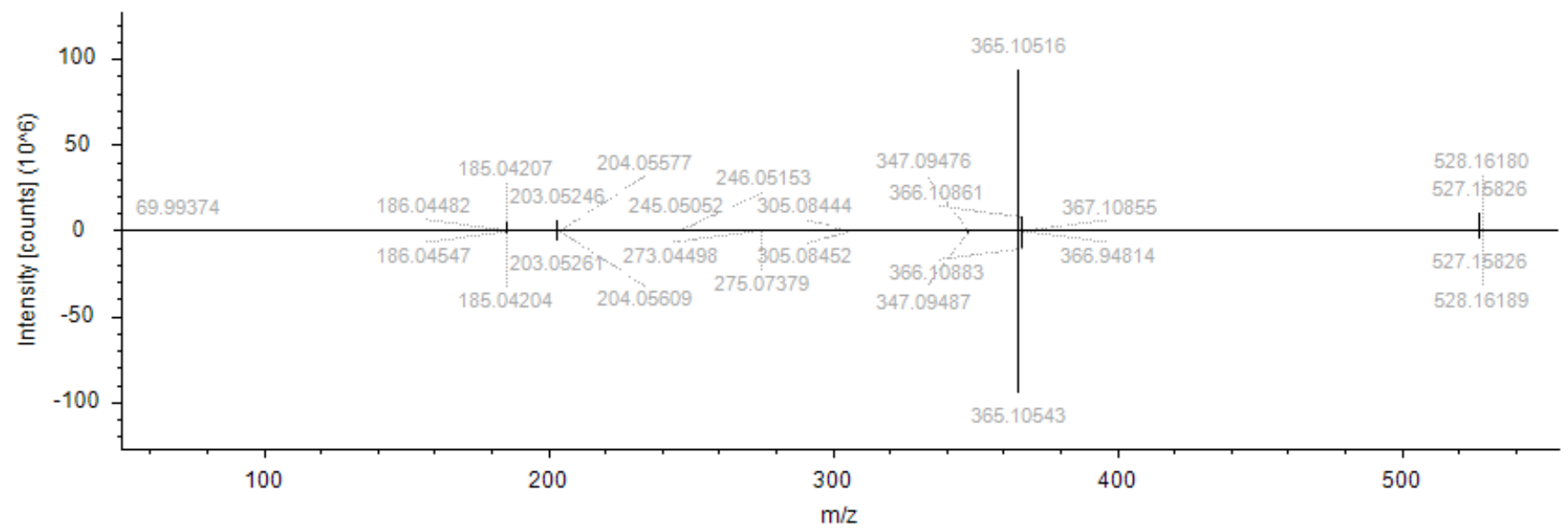




\section{No. 82 Dulcitol}

RAWFILE(top): BP_0419_1 (F1) \#1994, RT=6.307 min, MS2, FTMS (-), (HCD, DDA, 181.0710@30, -1)

REFERENCE(bottom): mżCloud library, Dulcitol, C6 H14 O6, MS2, FTMS, (HCD, 181.0718@40)

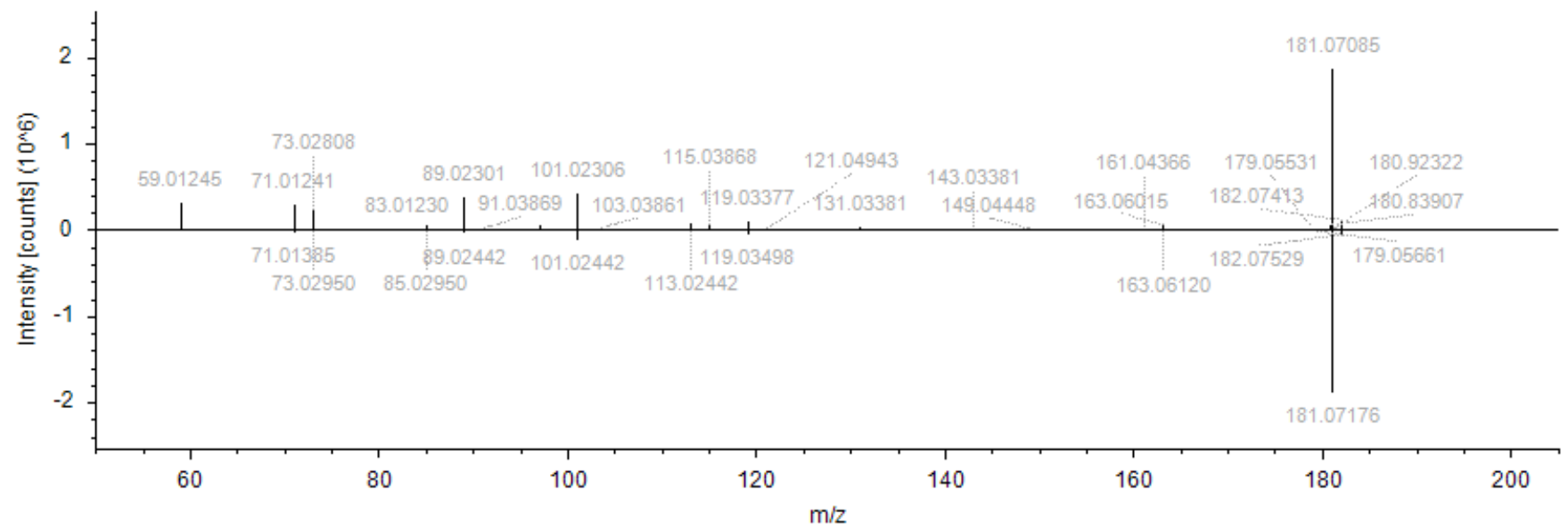

No. 84 Nicotinamide

RAWFILE(top): BP_0510_3 (F12) \#2054, RT=6.935 min, MS2, FTMS (+), (HCD, DDA, 123.0554@30, +1)

REFERENCE(bottom): mzCloud library, Nicotinamide, C6 H6 N2 O, MS2, FTMS, (HCD, 123.0553@40)

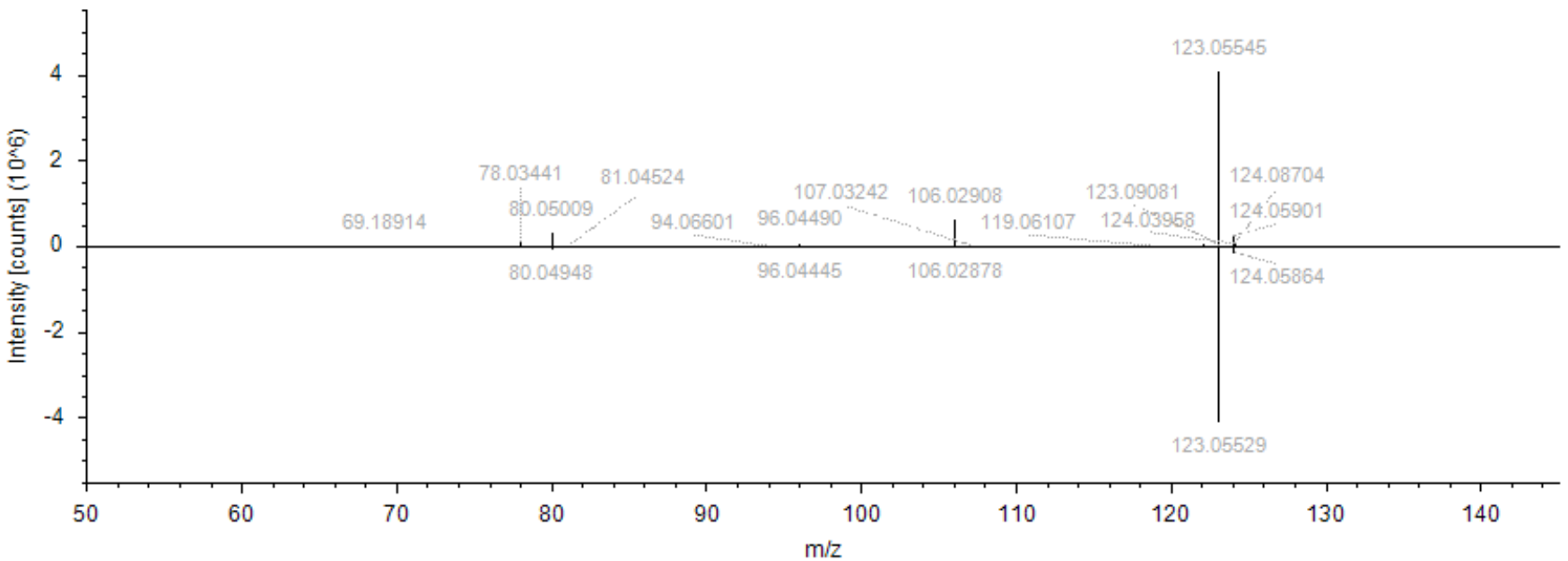

No. 85 Pantothenic acid

RAWFILE(top): J4_0523_1 (F18) \#495, RT=1.391 min, MS2, FTMS (-), (HCD, DDA, 218.1026@30, -1)

REFERENCE(bottom): mzCloud library, Pantothenic acid, C9 H17 N O5, MS2, FTMS, (HCD, 218.1034@50)

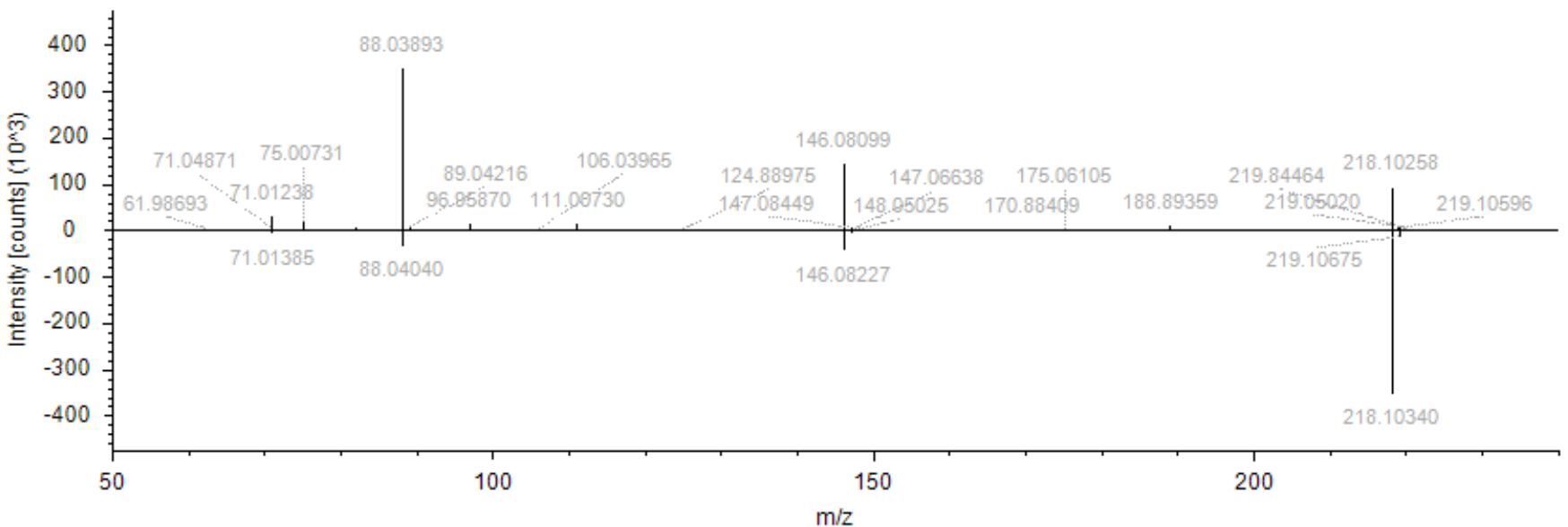




\section{No. 86 Pyridoxine}

RAWFILE(top): BP_0419_3 (F3) \#752, RT=2.513 min, MS2, FTMS (+), (HCD, DDA, 170.0809@30, +1)

REFERENCE(bottom): mzCloud library, Pyridoxine, C8 H11 N O3, MS2, FTMS, (HCD, 170.0812@30)

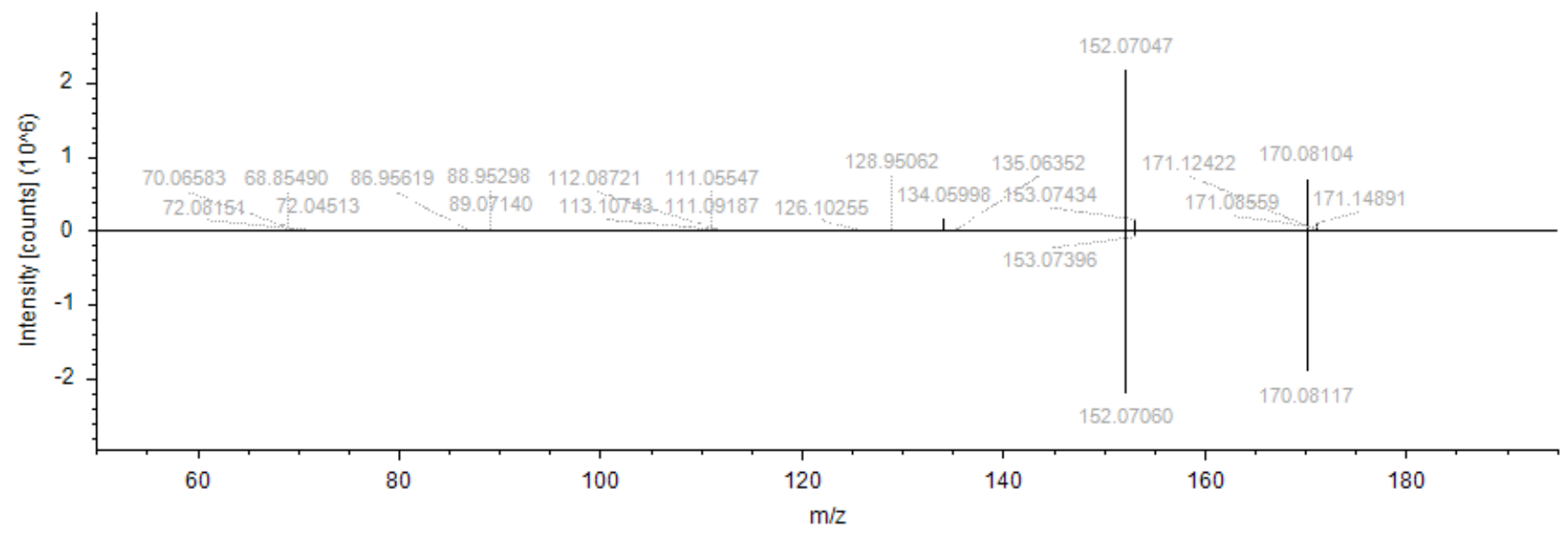

No. 87 Phytosphingosine

RAWFILE(top): BP_0419_1 (F1) \#740, RT=2.430 min, MS2, FTMS (+), (HCD, DDA, 318.2998@30, +1)

REFERENCE(bottom): mżCloud library, 2-Amino-1,3,4-octadecanetriol, C18 H39 N O3, MS2, FTMS, (HCD, 318.3003@30)

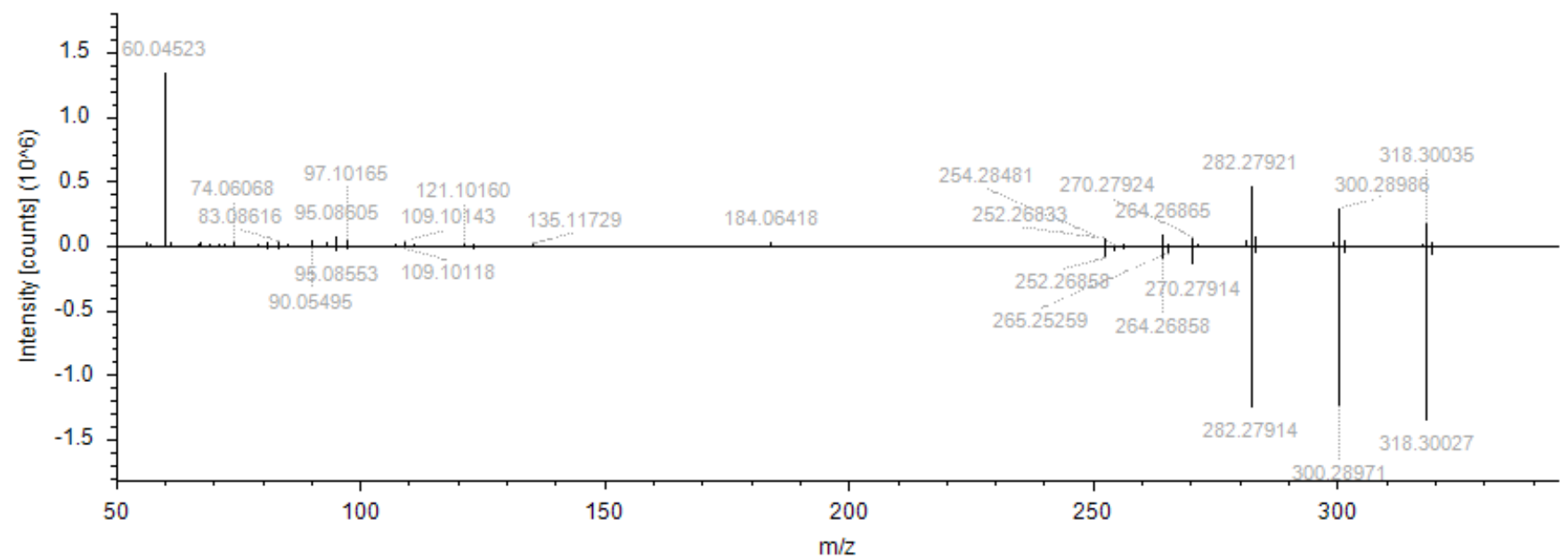

Figure S2 MS/MS spectrums of 29 compounds that identified in garlic sample (upper panel) and mzCloud (lower panel) 
No. $3 \gamma$-L-Glutamyl-S-(trans-1-propenyl)-L-cysteine

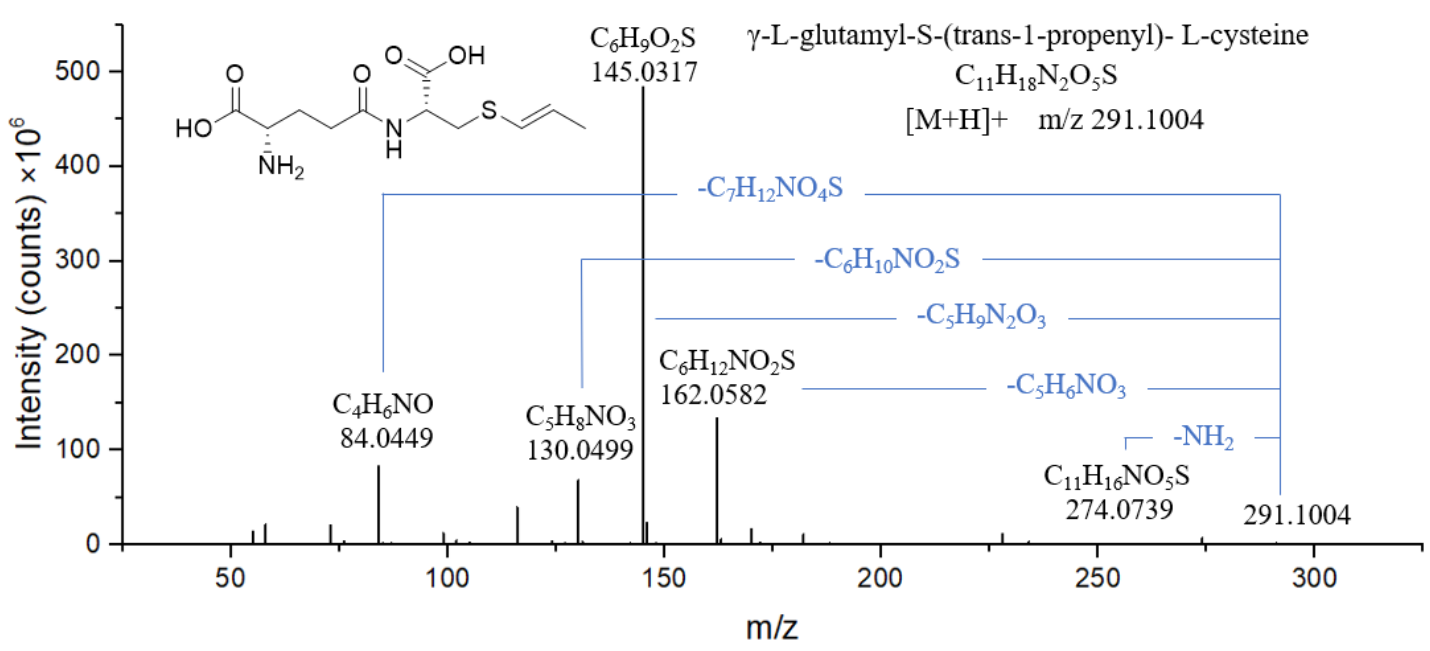

No. $4 \gamma$-Glutamyl-S-allylthio-L-cysteine

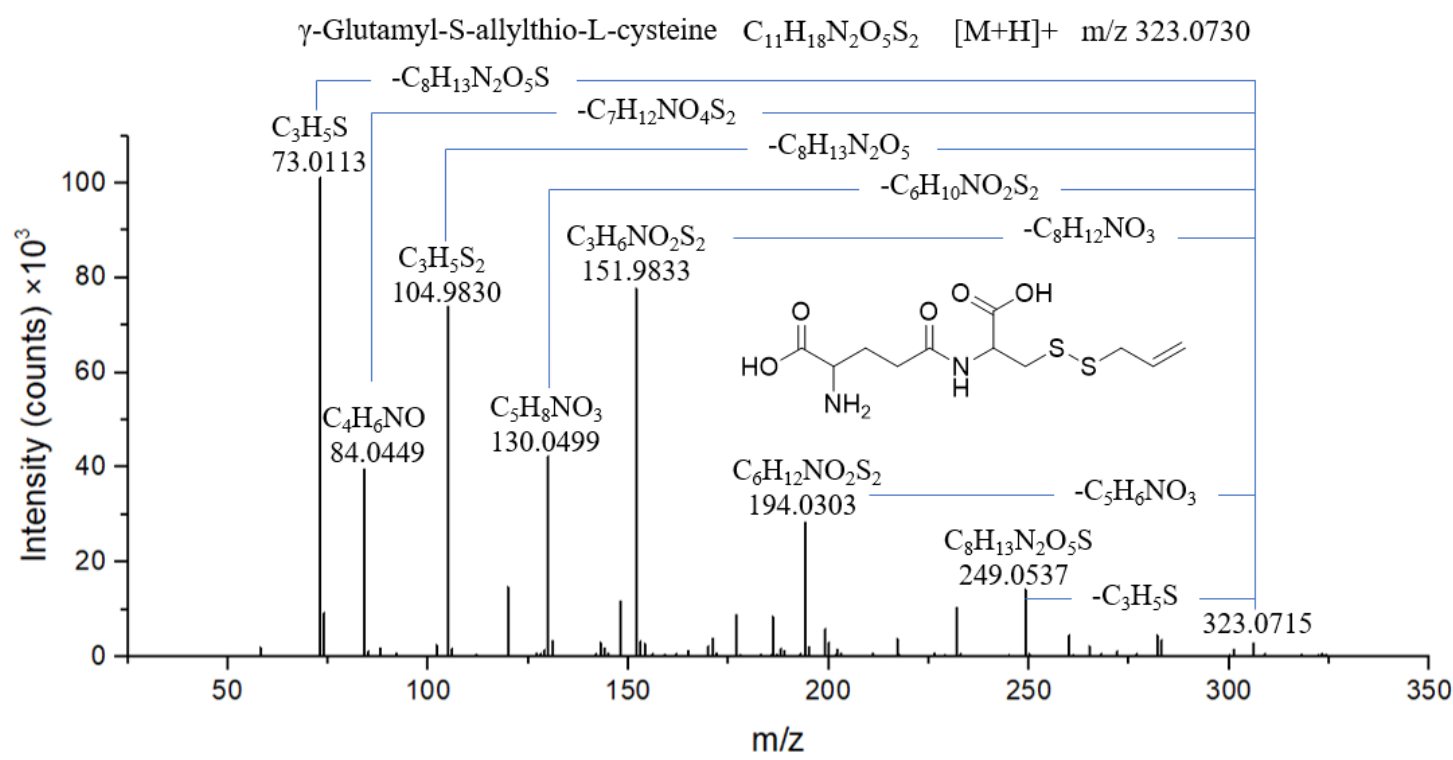

No. $5 \gamma$-Glutamyl-S-(1-propenyl)-L-cysteine sulfoxide

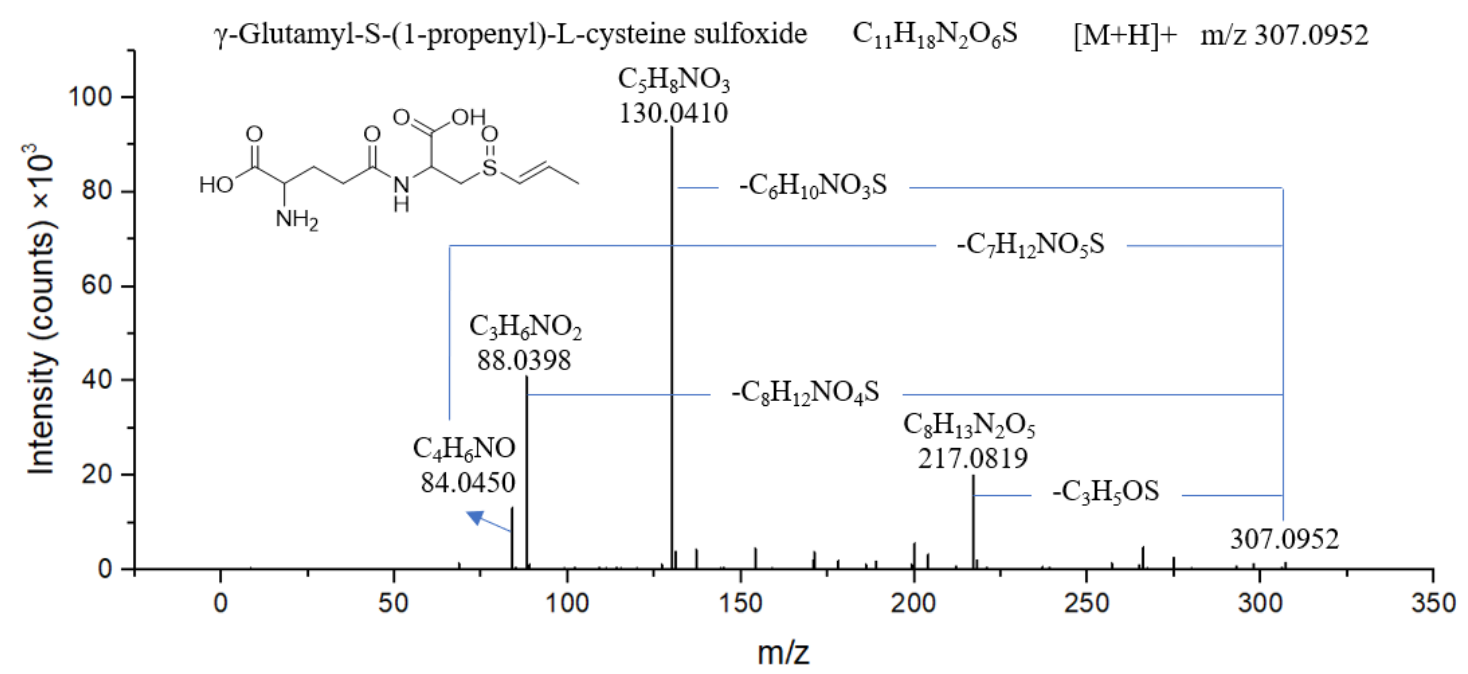


No. 9 N-2-Propyn-1-ylcysteine

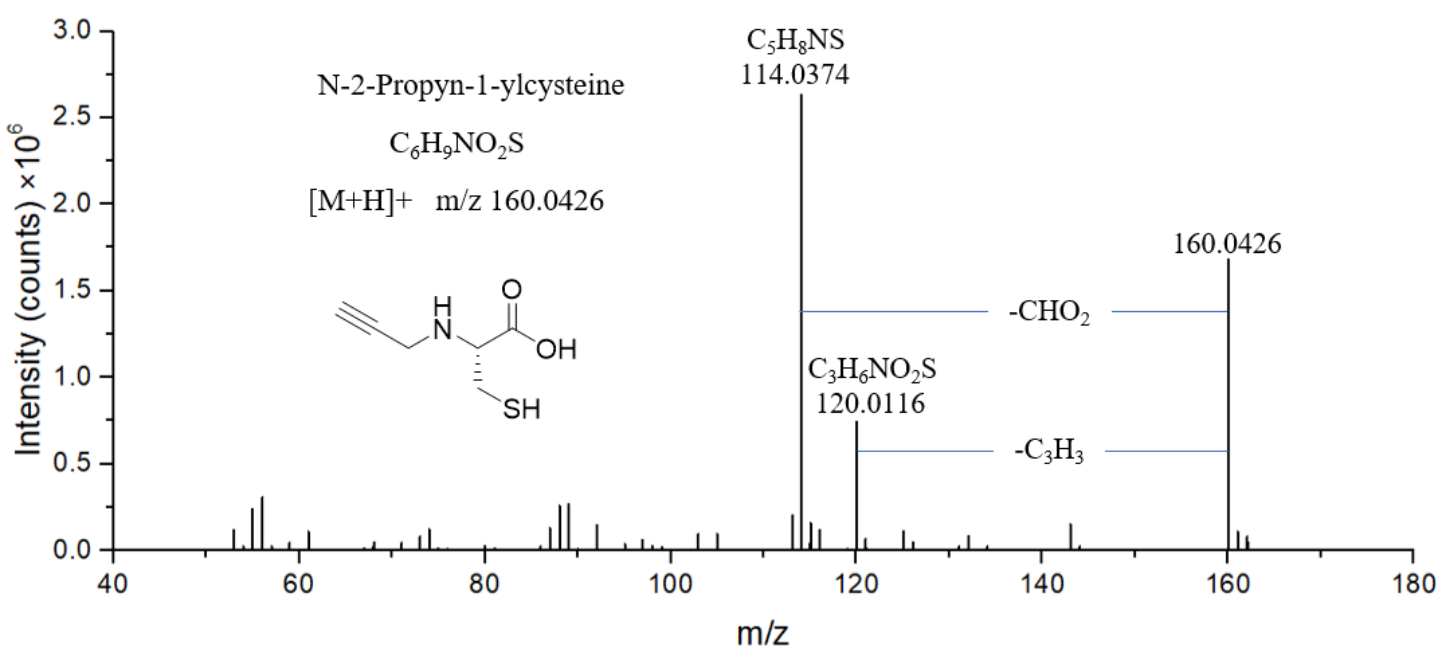

No. 12 Cycloalliin

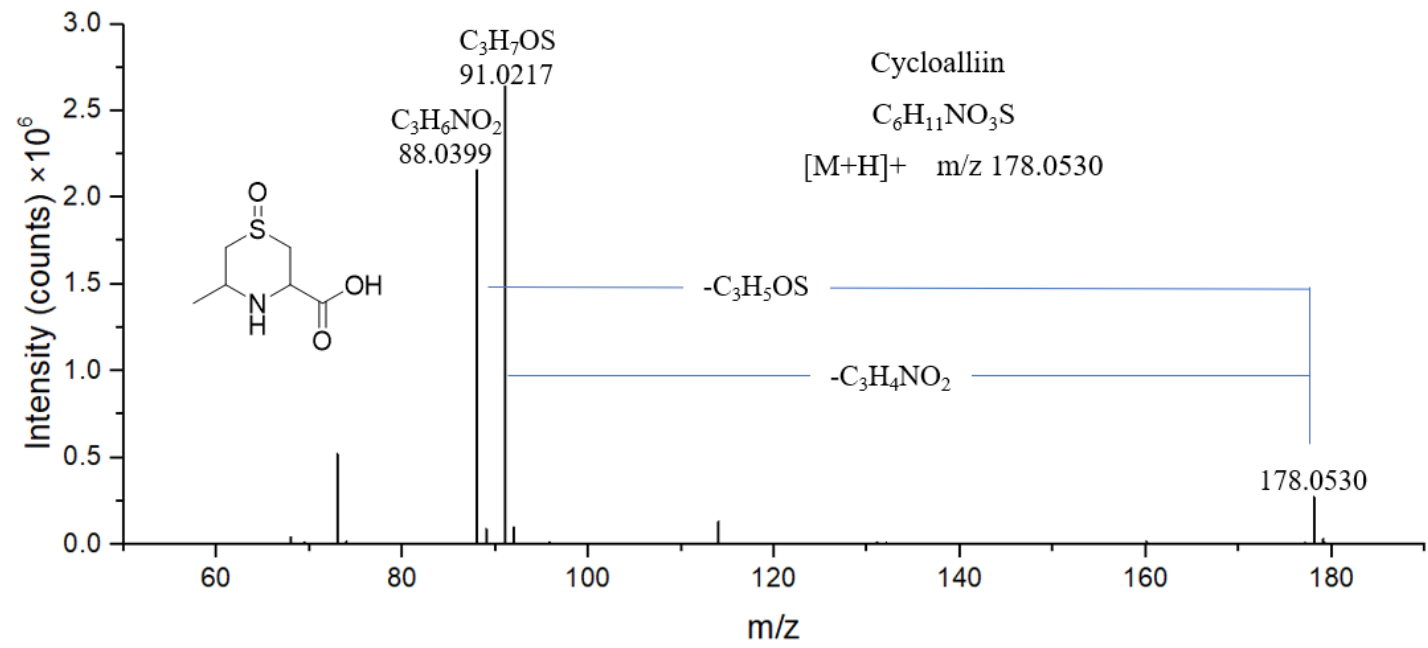

No. 13 S-Propyl-L-cysteine sulfoxide

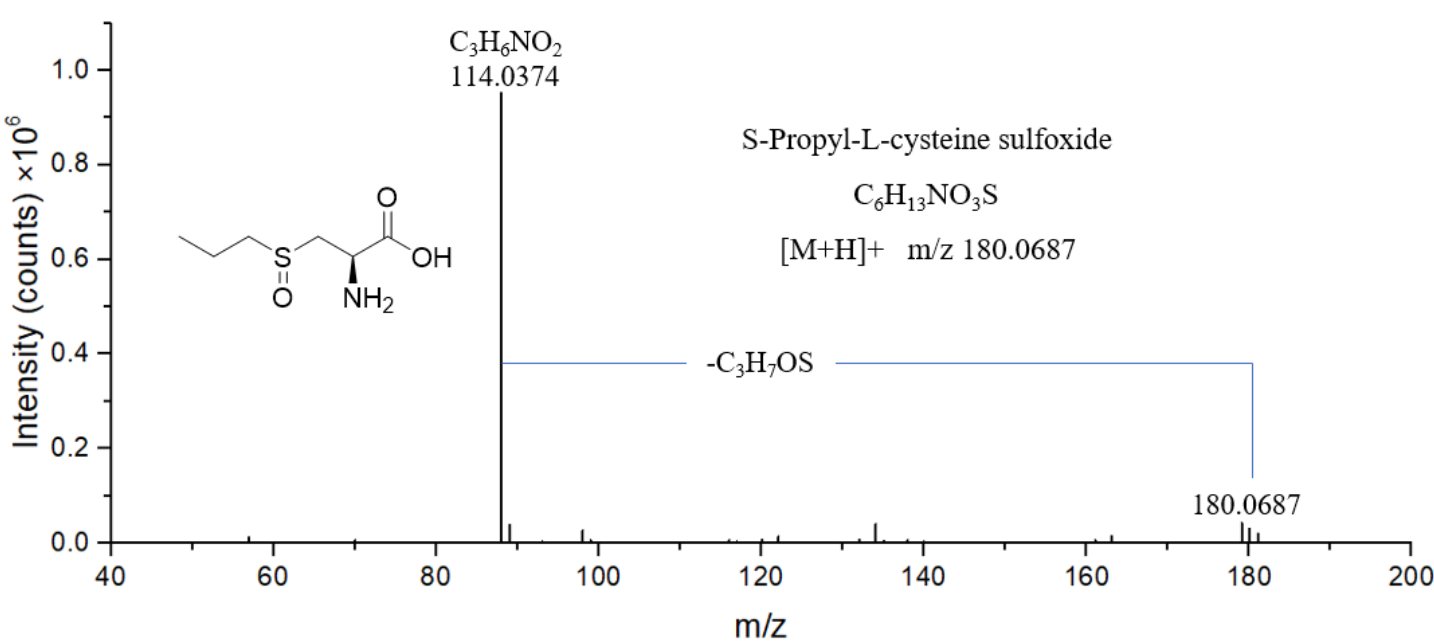


No. 40 S-(2-Carboxypropyl)cysteine

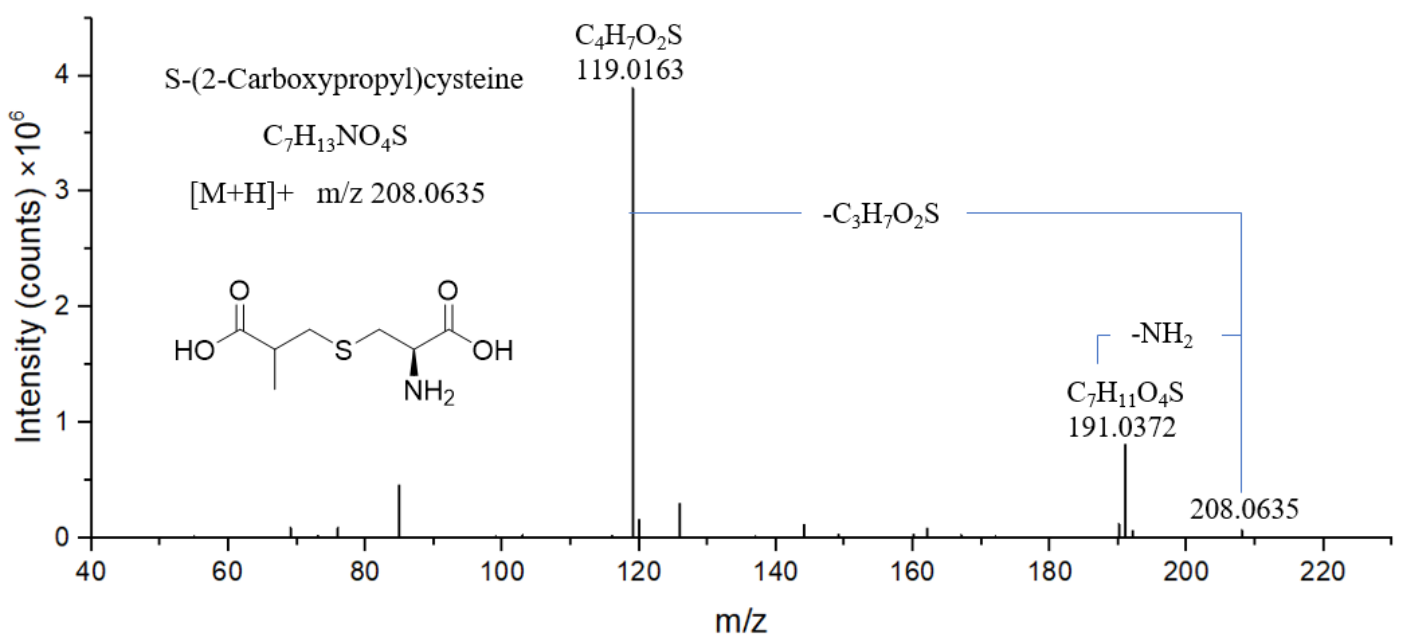

No. 55 N(6)-Methyllysine

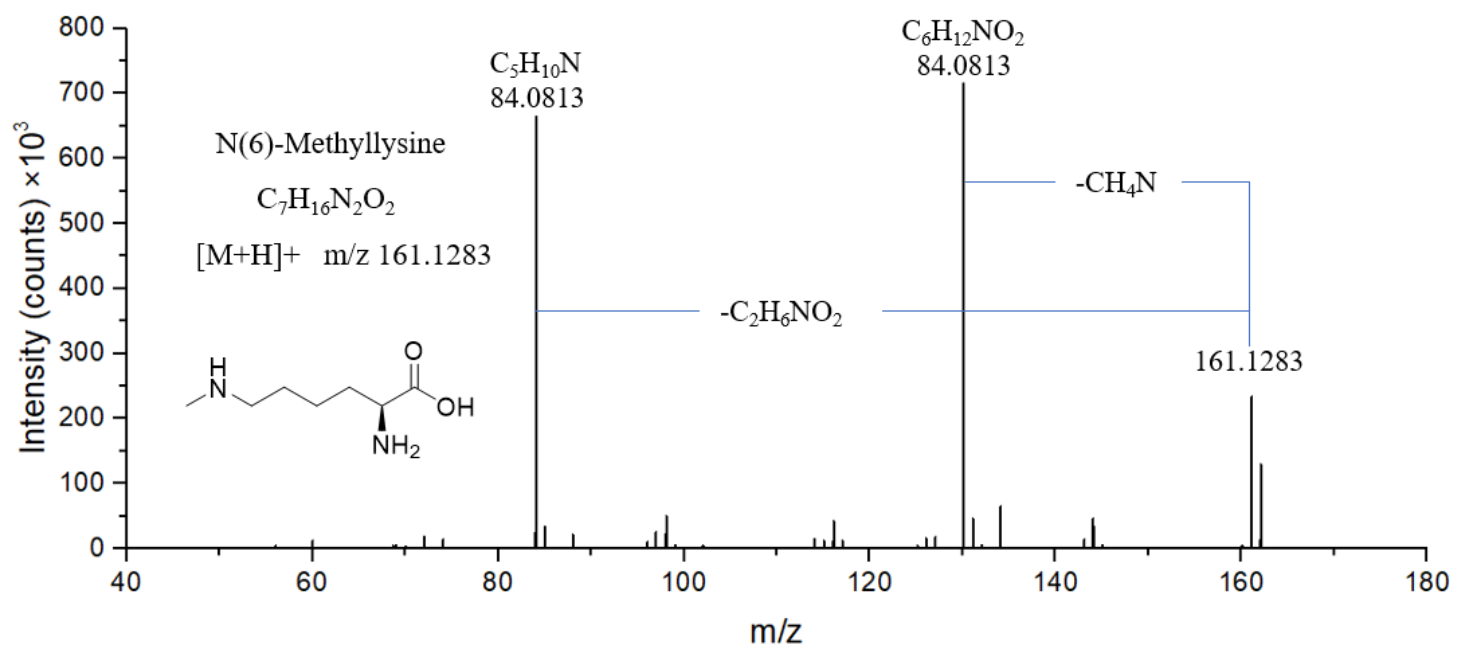

No. 56 N(6),N(6)-Dimethyl-L-lysine

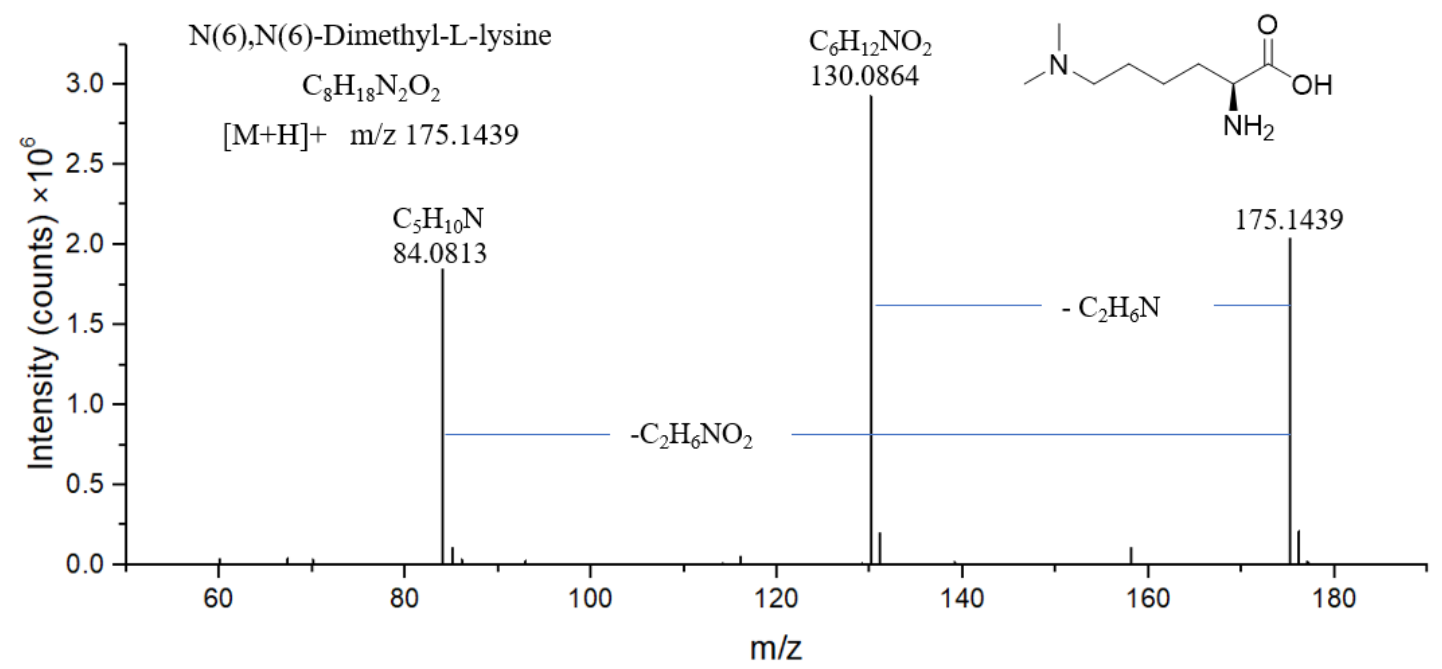


No. 57 N-Methyl-L-methionine

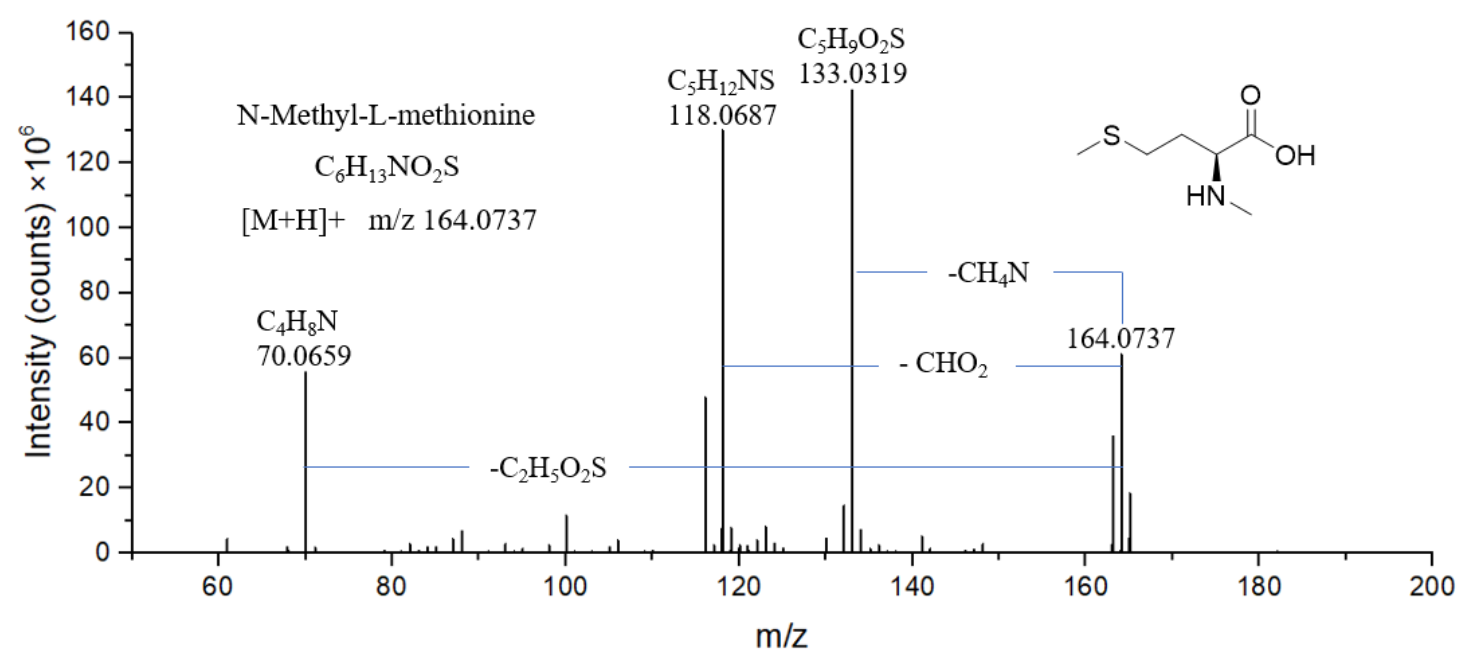

No. $58 \delta$-Guanidinovaleric acid

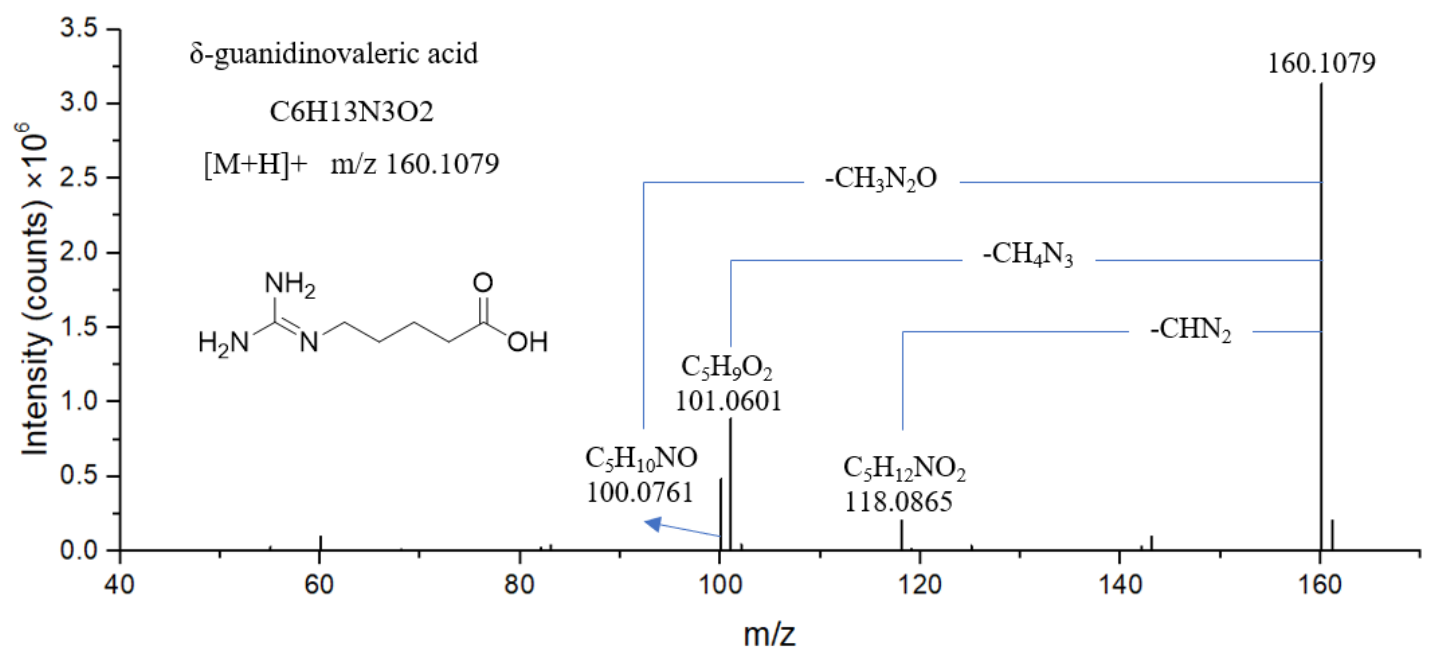

No. 78 Choline sulfate

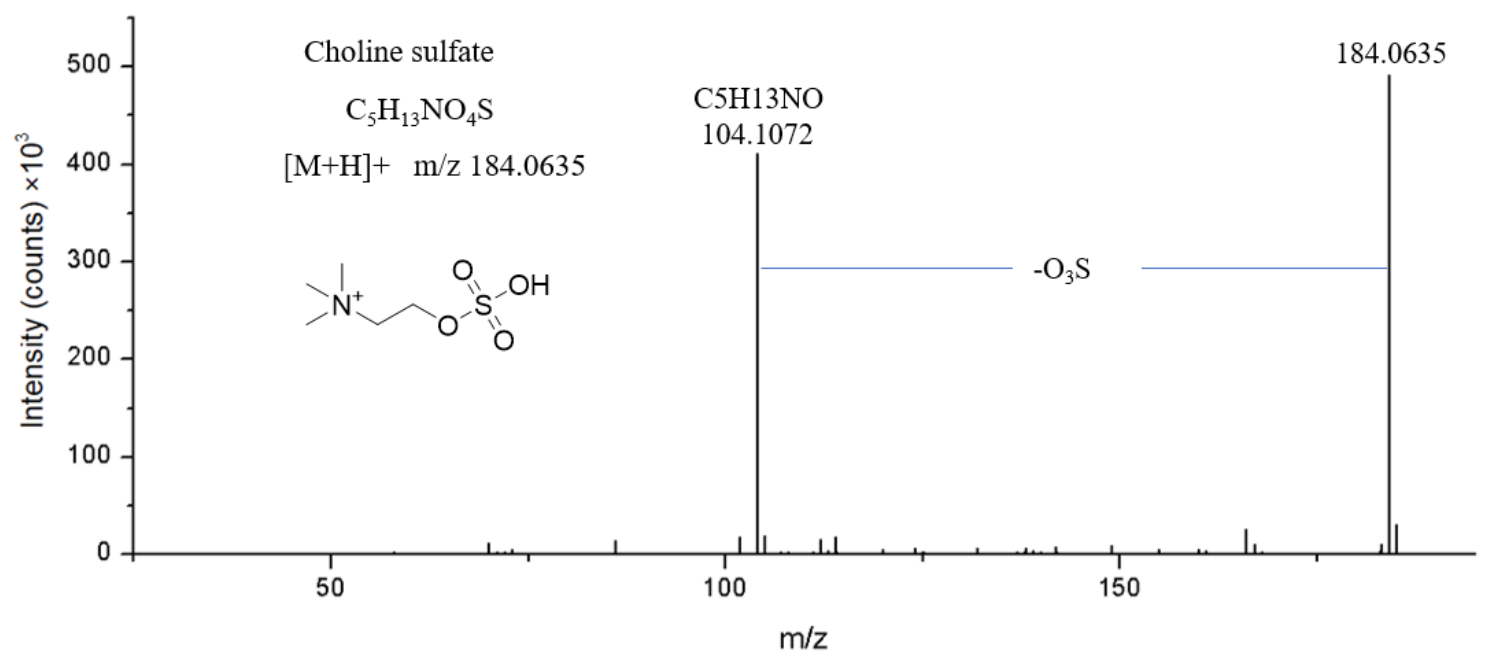


No. 90 4-Guanidinobutanal

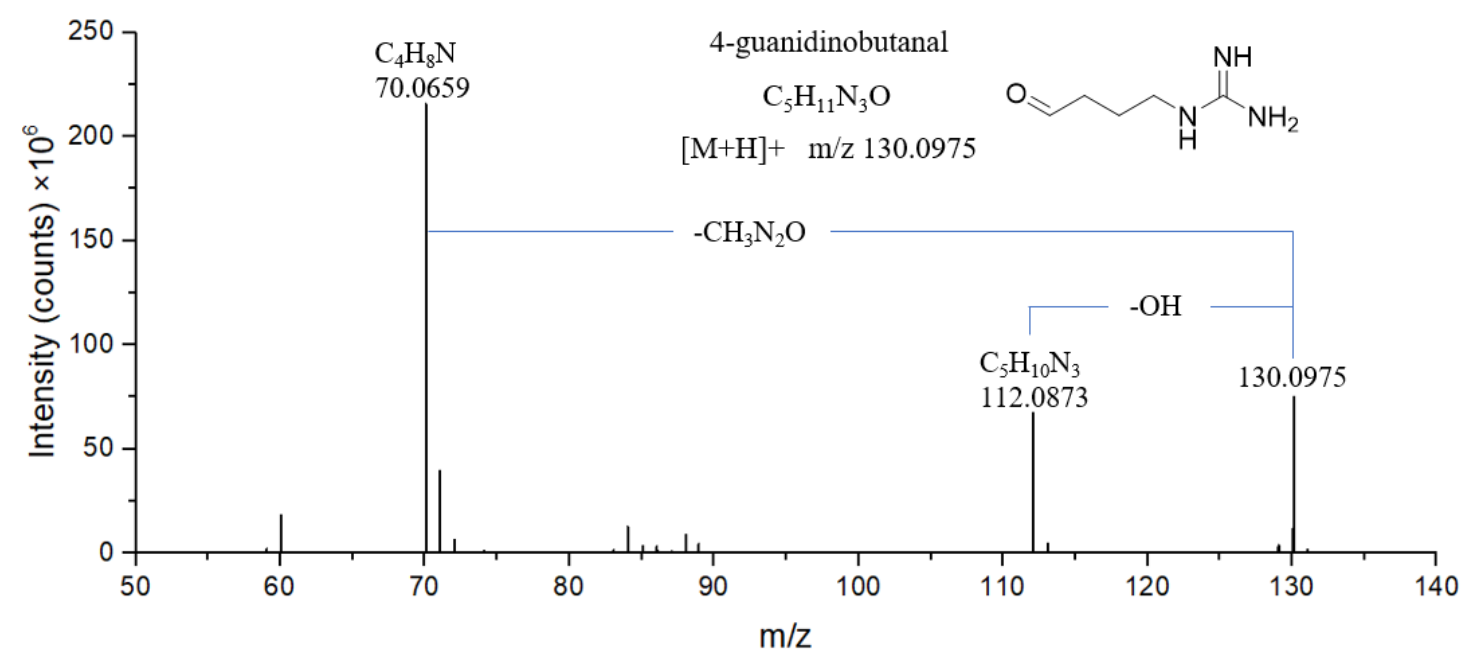

Figure S3 Structural characterization for 13 compounds in garlic
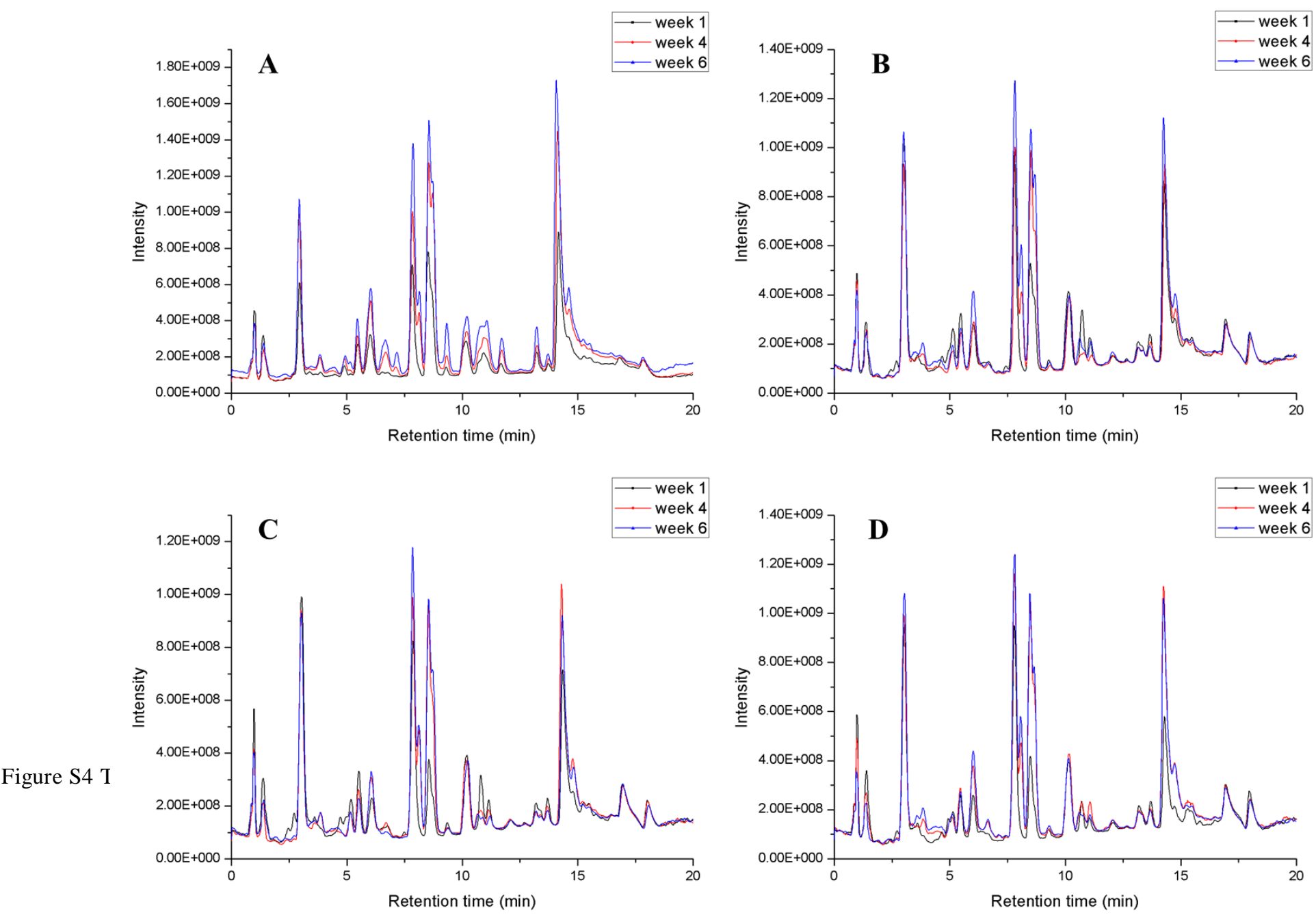


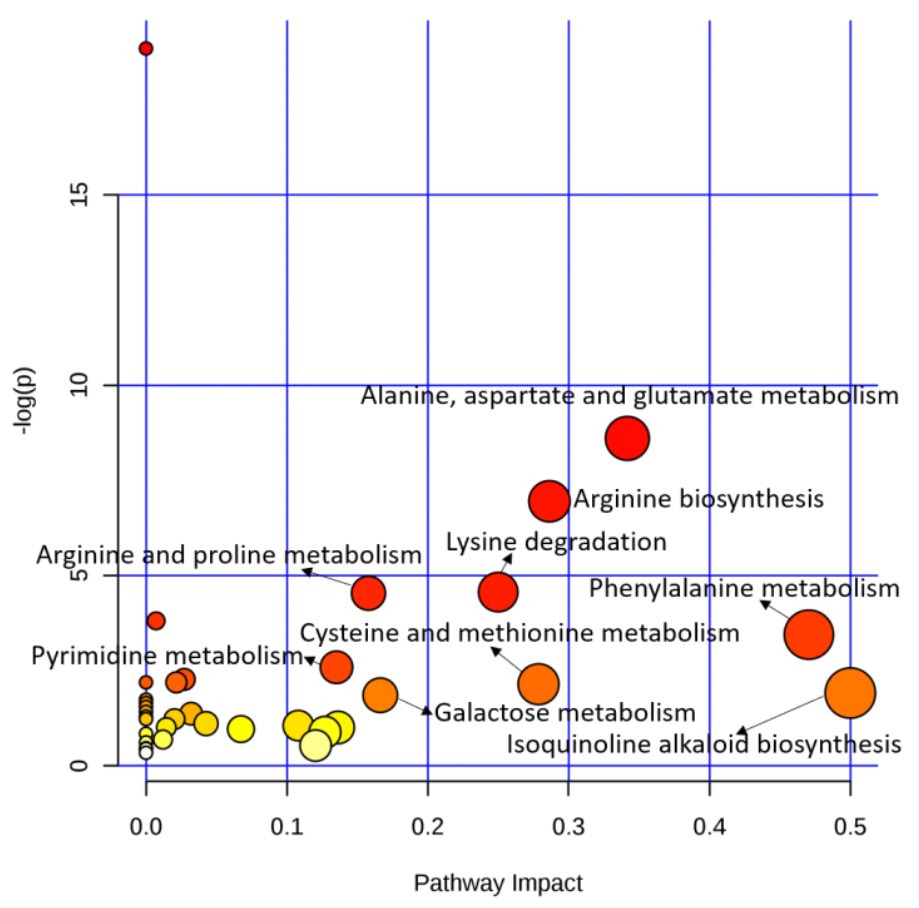

Figure S5 Pathway analysis showing changing metabolism during garlic development. The size and color of the circle represented the pathway impact and pathway significance, respectively. The bigger and deeper circles at the upper-right corner were the pathways that had a strong impact with higher significance in garlic metabolism. 